\title{
Habitable-Zone Exoplanet Observatory baseline 4-m telescope: systems-engineering design process and predicted structural thermal optical performance
}

\author{
H. Philip Stahl,, ${ }^{\mathrm{a}, *}$ Gary Kuan, ${ }^{\mathrm{b}}$ William R. Arnold, ${ }^{\mathrm{c}}$ Thomas Brooks, ${ }^{\mathrm{a}}$ \\ J. Brent Knight, ${ }^{\mathrm{a}}$ and Stefan Martin ${ }^{\mathrm{b}}$ \\ ${ }^{a}$ NASA Marshall Space Flight Center, Huntsville, Alabama, United States \\ bJet Propulsion Laboratory, Pasadena, California, United States \\ ${ }^{c}$ a.i. Solutions, Huntsville, Alabama, United States
}

\begin{abstract}
The Habitable-Zone Exoplanet Observatory Mission (HabEx) is one of four large missions under review for the 2020 astrophysics decadal survey. Its goal is to directly image and spectroscopically characterize planetary systems in the habitable zone around nearby Sun-like stars. In addition, HabEx will perform a broad range of general astrophysics science enabled by a 115- to $1700-\mathrm{nm}$ spectral range and $3 \times 3$ arcminute field of view. Critical to achieving its science goals, HabEx requires a large, ultrastable UV/optical/near-IR telescope. Using sciencedriven systems engineering, HabEx specified its baseline telescope to be a 4-m off-axis, unobscured three-mirror anastigmatic architecture with diffraction-limited performance at $400 \mathrm{~nm}$, and wavefront stability on the order of a few tens of picometers. We summarize the systems-engineering approach to the baseline telescope assembly's optomechanical design, including a discussion of how science requirements drive the telescope's specifications. We also present structural thermal optical performance analysis showing that the baseline telescope structure meets its specified tolerances. We report new and updated analysis that is not in the HabEx final report. () The Authors. Published by SPIE under a Creative Commons Attribution 4.0 Unported License. Distribution or reproduction of this work in whole or in part requires full attribution of the original publication, including its DOI. [DOI: 10.1117/1.JATIS.6.3.034004]
\end{abstract}

Keywords: space telescopes; telescope design; STOP modeling; exoplanet; astrophysics; HabEx.

Paper 20034 received Apr. 7, 2020; accepted for publication Jul. 31, 2020; published online Aug. 17, 2020.

\section{Introduction}

"Are we alone in the Universe?" is maybe the most compelling science question of our generation. Per the 2010 New Worlds, New Horizons decadal survey: "One of the fastest growing and most exciting fields in astrophysics is the study of planets beyond our solar system. The goal is to image rocky planets that lie in the habitable zone of nearby stars." ${ }^{.1}$ The survey recommended, as its highest priority, medium-scale activity such as a "New Worlds Technology Development Program" to "lay the technical and scientific foundations for a future space imaging and spectroscopy mission." The National Research Council report, NASA Space Technology Roadmaps $\&$ Priorities, ${ }^{2}$ states that the second-highest technical challenge for the National Aeronautics and Space Administration (NASA) for expanding our understanding of Earth and the universe in which we live is to "develop a new generation of astronomical telescopes that enable discovery of habitable planets, facilitate advances in solar physics, and enable the study of faint structures around bright objects by developing high-contrast imaging and spectroscopic technologies to provide unprecedented sensitivity, field of view (FOV), and spectroscopy of faint objects."

In preparation for the 2020 decadal survey, NASA performed detailed concept studies of four potential missions. One of these was the Habitable-Zone Exoplanet Observatory (HabEx) Mission. HabEx has three goals: to seek out nearby worlds and explore their habitability, to map out nearby planetary systems and understand the diversity of the worlds they contain, and

*Address all correspondence to H. Philip Stahl, E-mail: h.philip.stahl@ nasa.gov 
to carry out observations that open new windows on the universe from the ultraviolet through near-IR (UV-NIR). The HabEx Science and Technology Definition Team selected as 'Architecture A' a 4-m telescope with four science instruments (coronagraph, Starshade instrument, UV-NIR imaging multiobject slit spectrograph, and high-resolution UV spectrograph), and a 52-m external starshade occulter. ${ }^{3}$

Section 2 reviews how the HabEx optical telescope assembly (OTA) specifications derive from the HabEx science requirements. Section 3 describes the baseline optomechanical OTA design, key components, and features. Section 4 summarizes detailed performance analysis of the baseline optomechanical design, which shows that the design, using proven technology and engineering practices, can achieve the specifications necessary to perform HabEx science. Section 5 shows how structural thermal optical performance (STOP) model predictions are used to construct an error budget. Section 6 presents an analysis of the telescope's response to an impulse. The baseline 4-m, off-axis HabEx optomechanical telescope design "closes" for its line of sight (LOS) and wavefront error (WFE) stability specifications. The only external assumptions are that the mission is launched in a Space Launch System (SLS) 8.4-m fairing, and the spacecraft uses low-disturbance microthrusters for pointing control.

\section{OTA Specifications}

The HabEx OTA design team followed a science-driven, systems-engineering method. Design element specifications were derived from science requirements (Table 1), and error budgets were created for each specification.

Exoplanet science drives the choice of an off-axis architecture, aperture diameter, and primary mirror $(\mathrm{PM}) F / \#$. General astrophysics and its desire for a $3 \times 3$ arcminute FOV drives the choice of a three-mirror anastigmatic (TMA) optical design (Fig. 1). UV science drives the spectral range and operating temperature. Both exoplanet and general astrophysics sciences need 400-nm diffraction-limited telescope performance. Diffraction limit, LOS jitter, and WFE stability are discussed in Secs. 2.1-2.3.

Table 1 HabEx optical telescope specification flow-down from science requirements.

\begin{tabular}{|c|c|c|}
\hline Science & Telescope & Specification \\
\hline \multirow{7}{*}{$\begin{array}{l}\text { Exoplanet: inner working } \\
\text { angle }\end{array}$} & Architecture & Off-axis unobscured circular aperture \\
\hline & Aperture diameter & $>3.7 \mathrm{~m}$ \\
\hline & Primary mirror $F / \#$ & $\begin{array}{l}\text { Slower than } F / 2.25 \text { to minimize polarization } \\
\text { crosstalk }\end{array}$ \\
\hline & Diffraction limit & $400 \mathrm{~nm}$ \\
\hline & WFE stability & $\begin{array}{l}1 \text { to } 250 \mathrm{pm} \text { depending on coronagraph and } \\
\text { spatial frequency }\end{array}$ \\
\hline & LOS stability (jitter) & $<0.3$ mas per axis \\
\hline & Science instruments & $\begin{array}{l}\text { On the side (in the SM tower structure) to } \\
\text { maximize thermal stability and to minimize } \\
\text { reflecting angles for polarization. }\end{array}$ \\
\hline \multirow[t]{2}{*}{$\begin{array}{l}\text { General astrophysics: } \\
\text { wide FOV }\end{array}$} & Three-mirror anastigmatic & $\begin{array}{l}3 \times 3 \text { arcmin per instrument inside } 21 \text { arcmin } \\
\text { diameter FOV }\end{array}$ \\
\hline & Diffraction limit & $400 \mathrm{~nm}$ \\
\hline \multirow[t]{2}{*}{ UV science } & Spectral range & 115 to $1700 \mathrm{~nm}$ (nominal) \\
\hline & Operating temperature & $270 \mathrm{~K}$ (nominal); $260 \mathrm{~K}$ (minimum) \\
\hline
\end{tabular}




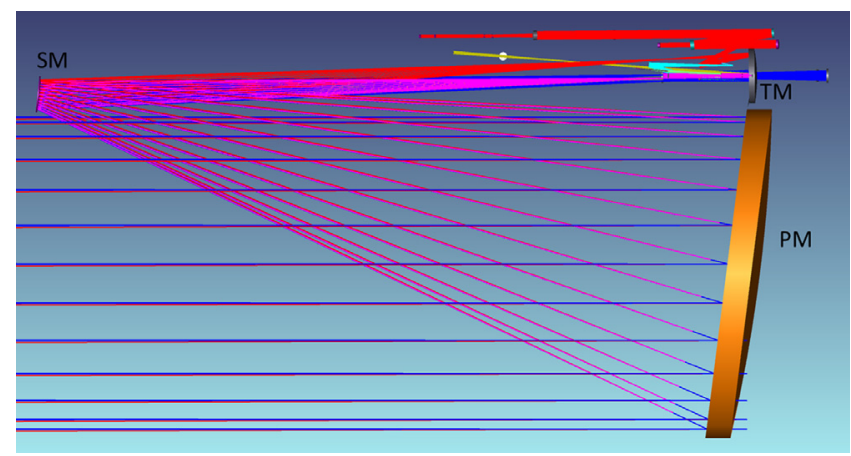

Fig. 1 TMA optical design.

Imaging habitable-zone exoplanets using a coronagraph requires a telescope/coronagraph system that can produce a $10^{-10}$ contrast "dark hole" with the smallest inner working angle (IWA) possible and as large of an irradiance throughput as possible. IWA is the minimum angular distance (on the sky) where the dark hole begins - the location where the coronagraph can block $10^{10}$ of the host star's brightness (Fig. 2). The number of habitable zones that can be searched will be greater when the IWA is smaller and the throughput is larger. The ability to achieve a small IWA depends on the telescope's ability to produce a small stable point spread function (PSF) with a compact stable encircled energy (EE). Therefore, when the EE is smaller, the IWA is smaller. It is common knowledge that when the PSF and EE are smaller, the telescope's aperture will be larger. However, what is often overlooked is that an unobscured (off-axis) telescope always has a more compact EE (better IWA) than an on-axis telescope with a central obscuration, because diffraction from the central obscuration broadens the PSF. To be specific, an unobscured circular aperture has $82.8 \% \mathrm{EE}$ at $\lambda / D$, and a telescope with a $10 \%$ central obscuration has $82.5 \% \mathrm{EE}$ at $1.4 \lambda / D$ (and for a $20 \%$ obscuration, $82 \% \mathrm{EE}$ is at $1.63 \lambda / D$ ). ${ }^{4}$ Thus, to achieve the same IWA performance as an unobscured 4-m telescope, an on-axis telescope with $10 \%$ central obscuration would need to be at least $5.6 \mathrm{~m}$, and one with $20 \%$ obscuration would need to be at least $6.5 \mathrm{~m}$. In addition, diffraction from secondary mirror (SM) spider obscurations distorts the PSF and broadens the EE. A 1-2\% wide spider can increase EE diameter IWA by $5-10 \%$, requiring a $5-10 \%$ larger on-axis telescope. ${ }^{4}$ Of course, the problem is even worse for a segmented aperture PM.

A key attribute of a coronagraph is its core throughput. Core throughput is the fraction of the planet light collected by the telescope PM that ends up inside the core region. Figure 3 shows the core throughput for three coronagraphs (vector-vortex coronagraph charge 4 (VVC-4),

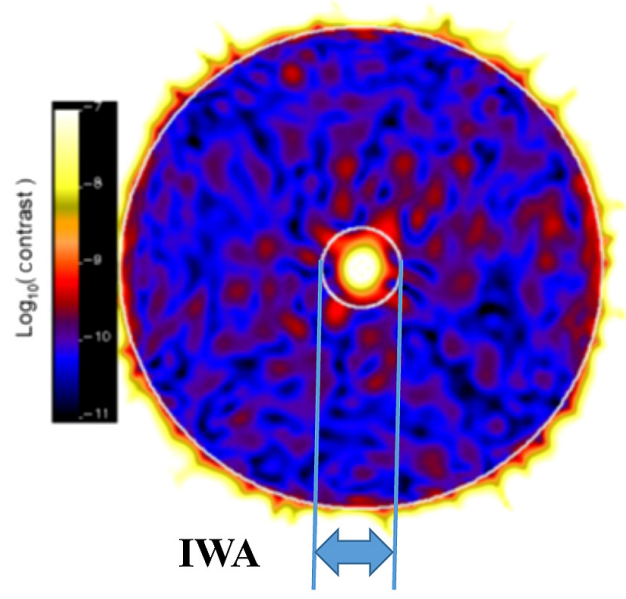

Mean dark hole contrast $=1.0 \times 10^{-10}$

Fig. 2 Dark hole. 


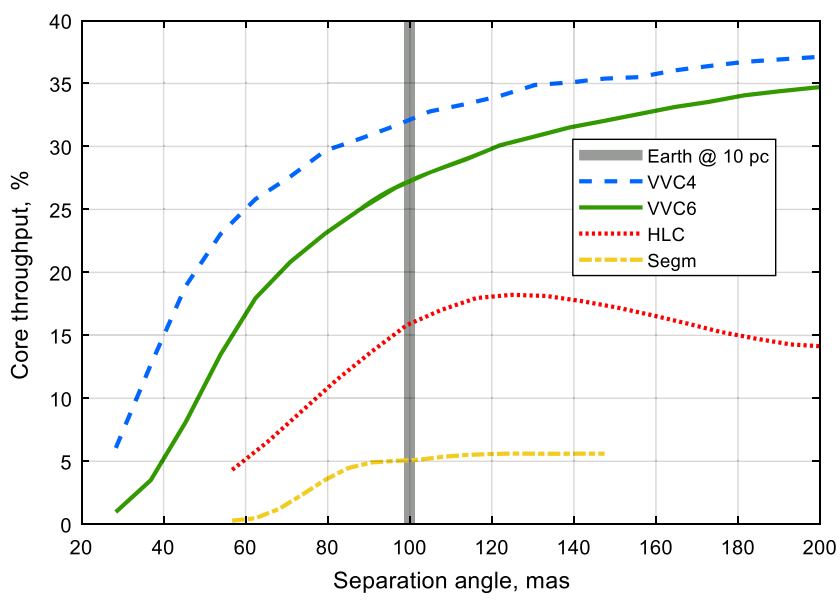

Fig. 3 Core throughput versus separation angle for three coronagraphs [vector-vortex coronagraph charge 4 (VVC-4), charge 6 (VVC-6), and hybrid Lyot (HLC)] with the HabEx baseline 4-m, off-axis, unobscured telescope, and 6-m, on-axis, segmented PM telescope (Segm) with an APLC. Vertical line is Exo-Earth at 10 pc (100 mas) $)^{5,6}$

charge 6 (VVC-6), and hybrid Lyot (HLC) with the HabEx baseline 4-m, off-axis, unobscured telescope) and the throughput for a 6-m, on-axis, segmented PM telescope (Segm) (e.g., the James Webb Space Telescope, JWST) with an apodized pupil Lyot coronagraph (APLC). ${ }^{5,6}$

Regarding minimum aperture and diffraction limit, the specification is based on a design reference mission (DRM) yield estimate for an off-axis-telescope/coronagraph combination. ${ }^{7}$ Threshold science occurs when the telescope PSF core radius $(\lambda / D)$ is $<25$ mas. This is accomplished with a $>3.7-\mathrm{m}$ off-axis monolithic telescope with a 400-nm diffraction limit.

Regarding the PM's $F / \#$, to minimize package size, a fast PM $F / \#$, or short radius of curvature is desired. But to minimize polarization cross-talk in the coronagraph, a slow PM $F / \#$ is required. An optical design similar to Exo-C was selected, with an $F / 2.5 \mathrm{PM}$ and the science instruments located on the anti-Sun side of the telescope. ${ }^{8}$ This architecture provides the instruments with a thermally stable environment. Additional benefits of this configuration are that it minimizes the need for high angle of incidence reflections that produce unwanted polarization effects and isolates the coronagraph from thermal disturbances. Consequently, the OTA is physically long. The baseline 4-m design has a PM-to-SM separation of $\sim 9 \mathrm{~m}$.

\subsection{Diffraction-Limited Performance}

Diffraction limit is driven by both general astrophysics and coronagraphy because, along with aperture diameter, diffraction limit directly relates to the performance parameters of PSF size, EE, and Strehl ratio. Applying the Marechal approximation, to have a 400-nm diffraction-limited telescope requires a static system WFE of $\sim 30 \mathrm{~nm}$ rms. Contributors to the telescope WFE are the PM, SM, and tertiary mirror (TM) surface figure errors (SFE), as well as the ability to attach the PM and SM to the structure and accurately align them to the TM, while maintaining that alignment on-orbit (Fig. 4). Because the telescope has a laser metrology system that establishes and maintains PM and SM alignment to the TM with an uncertainty of better than $7 \mathrm{~nm} \mathrm{rms}$, the majority of the telescope's WFE budget can be allocated to the mirrors. Moreover,

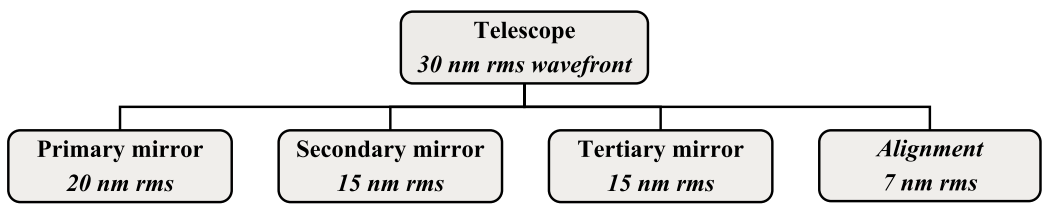

Fig. 4 Simplified system WFE budget allocation flowdown. 


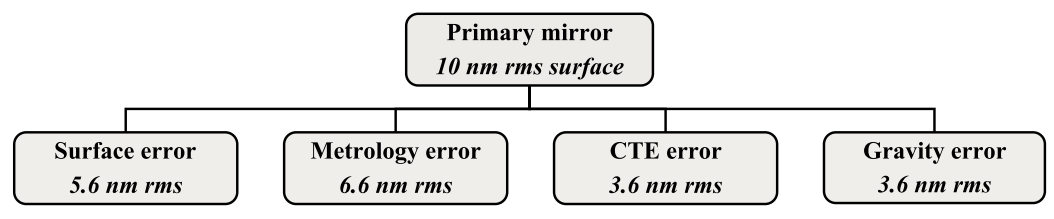

Fig. 5 PM specification allocation.

because the PM is larger and less stiff than the SM or TM, it gets the largest allocation. Note that the measured Wide Field Infrared Survey Telescope (WFIRST) PM WFE is $13.2 \mathrm{~nm} \mathrm{rms}$.

Figure 5 shows how the PM WFE allocation flows into nominal PM engineering specifications. Recall that surface error is half of WFE and that these specifications are independent of aperture size. This error budget defines the engineering specifications that must be achieved by the PM fabrication process. The SFE allocation is the allowed fabrication error between the specified surface and the as-built surface. It is set to what was demonstrated on WFIRST. ${ }^{9}$ Metrology error is the uncertainty to which the SFE is measured and is set to what was demonstrated on JWST. ${ }^{10}$ The last two boxes define specific PM error contributors: uncertainty in the ability to quantify and remove coefficient of thermal expansion (CTE) homogeneity effects and uncertainty in the ability to quantify and remove gravity sag from the PM surface shape.

\subsubsection{PM SFE}

General astrophysics and exoplanet science drive the PM surface specification as a function of spatial frequency. General astrophysics is most sensitive to the shape and stability of the PSF driven by low-spatial frequency errors. Exoplanet science high-contrast imaging is most sensitive to mid- and high-spatial frequency errors. Mid-spatial frequency errors blur or spread the core. High-spatial frequency errors and surface roughness scatter light out of the core and over the entire PSF. Thus, per Table 2, the total PM surface figure specification is further divided into low-, mid-, and high-spatial frequency bands.

The specification of Table 2 assumes computer-controlled polishing for spatial frequencies of up to 30 cycles (50 mm minimum tool size) to correct quilting error with a power spectral density (PSD) slope of -2.5 for high-spatial frequencies. The 100-cycle boundary between mid- and high-spatial errors assumes that the coronagraph uses a $64 \times 64$ deformable mirror (DM). A $64 \times 64 \mathrm{DM}$ can theoretically correct spatial frequencies up to 32 cycles (or half the number of DM elements). This could create a dark hole with an IWA of $\lambda / D$ and an outer working angle (OWA) of $32 \lambda / D$. The systems-engineering consideration is that PM spatial frequency errors up to $3 \times$ beyond what can be corrected by the DM can scatter energy back into the dark hole. Therefore, the PM needs to have the smoothest surface figure possible for spatial frequency errors from 30 to 100 cycles. $^{11,12}$

Finally, note that if the PM is segmented, then the low-spatial SFE must be subdivided between segment SFE $(N-30$ cycles/diameter) and segment-to-segment phasing $(<N$ cycles/diameter where $N=$ number of segments). A $50 / 50$ division would be $2.5 \mathrm{~nm} \mathrm{rms}$ for each.

Table 2 HabEx PM SFE specification.

\begin{tabular}{lcc}
\hline \hline & Surface & Wavefront \\
\cline { 2 - 3 } Total error & $<5.6 \mathrm{~nm} \mathrm{rms}$ & $<11.2 \mathrm{~nm} \mathrm{rms}$ \\
\hline Low spatial SFE (<30 cycles/diameter) & $<4.3 \mathrm{~nm} \mathrm{rms}$ & $<8.6 \mathrm{~nm} \mathrm{rms}$ \\
Mid-spatial SFE (30 to 100 cycles/diameter) & $<3.3 \mathrm{~nm} \mathrm{rms}$ & $<6.6 \mathrm{~nm} \mathrm{rms}$ \\
High spatial SFE ( $>100$ cycles/diameter) & $<1.4 \mathrm{~nm} \mathrm{rms}$ & $<2.8 \mathrm{~nm} \mathrm{rms}$ \\
Roughness & $<0.3 \mathrm{~nm} \mathrm{rms}$ & $<0.6 \mathrm{~nm} \mathrm{rms}$ \\
\hline \hline
\end{tabular}




\subsubsection{PM CTE error}

CTE errors can introduce low- and mid-spatial frequency SFE. CTE errors have two components. First is knowledge of the exact temperature at which the mirror's CTE is zero. Any operational departure from this temperature will introduce a WFE_-primarily power. Fortunately, this error can be mitigated by adjusting the on-orbit operating temperature for a minimum WFE. The second, and more important, error is mid-spatial frequency residual cryo-null-polishing error. Because of CTE inhomogeneity in the mirror substrate, the mirror will have a different shape at its operating temperature than at its manufacture temperature. This is a problem for cryogenic telescopes, which can be mitigated via cryo-null-figuring (CNF). For example, the JWST mirror segments, which operate at $30 \mathrm{~K}$ ( $260 \mathrm{~K}$ below their manufacture temperature), had to undergo CNF for many tens of nanometers of cryodeformation. ${ }^{10} \mathrm{HabEx}$ requires CNF because it has an extremely smooth SFE specification and it plans to operate at a nominal temperature of $270 \mathrm{~K}$ (i.e., $30 \mathrm{~K}$ below its likely fabrication temperature). The exact amount of WFE that must undergo CNF depends on the mirror substrate's CTE homogeneity. For example, Fig. 6 shows a 1.2-m extreme-lightweight ZERODUR ${ }^{\circledR}$ mirror (ELZM) with a 9.8-nm rms cryodeformation over a 62-K temperature range. Analysis indicates that this mirror has a CTE homogeneity of $\pm 5 \mathrm{ppb} / \mathrm{K}$ (parts per billion/K), and its surface changes by $0.18 \mathrm{~nm} / \mathrm{K}^{13}$ Thus, the maximum CTE error shift from its $300-\mathrm{K}$ manufacture temperature to its $270-\mathrm{K}$ operating temperature is the error budget allocation of $3.6 \mathrm{~nm}$ rms. Obviously, this error can be mitigated by CNF or DM correction.

\subsubsection{PM gravity sag figure error}

A gravity sag error is primarily low-spatial frequency. It occurs because, during fabrication, mirrors experience self-weight deflection when attached to their metrology mount. However, in the $0-\mathrm{G}$ environment of space, there is no self-weight deflection. The change in the mirror's shape from 1 to 0 (zero) $\mathrm{G}$ is called G-release. To produce a diffraction limited space telescope, the PM must be fabricated to its required on-orbit $0-\mathrm{G}$ figure. This is accomplished by quantifying and removing gravity sag from the mirror fabrication process. Any uncertainty in this removal is called G-release error. For HabEx, the desired G-release error is $<4 \mathrm{~nm} \mathrm{rms.}$

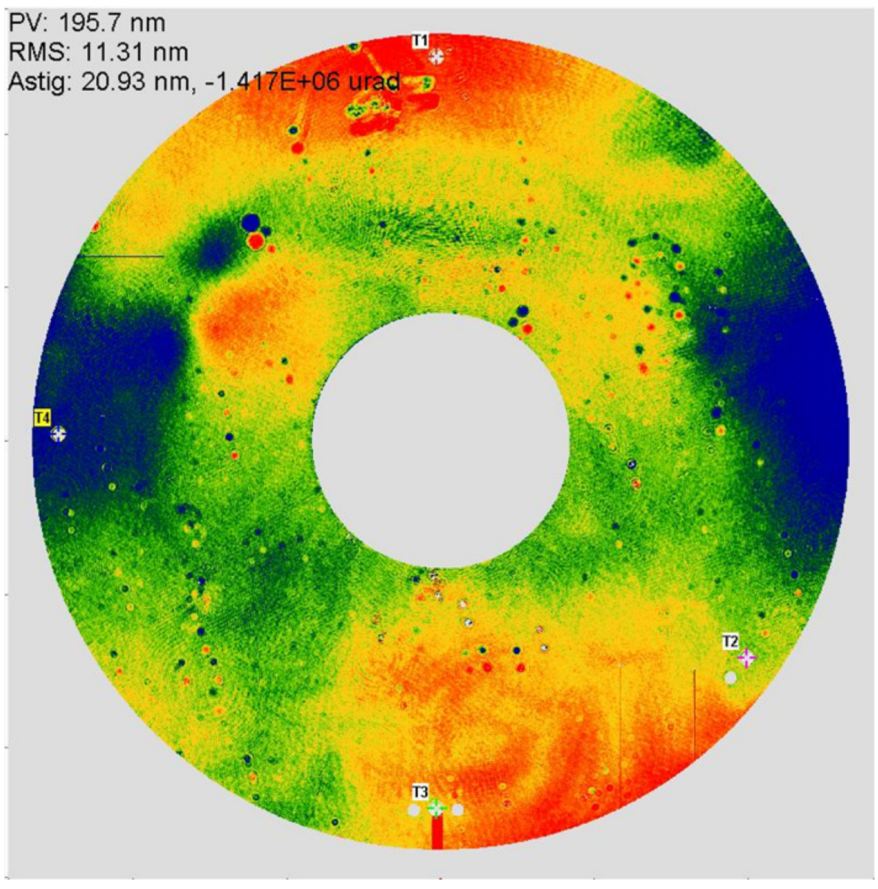

\begin{tabular}{|c|c|l|c|}
\hline \multicolumn{4}{|c|}{ Measured delta-SFE 292-230K } \\
\hline Index & \multicolumn{2}{|c|}{ Total RMS } & $\mathbf{9 . 7 6}$ \\
\hline N & M & \multicolumn{1}{|c|}{ Zernike term } & nm rms \\
\hline 1 & 1 & Tilt & 0.05 \\
\hline 2 & 0 & Power & 0.24 \\
\hline 2 & 2 & Astig & 8.55 \\
\hline 3 & 1 & Coma & 0.90 \\
\hline 4 & 0 & Spherical & 0.27 \\
\hline 3 & 3 & Trefoil & 2.15 \\
\hline 4 & 2 & Secondary astig & 0.72 \\
\hline 5 & 1 & Secondary coma & 0.75 \\
\hline 6 & 0 & Secondary spherical & 0.40 \\
\hline 4 & 4 & Tetrafoil & 1.73 \\
\hline 5 & 3 & Secondary trefoil & 1.39 \\
\hline 6 & 2 & Tertiary astig & 0.93 \\
\hline 7 & 1 & Tertiary coma & 0.77 \\
\hline 8 & 0 & Tertiary spherical & 0.24 \\
\hline 5 & 5 & Pentafoil & 0.53 \\
\hline 6 & 4 & Secondary tetrafoil & 0.29 \\
\hline 7 & 3 & Tertiary trefoil & 1.72 \\
\hline 8 & 2 & Quaternary astig & 0.04 \\
\hline 9 & 1 & Quaternary coma & 0.81 \\
\hline 10 & 0 & Quaternary spherical & 0.57 \\
\hline 6 & 6 & Hexafoil & 1.28 \\
\hline 7 & 5 & Secondary pentafoil & 0.49 \\
\hline 8 & 4 & Tertiary tetrafoil & 0.48 \\
\hline 9 & 3 & Quaternary trefoil & 1.20 \\
\hline 10 & 2 & Quinary astig & 0.58 \\
\hline 11 & 1 & Quinary coma & 0.45 \\
\hline 12 & 0 & Quinary spherical & 0.73 \\
\hline & & \\
\hline
\end{tabular}

Fig. 6 1.2-m Schott ELZM $62 \mathrm{~K}$ thermal deformation. 
Of course, this error could be corrected via a DM, but it is better for the PM to have a good $0-\mathrm{G}$ figure.

Gravity sag consists of two components: self-weight mount deflection and facesheet deflection. Self-weight mount deflection occurs due to the reaction or bending of the mirror gravity against its optomechanical support mount in a gravitational field. Facesheet deflection occurs because space mirrors are typically light-weight-leaving parts of the facesheet without support. These unsupported parts deflect due to the acceleration of gravity against the vertical core ribs. For the purposes of this paper, we will ignore facesheet gravity deflection because it is typically a smaller effect than fabrication quilting. The reason is that force exerted onto the facesheet by grinding and polishing is typically greater than that of gravity. Also, methods to mitigate facesheet deflection and quilting at the scale of HabEx's 4-m mirror are Technology Readiness Level 9 (TRL-9). One proven approach for mitigating this issue is to optimize the mirror's facesheet thickness and core pocket dimensions. Another approach is to pressurize the mirror core cells to balance gravity during the fabrication process.

\subsection{LOS Stability Specification}

LOS instability is important for both general astrophysics and coronagraphy because it causes PSF smearing that degrades spatial resolution and IWA. A typical specification for LOS error is $<1 / 10$ th the PSF radius. For a 400-nm-diffraction-limited 4-m telescope, the on-sky PSF radius is $\sim 20$ mas. Thus, the specification should be $<2$ mas. But coronagraphs require LOS stability better than 0.3 mas per axis. This is because LOS error causes beam-shear on the SM and TM, as well as other mirrors in the optical train, which introduces dynamic WFE instability resulting in contrast leakage (see Sec. 2.3).

LOS instability can be traced to both thermal and mechanical effects. LOS drift occurs when the telescope is slewed relative to the Sun. Temperature change causes the telescope structure to expand or contract, resulting in rigid-body motions of the optical components relative to each other. Fortunately, thermal effects are slow and can be corrected. Because HabEx is baselining a laser-truss system to sense and correct slow LOS drift, LOS jitter is the more important performance specification for which to design. Jitter is produced by mechanical disturbance accelerations (from sources such as reaction wheels, cryo-coolers, etc.), exciting modes in the telescope structure and causing rigid body motions (RBM) of the PM, SM, and TM. To correct "slow" jitter (i.e., $<10 \mathrm{~Hz}$ ), HabEx is baselining a low-order Zernike wavefront sensor (ZWFS), similar to the WFIRST low-order wavefront sensor, that can sense and correct LOS jitter on the order of 2.5 mas to $<0.3$ mas per axis. ${ }^{14}$ But, given that the ZWFS cannot correct "fast" jitter at frequencies above $10 \mathrm{~Hz}$, the LOS stability specification has temporal regimes (Table 3). To be conservative, the telescope design is specified to meet the $>10 \mathrm{~Hz}$ requirement.

To design the telescope, it is necessary to convert LOS stability specification into an engineering tolerance (i.e., maximum allowed optical component RBMs). Zemax tolerance analysis of the baseline optical design provides the LOS and WFE sensitivity to RBMs of the PM and SMs relative to the TM for the baseline $F / 2.5$ optical design. ${ }^{15}$ From these sensitivities, an LOS error budget can be allocated for each rigid body degree of freedom (DOF) to keep the on-sky LOS jitter $<0.3$ mas per axis specification. Figure 7 shows one potential error budget allocation.

\subsection{WFE Stability Specification}

WFE stability specifications are driven by the coronagraph. Any temporal or dynamic change in WFE can result in dark hole speckles that produce a false exoplanet measurement or mask a true

Table 3 HabEx LOS specification.

\begin{tabular}{lc}
\hline \hline Temporal frequency & Jitter amplitude per axis \\
\hline$<10 \mathrm{~Hz}$ & $<2$ mas \\
$>10 \mathrm{~Hz}$ & $<0.3$ mas (only required for coronagraphy) \\
\hline
\end{tabular}




\begin{tabular}{|c|c|c|c|c|c|}
\hline \multirow[t]{2}{*}{ Specification } & & & & 0.3 & Mas \\
\hline & & & & \multicolumn{2}{|c|}{ Allocation (one sided PV } \\
\hline Alignment & ZEMAX & Tolerance & Units & RSS & Units \\
\hline PMX-decenter & DX & 2 & Nanometer & 0.043 & Mas \\
\hline PM $Y$-decenter & DY & 2 & Nanometer & 0.042 & Mas \\
\hline PM Z-despace & $\mathrm{DZ}$ & 1 & Nanometer & 0.005 & Mas \\
\hline PM Y-tilt & $\mathrm{TX}$ & 0.5 & Nano-radian & 0.204 & Mas \\
\hline PM $X$-tilt & TY & 0.5 & Nano-radian & 0.200 & Mas \\
\hline PM Z-rotation & $\mathrm{TZ}$ & 0.5 & Nano-radian & 0.027 & Mas \\
\hline SM $X$-decenter & DX & 2 & Nanometer & 0.038 & Mas \\
\hline SM $Y$-decenter & DY & 2 & Nanometer & 0.037 & Mas \\
\hline SM Z-despace & $\mathrm{DZ}$ & 1 & Nanometer & 0.005 & Mas \\
\hline SM $Y$-tilt & TX & 0.5 & Nano-radian & 0.019 & Mas \\
\hline SM $X$-tilt & TY & 0.5 & Nano-radian & 0.019 & Mas \\
\hline SM Z-rotation & $\mathrm{TZ}$ & 0.5 & Nano-radian & 0.002 & Mas \\
\hline RSS LOS error & & & & 0.3 & Mas \\
\hline
\end{tabular}

Fig. 7 RBM tolerance allocation to meet $<0.3$ mas uncorrectable LOS stability specification.

\begin{tabular}{|c|c|c|c|c|c|c|}
\hline \multirow[t]{2}{*}{ Aberration } & \multicolumn{2}{|c|}{ Indices } & \multicolumn{4}{|c|}{ Allowable RMS wavefront error $(\mathrm{nm})$ per mode } \\
\hline & $n$ & $m$ & Charge 4 & Charge 6 & Charge 8 & Charge 10 \\
\hline Tip-tilt & 1 & \pm 1 & 1.1 & 5.9 & 14 & 26 \\
\hline Defocus & 2 & 0 & 0.8 & 4.6 & 12 & 26 \\
\hline Astigmatism & 2 & \pm 2 & 0.0067 & 1.1 & 0.90 & 5 \\
\hline Coma & 3 & \pm 1 & 0.0062 & 0.66 & 0.82 & 5 \\
\hline Spherical & 4 & 0 & 0.0048 & 0.51 & 0.73 & 6 \\
\hline Trefoil & 3 & \pm 3 & 0.0072 & 0.0063 & 0.57 & 0.67 \\
\hline $2^{\text {nd }}$ astig. & 4 & \pm 2 & 0.0080 & 0.0068 & 0.67 & 0.73 \\
\hline $2^{\text {nd }}$ coma & 5 & \pm 1 & 0.0036 & 0.0048 & 0.69 & 0.85 \\
\hline $2^{\text {nd }}$ spher. & 6 & 0 & 0.0025 & 0.0027 & 0.84 & 1 \\
\hline Quadrafoil & 4 & \pm 4 & 0.0078 & 0.0080 & 0.0061 & 0.53 \\
\hline $2^{\text {nd }}$ trefoil & 5 & \pm 3 & 0.0051 & 0.0056 & 0.0043 & 0.72 \\
\hline $3^{\text {rd }}$ astig. & 6 & \pm 2 & 0.0023 & 0.0035 & 0.0034 & 0.81 \\
\hline $3^{\text {rd }}$ coma & 7 & \pm 1 & 0.0018 & 0.0022 & 0.0036 & 1.18 \\
\hline $3^{\text {rd }}$ spher. & 8 & 0 & 0.0018 & 0.0018 & 0.0033 & 1.49 \\
\hline \multicolumn{5}{|c|}{ Garreth Ruane, June 2017} & \multicolumn{2}{|c|}{$\begin{array}{l}\text { Not rejected } \\
\text { First-order rejection } \\
>\text { first-order rejection }\end{array}$} \\
\hline
\end{tabular}

Fig. 8 Wavefront stability required by VVC.

signal. The issue is how large of a WFE a coronagraph can tolerate. A leading candidate is the VVC-N, where $N$ indicates the "charge" or azimuthal shear. Figure 8 summarizes the maximum amount of each Zernike polynomial that can be tolerated by VVC-4 to VVC-10. ${ }^{16}$ The higher the VVC charge, the more low-order error it can reject; however, when the IWA is larger, the throughput is lower. Also, note that Table 8 is reporting the maximum amount for each term if that term was the only error source. In practice, it is necessary to distribute the error between all the terms likely to occur. Currently, HabEx is baselining the VVC-6.

Figure 9 outlines a method for creating a wavefront stability error budget discussed in detail in Refs. 5 and 6. The method derives the error budget to detect an exoplanet based on the total allowable coronagraph contrast leakage to enable a detection at a defined signal-to-noise ratio with specific noise properties. Figure 9 shows the case of an exo-Earth at $10 \mathrm{pc}$. A different design case would yield a different error budget. The exoplanet is detectable as long as WFE instability produces $<40 \mathrm{ppt}$ (parts per trillion) of contrast instability. To be conservative, the HabEx study assumed 30 ppt. The next step is to calculate the sensitivity of the coronagraph's contrast leakage to each Zernike polynomial. Then, using this sensitivity, one must allocate the allowable contrast [on a root sum square (rss) basis] between each Zernike term to produce a wavefront stability error budget.

Figure 10 demonstrates this process for the VVC-6. The first data column shows the VVC6's contrast leakage sensitivity for each Zernike term. As is evident, the VVC-6 is insensitive to 


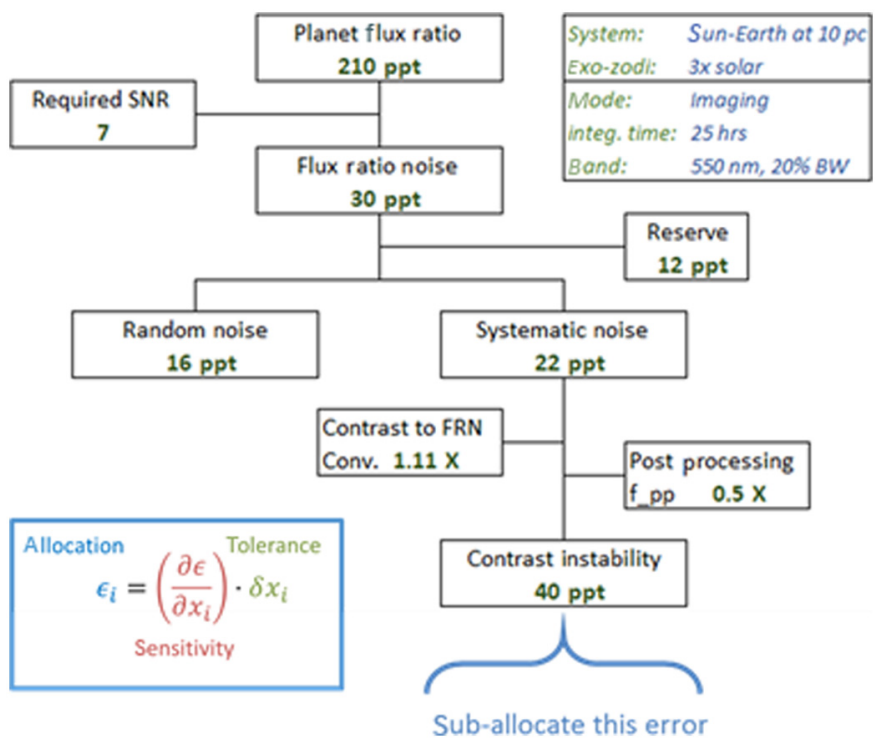

Fig. 9 Wavefront stability error budget development method.

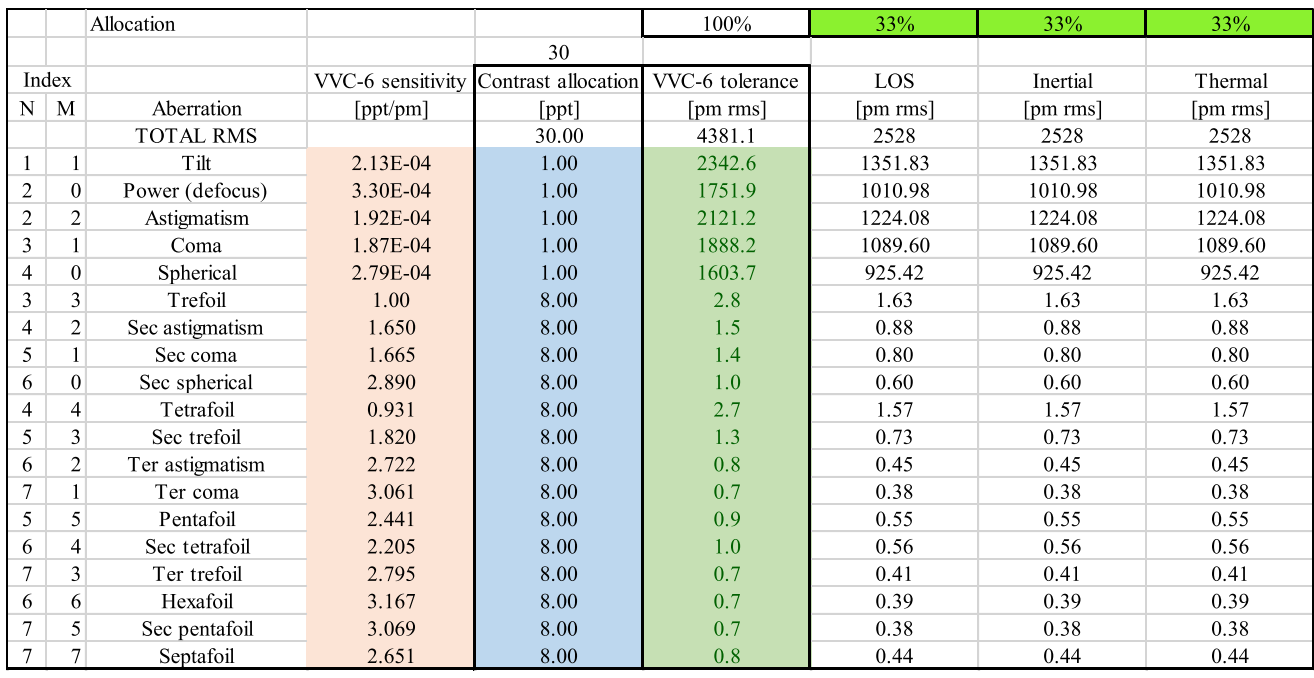

Fig. 10 Allocation of WFE stability between LOS, inertial, and thermal sources.

lower-order error up to spherical and very sensitive to trefoil and higher-order errors. The next column shows a completely arbitrary allocation of contrast: $1 \mathrm{ppt}$ of contrast is allocated to tilt, power, astigmatism, coma, and spherical; and the balance is divided evenly between higher-order Zernike terms. Note that total contrast is an rss of the individual terms. Multiplying these contrast allocations by the coronagraph sensitivity yields an initial total wavefront stability error budget. It is then necessary to suballocate these errors to the actual physical mechanisms by which they arise: LOS jitter, inertial, and thermal deformations. Again, for simplicity, this example assumes a $33 \%$ suballocation to LOS, inertial, and thermal wavefront stability.

WFE instability can arise from both mechanical and thermal sources. Mechanical LOS WFE instability occurs when LOS drift/jitter causes beam-shear on the SM and TM. Because the mirrors are conics, beam shear manifests itself as low-order astigmatism and coma (shear of spherical aberration is coma and subaperture coma appears to be astigmatism). Inertial WFE instability occurs when the PM is accelerated by mechanical disturbances, causing it to react (i.e., bend) against its mounts. Consistent with intuition, the shape of this error is similar to the mirror's static $(X, Y, Z)$ gravity sag (i.e., bending of the mirror when it is exposed to 
a $1 \mathrm{G}$ acceleration in the $X, Y, Z$ directions). Therefore, the shape of the inertial WFE instability can be tailored by adjusting the geometry of the mirror mount design (i.e., 3-versus 6- versus 9-point) mount and location of mounts (i.e., edge versus $70 \%$ radius).

There is one mitigation for LOS and inertial instability. Mechanical motions tend to be periodic, and multiple cycles over an integration period will produce a fixed pattern. If this pattern is $100 \%$ repeatable, it is possible to remove it through "speckle subtraction." Yet, if the motion is not perfectly periodic, there will be a nonrepeatable component that cannot be calibrated and removed. Thus, it is the instability of the mechanical motion (not the motion itself) that is important. Regardless, the best mitigation strategy is to either minimize mechanical disturbances by minimizing mechanical noise, isolate such noise from the telescope via passive isolation, or active sense and control.

Thermal WFE instability occurs when the PM's bulk temperature or temperature gradient changes. If the mirror's CTE is completely homogeneous and constant, then the bulk temperature should only result in a defocus error. However, any inhomogeneity in the mirror's CTE will result in a temperature-dependent WFE. In addition, because CTE is itself temperature dependent, any change in the mirror's thermal gradient will also result in a WFE. Unlike a mechanical WFE, which is mostly low spatial frequency, a thermal WFE can have significant high-spatialfrequency content. Therefore, the best mitigation strategy is to minimize thermal disturbances. This can be done either by passive isolation or active sense and control.

While Fig. 10 demonstrates a simple uniform allocation between Zernike terms and a 33\% allocation among LOS, inertial, and thermal wavefront stabilities, a real error budget for a real telescope/coronagraph architecture must balance the allocation based on the likelihood of each error source occurring. (This process is discussed in Sec. 5 and illustrated for the Baseline HabEx telescope in Fig. 61.)

\section{Telescope Optomechanical Design}

Telescope design is an iterative process. The current design is a result of multiple iterations over a 3 -year period involving numerous trade studies on structure, PM design, PM mounting, where to locate the science instruments, etc. ${ }^{15,17-19}$ These trades were accomplished using STOP analysis to evaluate each iteration's ability to achieve the required performance specifications (LOS and WFE stability). A fundamental rule for the design was that every proposed system, subsystem, or component should be at TRL-6 or higher, except for the primary mirror assembly (PMA) and science instruments. The results are extremely robust. This paper reports performance analysis (LOS, mechanical, and thermal wavefront stability) for the Baseline Rev 3 telescope design.

An ultrastable optomechanical telescope is required to meet the specified WFE and LOS. The baseline telescope architecture achieves this level of performance because of the mass and volume capacities of the planned SLS. SLS mass capacity enables the design of an extremely stiff optomechanical structure that can align the PM, SM, and TM to each other and maintain that alignment. Moreover, SLS volume capacity enables the use of a monolithic-aperture, off-axis PM with no deployments.

\subsection{Baseline HabEx Optical Telescope}

The "baseline" telescope (Fig. 11) consists of the PMA, secondary mirror assembly (SMA), SM tower with integrated science instrument module (ISIM), and stray-light tube with forward scarf. The scarf angle (currently set at $40 \mathrm{deg}$ ) determines the closest angle of observation to the Sun. The tower and baffle tube are the optical bench that maintains alignment between the PMA, SMA, and TMA. The OTA is physically separate from the spacecraft, which includes the solar array sunshield. The size of the solar arrays on the bottom is driven by thermal power requirements during anti-Sun pointing. Instead of reaction wheels, thrusters are used for slewing the observatory, and microthrusters are used for fine pointing control during science observations. The OTA and spacecraft connect only at the interface ring. This ring is also the interface between the payload and the SLS. The forward door is not shown. It is closed for launch to prevent 


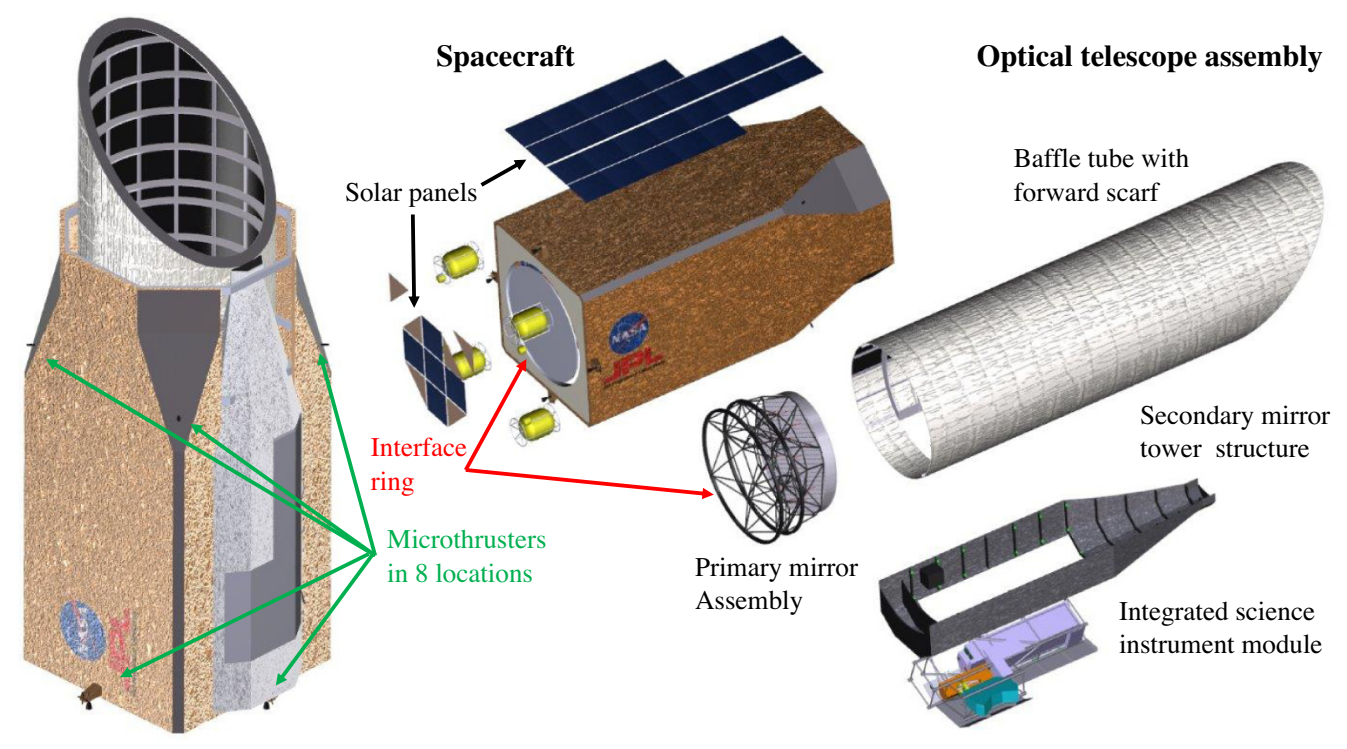

Fig. 11 Baseline HabEx observatory payload.

contamination and provide additional stiffness. In addition, launch locks connect the spacecraft solar panels to the tube for launch.

The baseline observatory is designed for the SLS block $1 \mathrm{~B}$ mass and volume capacities and launch environment. ${ }^{19}$ The payload fits inside the SLS 8.4-m fairing (Fig. 12) with no deployments. The projected total mass (with 30\% reserve) of 14 metric ton (mt) (Table 4) has significant margin against the SLS block-2 capacity of $44 \mathrm{mt}$ to the Sun-Earth Second Lagrange orbit (SE-L2). SE-L2 is the preferred orbit for HabEx because of its thermal and mechanical stability. The telescope and spacecraft structures are designed to have a first mode higher than $10 \mathrm{~Hz}$ and to survive a $3.5 \mathrm{-g}$ axial and 1.5-g lateral launch load. Table 5 lists the current best mass estimate for each of the telescope's major elements.

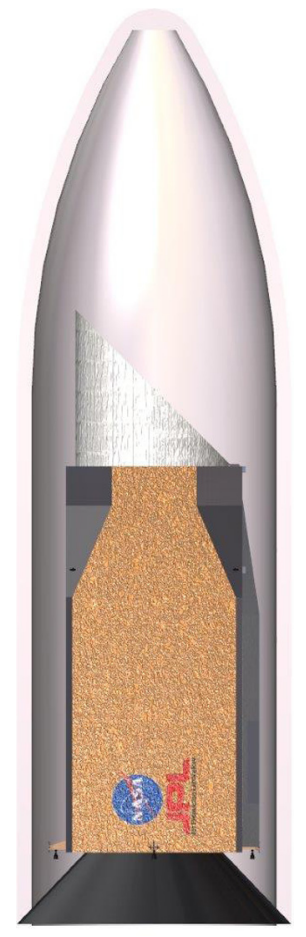

Fig. 12 Payload fits in SLS $8.4 \mathrm{~m}$ fairing. 
Stahl et al.: Habitable-Zone Exoplanet Observatory baseline 4-m telescope...

Table 4 HabEx observatory payload mass estimate.

\begin{tabular}{lccc}
\hline \hline Element & Current best estimate $(\mathrm{kg})$ & Reserve $(\mathrm{kg})$ & Total $(\mathrm{kg})$ \\
\hline Telescope & 3431 & 1029 & 4460 \\
Science instruments & 1164 & 499 & 1663 \\
Spacecraft & 4500 & 1350 & 5850 \\
Interface ring & 210 & 63 & 273 \\
Payload adapter feature & TBD & - & - \\
Payload dry mass & 9305 & 2941 & 12,246 \\
Propellant & 1700 & - & 1700 \\
Payload wet mass & 11,005 & - & 13,946 \\
\hline \hline
\end{tabular}

Table 5 HabEx optical telescope mass estimate.

\begin{tabular}{lc}
\hline \hline Component & Current Best Estimate $(\mathrm{kg})$ \\
\hline PM (with launch locks) & 1453 \\
PM support & 865 \\
SMA & 11 \\
TM assembly & 65 \\
Tower and baffle tube & 982 \\
Forward door & 55 \\
Total OTA mass & 3431 \\
\hline \hline
\end{tabular}

\subsection{Optical Telescope Structure}

The structure is the foundation for the entire telescope. It is the optical bench to which the optical components and science instruments are attached. To meet the required performance specifications, the optical telescope structure has the critical function of placing the PM, SM, and TM at the locations specified by the optical design and keeping them at those locations with sufficient stability (see Secs. 2.2 and 2.3). This is accomplished by making the structure as stiff as possible and by minimizing the disturbances to which the structure is exposed, given that the optical design is a TMA and three of the four science instruments share a common TM. The TM location is fixed, and the PM and SM alignments are adjusted relative to the TM. As presented in Sec. 4, STOP modeling predicts that the baseline structure meets the LOS jitter and WFE stability specifications for a specified microthruster disturbance profile.

Per Lake and Levine ${ }^{20}$ WFE is proportional to rms magnitude of the applied inertial acceleration divided by square of the structure's first mode frequency. Thus, for a given stiffness structure, a $10 \times$ reduction in acceleration results in a $10 \times$ WFE reduction. However, for a given acceleration level, a 10× increase in first mode frequency results in a 100 $\times$ WFE reduction. Thus, one should design the structure to be as stiff as possible. As shown in Fig. 13, for a 100-Hz structure, picometer displacement is achieved by limiting noise acceleration to micro-g levels.

While not designed to meet the requirements of a UV/Optical/Near-IR (UVOIR) exoplanet science mission, JWST is an example of what is possible. The JWST SM support structure has a first mode of $\sim 7.5 \mathrm{~Hz}$ at its operating temperature of $50 \mathrm{~K}$. When excited by the reaction wheels, SM motion can impact JWST's LOS and WFE specifications (Fig. 14). ${ }^{21}$ However, because of

J. Astron. Telesc. Instrum. Syst. $\quad$ 034004-12 Jul-Sep 2020 • Vol. 6(3) 


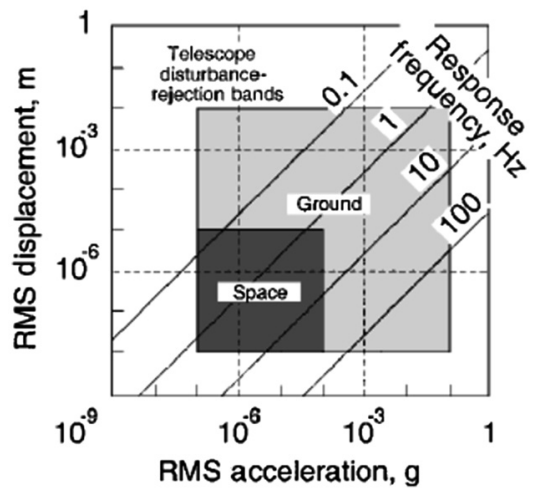

Fig. 13 Error versus load.
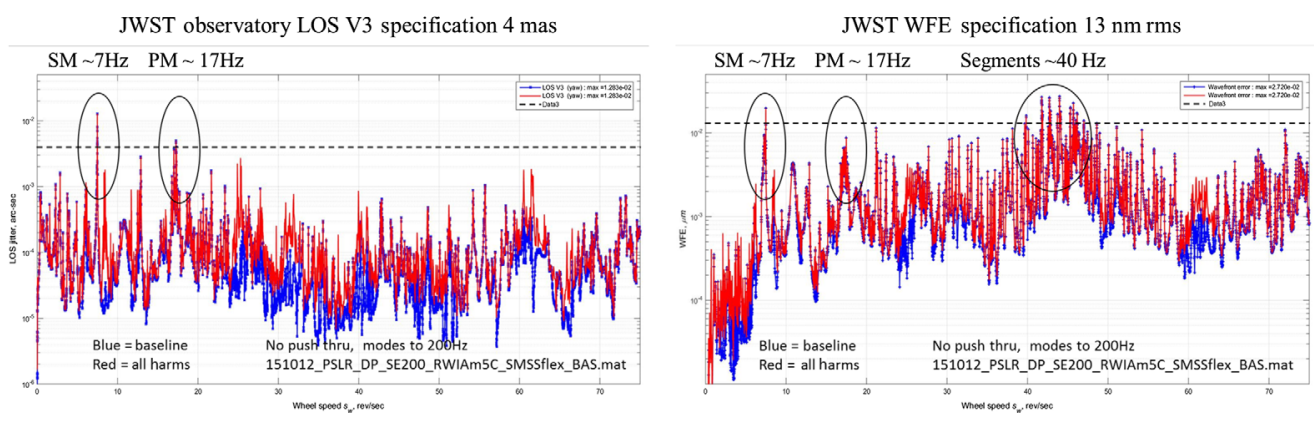

Fig. 14 JWST cryogenic LOS and WFE performance predictions.

dampening, a warm JWST may have LOS stability consistent with the HabEx specification of $<0.5$ mas per axis. ${ }^{22}$ To provide design margin, the HabEx goal was to design a secondary support structure with a first mode frequency of $\sim 28 \mathrm{~Hz}$ (i.e., 4x stiffer than JWST). But the PM's $2.5 \mathrm{~F}$ /\# makes this challenging. HabEx's PM-to-SM separation is $\sim 9 \mathrm{~m}$ (compared to $\sim 7 \mathrm{~m}$ for JWST).

The baseline structure is the result of multiple design iterations. ${ }^{23}$ An initial standalone truss tower concept had an unacceptably low first-mode frequency of $\sim 2 \mathrm{~Hz}$. Connecting it to the stray-light baffle tube yielded modes of 11 to $30 \mathrm{~Hz}$. In addition to straylight suppression, the internal baffles provide stiffness. However, because the optical design is off-axis, the baffle rings are not continuous, thus gussets were added to the tower structure, which span the baffle gaps. These gussets eliminated the need for a truss structure, reducing mass and opening the space for instruments (Fig. 15). The SMA attaches to the top of the tower and the bottom contains the ISIM. The tower and tube use TRL-9 composite material M46J with quasi-isotopic laminate properties of $25 \% 0 \mathrm{deg}, 50 \% 45 \mathrm{deg}$, and $25 \% 90 \mathrm{deg}$, as well as a density of $1.58 \mathrm{~g} / \mathrm{cm}^{3}$.

As shown in Fig. 11, the telescope is a standalone structure separate from the spacecraft. The spacecraft surrounds the telescope without physically touching it, except at the interface ring (which also connects to the launch vehicle payload adapter fixture). This configuration minimizes the propagation of mechanical disturbances from the spacecraft into the telescope and provides thermal shielding of the telescope while minimizing heat leaks. In fact, STOP modeling predicts that thermal isolation is too good. To increase the PM cold bias, it was necessary to remove portions of the spacecraft anti-Sun structure. While the PM and SM have active thermal control, the structure does not (although it could be added). The structure is not actively heated because the telescope includes a laser truss system that maintains alignment between the PM, SM, and TM. The laser truss bandwidth is sufficient to sense and correct slow thermal drifts, and its noise uncertainty is sufficient to meet the LOS Jitter and LOS WFE stability specifications. Finally, while STOP modeling predicts that the integrated telescope/spacecraft payload's mechanical performance meets the LOS Jitter and LOS WFE stability specifications (Sec. 4), 

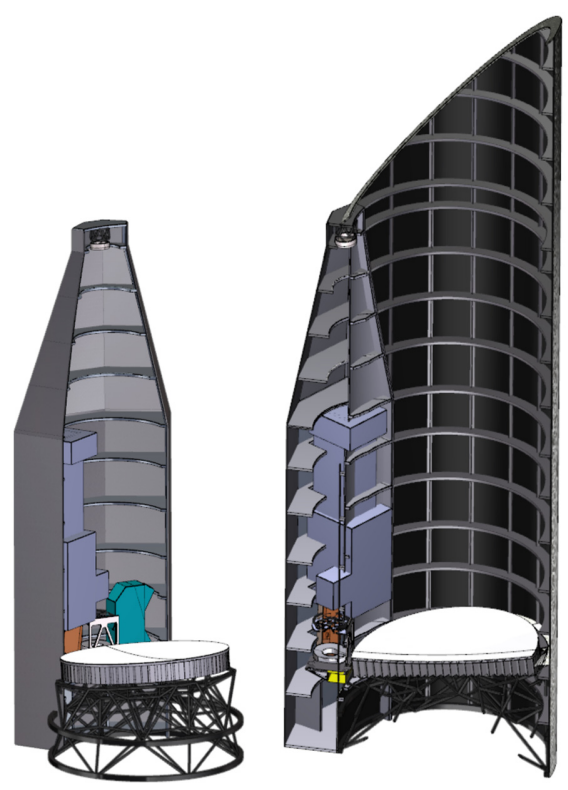

Fig. 15 Gussets stiffen tower/tube structure.

margin can be obtained by adding passive damping (such as Northrop's JWST passive struts) or active vibration isolation (such as Lockheed's disturbance free payload technology) to the interface ring.

\subsection{PMA}

A PMA is an integrated optomechanical system consisting of a mirror substrate (its mount, support structure, and launch lock system) and an active thermal control system. A PMA must be designed as a whole element because interactions among PMA elements determine the telescope's ability to meet its performance specifications (Table 1). In designing the PM, the HabEx design team followed proven design practices and assumed it would be fabricated using processes proven on previous 1- to 2.4-m TRL-9 mirrors (e.g., Kepler, WFIRST, and Hubble). Furthermore, the design team improved the Astrophysics Division's funded technology development to design and fabricate a 4-m-class ultrastable UVOIR PM (e.g., Advanced Mirror Technology Development, AMTD, project and Predictive Thermal Control Study, PTCS). ${ }^{24-28}$ The baseline HabEx design is a monolithic 4-m ZERODUR ${ }^{\circledR}$ open-back iso-grid mirror. An alternative design is a ULE ${ }^{\circledR}$ closed-back hex-core mirror. ZERODUR ${ }^{\circledR}$ was selected for the baseline design because at $4 \mathrm{~m}$ it has a higher TRL than $\mathrm{ULE}^{\odot}$.

\subsubsection{Monolithic versus segmented}

The single most important design decision is whether to make the PM monolithic or segmented. The current largest space mirror is the 2.4-m monolith on Hubble; however, once Webb flies, it will carry a $6.5-\mathrm{m}$ segmented mirror. It is much easier to achieve the ultrastable wavefront required for coronagraphy with a monolithic mirror than with a segmented mirror. As discussed in Sec. 2.3 and shown in Fig. 10, habitable-zone coronagraphy requires a wavefront with picometer-level stability in the mid-spatial frequency regime (i.e., 2 to 10 cycles per aperture). To meet this specification, a segmented aperture mirror must align and phase its segments to each other and maintain that phasing with a stability of a few pm. ${ }^{5,25,29-32}$ A monolith has no such requirement.

With its $2.0-\mu \mathrm{m}$ diffraction limit, JWST was obviously not designed to meet the requirements of a UVOIR exoplanet science mission. But JWST defines the current state of the art. As shown in Fig. 14, JWST's primary mirror segment assemblies (PMSA) are predicted to have 20-nmrms rocking modes in the 40- to 50-Hz range, which impact WFE. In addition, the JWST PMA is predicted to have an $\sim 40-\mathrm{nm}-\mathrm{rms}$ "wing flap" mode at $\sim 17 \mathrm{~Hz}$ (Fig. 14), driven entirely by 
back-plane stiffness. ${ }^{21}$ While JWST was not designed for coronagraphy, its design can be improved. Increasing the backplane stiffness from 17 to $170 \mathrm{~Hz}$ could potentially reduce the wing flap amplitude to $\sim 400 \mathrm{pm} \mathrm{rms}$. And an $\sim 10 \times$ improvement in WFE might be achieved by operating JWST while warm (because of dampening). But to get into the 1 to $2 \mathrm{pm}$ stability requires increasing its vibration isolation from 70 to $140 \mathrm{~dB} .^{22}$ All of this complexity and engineering difficulty is avoided with a monolithic mirror. Two other examples of segmented aperture telescopes are the Large Aperture Mirror Program (LAMP) and Segmented Mirror Telescope (SMT). LAMP (Fig. 16) was a ground demonstration of a 4-m, actively controlled, segmented mirror. Each of its seven 2-m segments were $~ 17-\mathrm{mm}$ thick and attached to surface control actuators mounted in a stiff, lightweight, graphite-epoxy, and back-up structure. A total of 312 surface control and 42 segment position actuators enabled the LAMP mirror to be controlled to a wavefront accuracy of $<100 \mathrm{~nm}$ rms (i.e., diffraction limit of $1.3 \mu \mathrm{m}$ ). ${ }^{33}$ SMT (Fig. 17) is a 3-m telescope with six hexagonal petals. Each segment has more than 100 actuators for surface control, as well as three fine and six coarse actuators to bring the segments into alignment after deployment. ${ }^{34}$ SMT was designed to be diffraction limited at $0.5 \mu \mathrm{m}$ but only achieved $5 \mu \mathrm{m}$ because of structural and thermal instability.

Another important design trade is aperture diameter, because size drives science yield. While the SLS 8.4-m fairing can accommodate an aperture larger than $6 \mathrm{~m}, 4 \mathrm{~m}$ was selected for programmatic reasons. A 4-m PM can be affordably manufactured using existing infrastructure available at multiple potential suppliers. Schott has existing infrastructure to melt, cast, and lightweight-machine 4.2-m ZERODUR ${ }^{\circledR}$ mirror substrates (Fig. 18). ${ }^{35}$ Similarly, Corning has

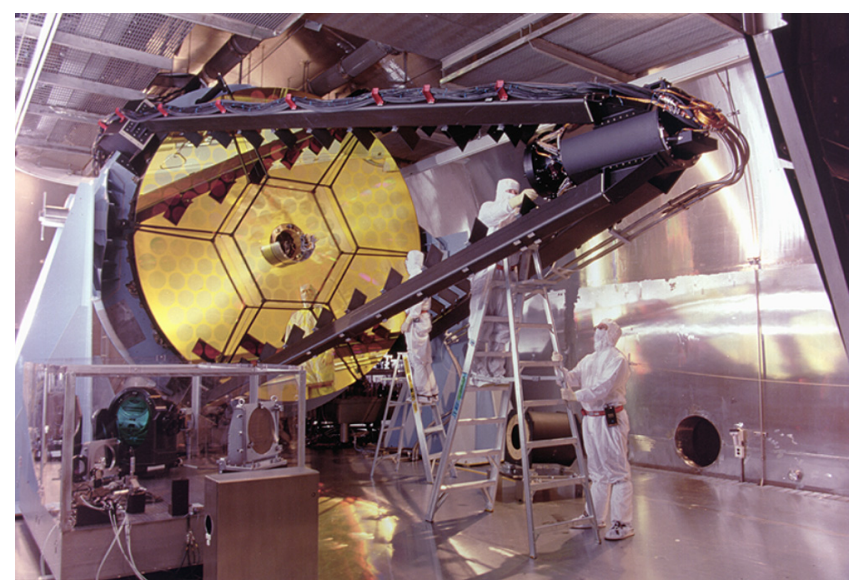

Fig. 16 4-m LAMP telescope.

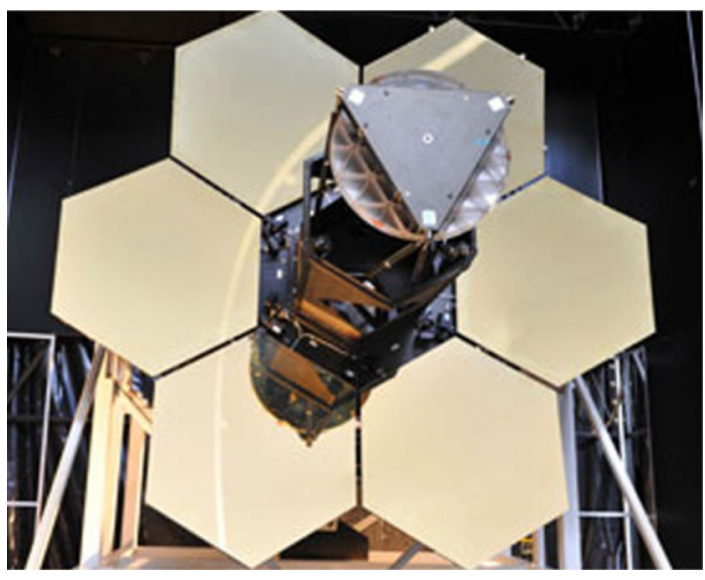

Fig. 17 3-m SMT telescope. 


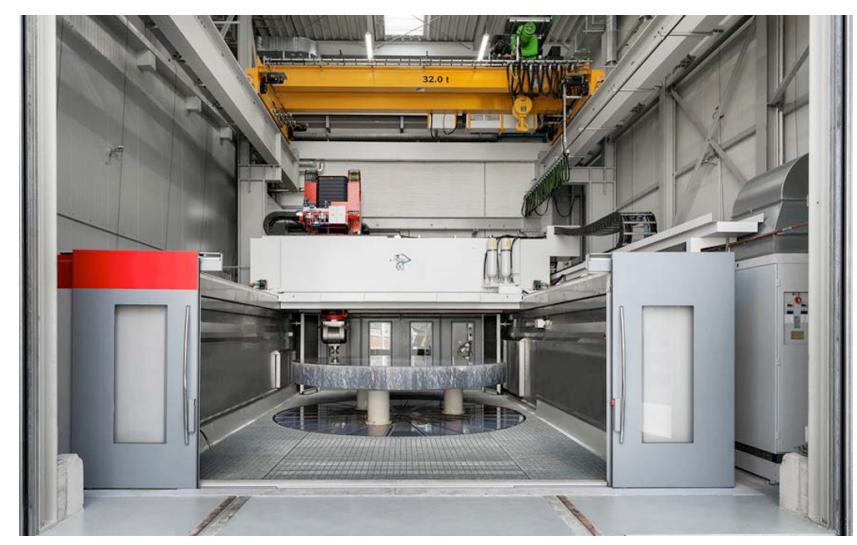

Fig. 18 Schott Glass 5-m, 5-axis CNC machine center-loaded with a 4.5-m glassy ZERODUR ${ }^{\circledR}$ blank.

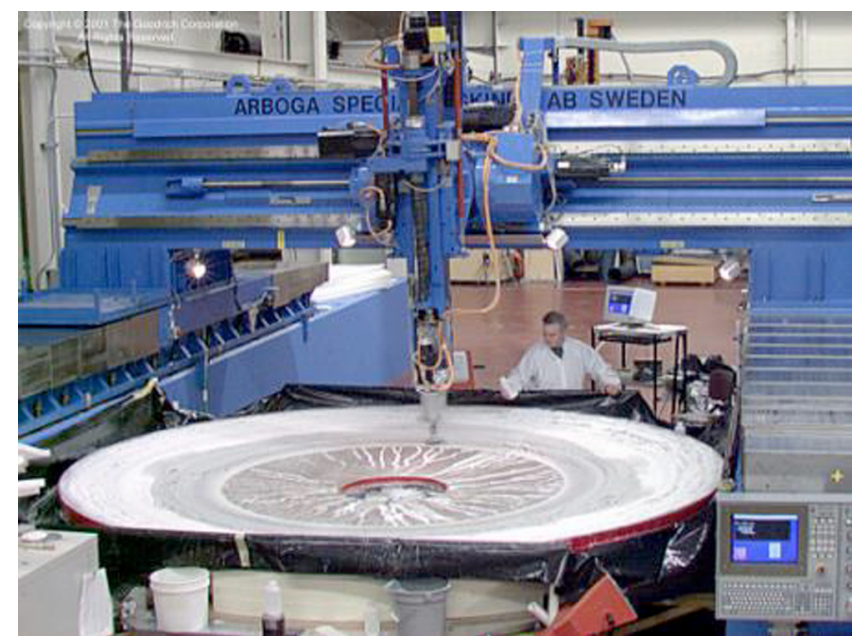

Fig. 19 Collins Aerospace computer-controlled manufacture of 4.2-m SOAR PM.

infrastructure to assemble 4-m "class" ULE ${ }^{\circledR}$ mirror substrates via either frit bond or low temperature-fusion (LTF). And multiple organizations have existing infrastructure to grind and polish 4-m class substrates into space mirrors, including: Collins Aerospace in Danbury, Connecticut; L3/Brashears in Pittsburgh, Pennsylvania; Harris Corporation in Rochester, New York; Arizona Optical Systems and the University of Arizona in Tucson, Arizona; and RESOC outside of Paris, France. Figure 19 shows the 4.2-m Southern Astrophysical Research Telescope (SOAR) PM being polished via computer control at Collins Aerospace. ${ }^{36}$ In addition, several organizations are considering, planning, or implementing the ability to coat 4- to 6-m class mirrors by scaling up the proven process demonstrated on $2.5-\mathrm{m}$ class mirrors, including: Collins Aerospace, ${ }^{36}$ ZeCoat Corporation, ${ }^{37}$ and Harris Corporation. Because 4-m mirrors can be manufactured by multiple organizations using existing infrastructure and processes, they are relatively affordable and low risk.

\subsubsection{PM material}

Selecting the mirror material involves thermal, mechanical, and programmatic considerations. For HabEx, a key metric is CTE. CTE and CTE homogeneity are important because they determine how the mirror's shape deforms as a function of bulk temperature or thermal gradient changes. Such deformations impact the telescope's ability to meet its diffraction-limited performance and wavefront-stability specifications. The typical approach for mitigating this risk is to 
dL/L customized ZERODUR cases

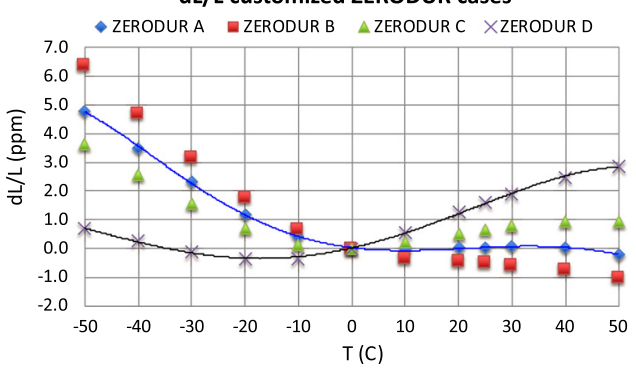

(a)

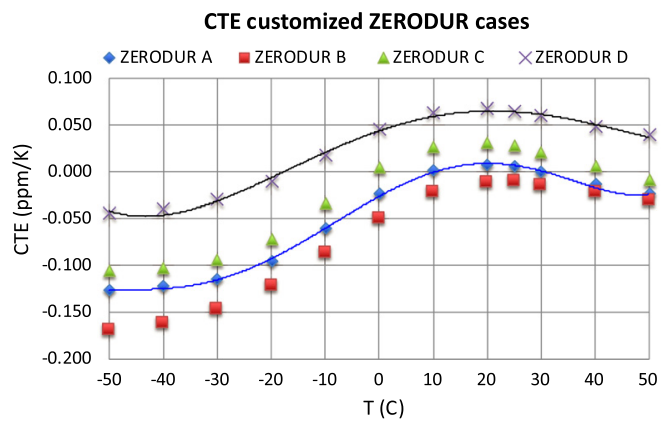

(b)

Fig. 20 Schott can tailor its Zerodur ${ }^{\circledR}$ ceramic to have (a) a zero slope $d L / L$ or (b) zero CTE at temperatures ranging from $\sim-20^{\circ} \mathrm{C}$ to $+40^{\circ} \mathrm{C}$ with an uncertainty of $\pm 4^{\circ} \mathrm{C}$. ${ }^{38}$
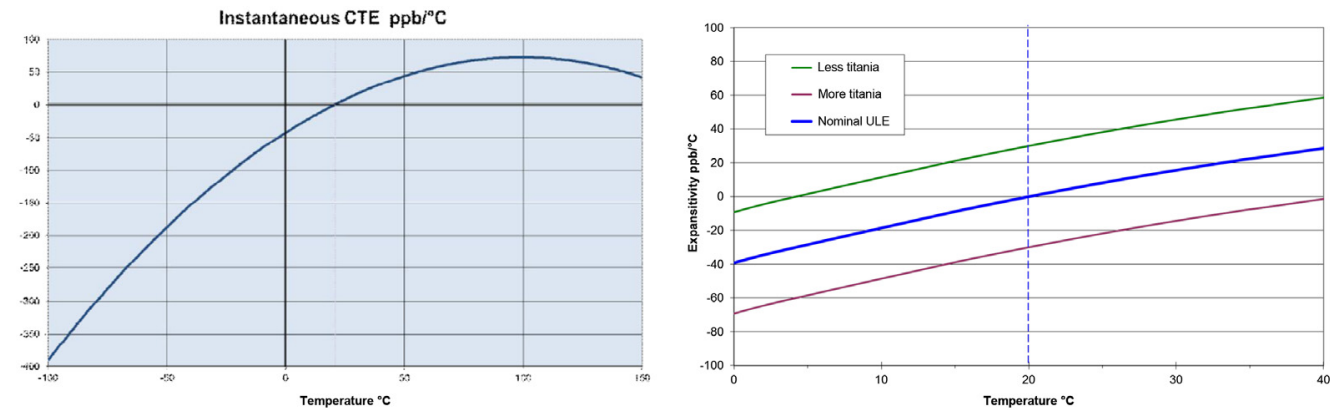

Fig. 21 Corning can tailor its $\mathrm{ULE}^{\circledR}$ glass to have a zero CTE at temperatures ranging from $\sim 5^{\circ} \mathrm{C}$ to $40^{\circ} \mathrm{C}$ based on the amount of titanium dopant with an uncertainty of $\pm 4^{\circ} \mathrm{C}$. Less titanium shifts the zero-CTE to lower temperatures. More titanium shifts zero-CTE to higher temperatures. ${ }^{39}$

select a material with zero CTE and extreme homogeneity. A second approach is to measure the mirror's shape change between its manufacture and operational temperatures and then "cryo-null figure" the mirror to have the desired surface shape at the operational temperature. This is the approach taken for the JWST beryllium mirrors. A final approach is to actively control the mirror's on-orbit shape.

The HabEx telescope design team evaluated ZERODUR ${ }^{\circledR}$ and ULE $^{\circledR}$. Both materials are TRL-9 with multiple mirrors currently flying in space. Both Schott and Corning can tailor their respective material's zero CTE temperature (Figs. 20 and 21) and both claim similar CTE homogeneity (i.e., $\pm 5 \mathrm{ppb}$ ). Therefore, a mirror manufactured from either material should have similar thermal performance. But the real impact of this design decision is architectural-whether the mirror is open-backed or closed-back. Because ZERODUR ${ }^{\circledR}$ is a ceramic, it must be machined from a single boule, resulting in an open-back architecture. By comparison, $\mathrm{ULE}^{\circledR}$ is a glass and can be assembled via frit bonding or LTF processes into a closed-back architecture. The advantage is that closed-back mirrors have significantly higher stiffness. Yet, at the same time, because a ZERODUR ${ }^{\circledR}$ mirror is machined from a single boule, their CTE distribution can be smoother and more homogeneous.

\subsubsection{Primary mirror substrate design}

The most important design criterion is mirror stiffness. Stiffness enables performance and simplifies manufacture. When the mirror's stiffness is higher, it is easier to produce the smooth surface needed to achieve 400-nm diffraction limited performance. The higher the mirror's stiffness, the smaller its gravity sag or self-weight deflection, which results in a smaller inertial error and lowers the potential of a significant G-release error. Also, a stiffer mirror is easier to handle (i.e., mounting to machinery or simply turning over), which reduces fabrication risk. 
Inertial WFE is the deformation that a mirror experiences when it is accelerated against its mount. To first order, it is a scaled vector-superposition of the mirror's $(X, Y, Z) 1 \mathrm{G}$ gravity sag. For example, if a mirror has $25 \mu \mathrm{m}$ of $1 \mathrm{G}$ gravity sag, it will have $25 \mathrm{pm}$ of deformation when accelerated by 1 micro-G. Note that inertial WFE is not the same as resonant WFE. Resonant WFE typically has a large amplitude and occurs only at specific temporal frequencies. Inertial WFE, on the other hand, occurs at all nonresonant temporal frequencies. Again, JWST provides a definitive example of inertial error. JWST's $220-\mathrm{Hz}$ open-back beryllium PM segments on a three-point mount have a horizontal gravity sag of $\sim 200 \mathrm{~nm}$. When driven (in a horizontal configuration) at $87.3 \mathrm{~Hz}$, they have an inertial astigmatic WFE of $\sim 220 \mathrm{~nm}$ per G of driving force. ${ }^{40}$ There are two approaches for minimizing inertial WFE: make the mirror as stiff as possible to minimize its gravity sag and/or minimize the mirror's exposure to acceleration by minimizing thruster noise or isolating the mirror from such noise.

G-release error, as discussed in Sec. 2.1.3, is the error between a mirror's 1-G shape and its on-orbit $0-G$ shape. The risk that a mirror will have significant G-release error is correlated with the mirror's stiffness. The lower the mirror's stiffness, the greater will be its 1-G gravity sagand the greater will be the uncertainty in the fabrication process's ability to characterize and compensate for or back-out that gravity sag. Thus, it is best to make the mirror as stiff as possible.

For a 400-nm diffraction-limited telescope, G-release error should be $<4 \mathrm{~nm}$ rms. While TRL-9 techniques exist to characterize self-weight deflection to $<10 \mathrm{~nm} \mathrm{rms}$, it is also possible to mitigate the risk of G-release error via an active mirror (e.g., PM, SM, or deformable flat mirrors). An active PM can easily correct 50 to $200 \mathrm{~nm}$ rms of error using TRL-9 technology. While they were never used, the Hubble PM had 24 actuators to mitigate the risk of astigmatic G-release error. ${ }^{41}$ The Webb PM has 18 actuators to mitigate the risk of segment-radius matching error and segment-level astigmatism.

Two TRL-9 methods for characterizing and removing self-weight deflection to $<10 \mathrm{~nm} \mathrm{rms}$ are a metrology mount that compensates for or off-loads the effect of gravity during testing and testing the mirror under multiorientation to mathematically solve for gravity deformation separate from surface shape. Regarding gravity off-loading, two implementations are multipoint mount and air bag. For mid-quality mirrors, air bags are adequate. For high-precision mirrors, multipoint mounts are preferred. Multipoint metrology mount technology was developed with NASA funding in the 1970s for the Large Space Telescope Program (Hubble). ${ }^{41-43}$ The Hubble PM's 7.6- $\mu \mathrm{m}$ self-weight deflection was characterized to an accuracy of $1.4 \mathrm{~nm}$ rms using a 135point metrology mount. ${ }^{41,44,45}$ Regarding gravity orientation testing, two methods are the vertical face-up/face-down test ${ }^{46}$ and the horizontal rotation testing. ${ }^{36,47,48}$ Typically, a multipoint mount or air bag is used for in-process metrology, and a multiorientation test is used for final metrology. The Kepler PM was tested using an air bag, a 108-point metrology mount, and a face-up/facedown gravity orientation test. ${ }^{46}$ The air bag was estimated to off-load gravity sag with an uncertainty of $5.6 \mathrm{~nm}$ rms. The difference between the air bag test and multipoint mount test was $16.4 \mathrm{~nm} \mathrm{rms}$. The difference between the air bag and face-up/face-down test was $18.4 \mathrm{~nm}$ rms. In both cases, the largest component of this difference was spherical aberration. By inference, the difference between the multipoint mount and the up/down test was $8.3 \mathrm{~nm} \mathrm{rms}$.

Whether the PM is active or passive, it is important to make it as stiff as possible to minimize its gravity sag (because when the amplitude is smaller, it is easier to characterize and remove the amplitude from the $1 \mathrm{G}$ metrology data). For any given mirror material, mirror gravity sag is proportional to geometry, mass density, and mount interface:

$$
1 \text { G Gravity Sag } \sim C_{\mathrm{SP}}\left(\frac{D^{4}}{t^{3}}\right) \rho_{\mathrm{AD}} \sim 1 /(2 \pi f)^{2},
$$

where $C_{\mathrm{SP}}$ is the mount support configuration constant, $D$ is the mirror substrate diameter, $t$ is the mirror substrate thickness, $\rho_{\mathrm{AD}}$ is the mirror substrate mass areal density, and $f$ is the mirror substrate first mode frequency.

To minimize gravity sag for a 4-m mirror, one must make the mirror as thick as possible and with the lowest areal density possible. For $\mathrm{ULE}^{\circledR}$, the state-of-art thickness is $\sim 30 \mathrm{~cm}$. Specialized abrasive waterjet machines can cut core elements as thick as $28 \mathrm{~cm} .{ }^{49}$ These elements can be frit bonded or low-temperature fused to form a mirror substrate. To make thicker mirrors, 
the AMTD project successfully demonstrated a stack and seal process by manufacturing a 40-cm-thick test mirror. ${ }^{27,28,49}$ For ZERODUR ${ }^{\circledR}$, Schott has demonstrated 42-cm-thick substrates and they are working to produce 45 -cm-thick mirrors. ${ }^{35}$ Other design elements that impact stiffness include: face-sheet thickness; whether not the back of the mirror is open, closed, or partially closed and thickness of the back-sheet; and geometry of the core structure (i.e., iso-grid, rectilinear-grid, or hex-grid), pocket size, core wall thickness, etc. For example, hex-grid is more mass efficient but less stiff than iso-grid. Thus, hex-grid is typically used for closed-back mirrors (because the back sheet adds stiffness), and iso-grid is typically used for open-back mirrors. Also, the mirror mount geometry is important. A complete discussion of mirror design methodology can be found in Yoder and Vukobratovich. ${ }^{41}$

The HabEx design team conducted multiple trade studies with literally hundreds of variations to optimize the stiffness, mass, and gravity sag of candidate open-back ZERODUR ${ }^{\circledR}$ and closed-back ULE ${ }^{\circledR}$ PMs. Design parameters traded included facesheet thickness, mirror thickness, core wall thickness, core pocket size, core geometry, and mount geometry (i.e., edge mount $R=100 \%$ versus $R=80 \%$ or $67 \%) .{ }^{50-54}$ Early in the design process, the goal was to achieve the highest first-mode frequency possible. The reason was to minimize inertial WFE stability caused by reaction wheels. However, once microthrusters with their significantly lower noise properties were baselined, the emphasis shifted to optimizing thermal WFE stability, inertial WFE stability, and demonstrated manufacturability. ZERODUR ${ }^{\circledR}$ was selected as the baseline material because Schott had demonstrated a routine ability to fabricate $4.2-\mathrm{m}$ diameter ZERODUR ${ }^{\circledR}$ substrates and turn them into lightweight structures via their ELZM machining process. Furthermore, a 1.2-m ELZM owned by Schott and tested at NASA Marshall Space Flight Center (MSFC) showed better thermal stability than a $1.5-\mathrm{m} \mathrm{ULE}^{\circledR}$ mirror manufactured as part of the AMTD project. ${ }^{55}$ Part of ZERODUR ${ }^{\circledR}$ CTE advantage over ULE is that the mirror is fabricated from a single boule of material. Thus, a ZERODUR ${ }^{\circledR}$ mirror has a more homogeneous CTE distribution than a mirror assembled from multiple $\mathrm{ULE}^{\circledR}$ components.

The baseline ZERODUR ${ }^{\circledR}$ mirror assembly provides an excellent balance between mass and stiffness. The substrate has a flat-back geometry with a 42-cm edge thickness and mass of $\sim 1400 \mathrm{~kg}$ (Fig. 22). The mirror's free first mode frequency is $88 \mathrm{~Hz}$. Its mounted first-mode frequency is $70 \mathrm{~Hz}$. Mass is important because it provides thermal capacity for a thermally stable mirror. In addition, mass allows for local stiffening of the substrate to minimize gravity sag. ${ }^{50}$ The mirror substrate geometry and hexapod mount designs were optimized to produce the most uniform $X, Y, Z$ gravity sag deformation possible. The mirror is attached at three edge locations to a hexapod-mount system. This geometry was selected to allow defocus and minimize spherical gravity sag based on VVC aberration sensitivity (Fig. 8). Figure 23 shows the baseline mirror's predicted $1-G$ surface gravity sag in global telescope $X, Y, Z$ coordinate system.

As a matter of completeness, other substrate designs considered include an MSFC 45-cmthick closed-back ULE ${ }^{\circledR}$ mirror with total mass of $1388 \mathrm{~kg}$ and first mode frequency of $180 \mathrm{~Hz},{ }^{52}$ a Harris Corporation 40-cm-thick closed-back ULE ${ }^{\circledR}$ mirror with total mass of $440 \mathrm{~kg}$ and first mode frequency of $137 \mathrm{~Hz}$, developed under the AMTD project, ${ }^{56}$ a Schott AG ELZM on-axis 34-cm-thick design with $718 \mathrm{~kg}$ and $\sim 80 \mathrm{~Hz}$ first mode, ${ }^{57}$ and a Collins Corp ZERODUR ${ }^{\circledR}$ shaped-back mirror with mass of $1200 \mathrm{~kg}$ and first mode frequency of $120 \mathrm{~Hz}{ }^{36}$

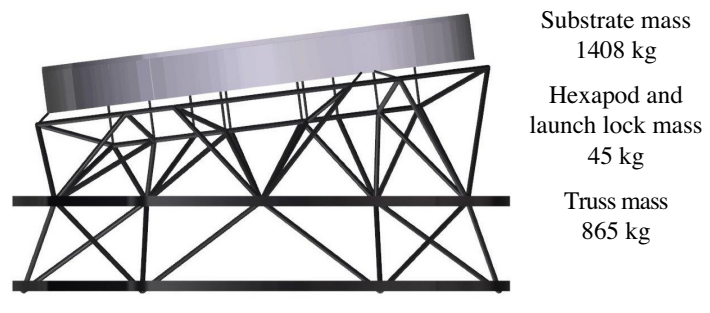

Fig. 22 PMA. 

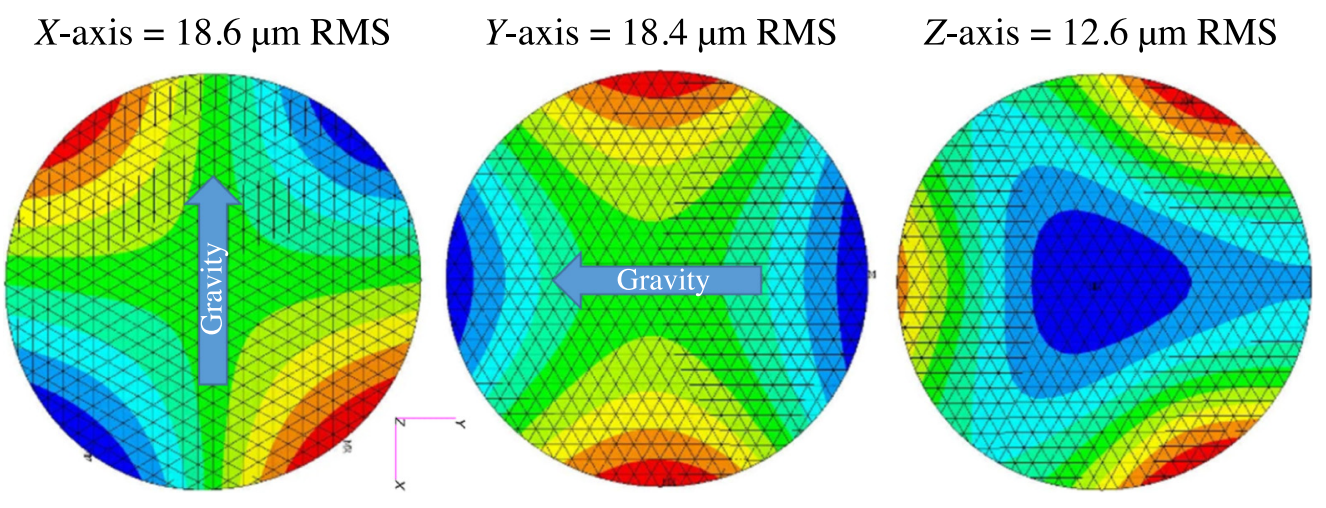

Fig. 23 PM gravity sag (surface).

\subsubsection{PM support structure}

The PM support structure is a simple truss. It is specified to be manufactured from TRL-9 M46J with quasi-isotopic laminate properties of 25\% $0 \mathrm{deg}, 50 \% 45 \mathrm{deg}$, and $25 \% 90 \mathrm{deg}$, as well as a density of $1.58 \mathrm{~g} / \mathrm{cm}^{3}$ with a total mass of $\sim 865 \mathrm{~kg}$ (Fig. 22). As discussed earlier in this section, JWST's PMA is predicted to have a flapping mode at $17 \mathrm{~Hz}$, which, as discussed in Sec. 2.2, is above the anticipated ZWFS $10-\mathrm{Hz}$ control frequency. Therefore, to minimize WFE stability, the HabEx PM hexapod supports and truss structure are designed for its rigid body and bending modes to be above $40 \mathrm{~Hz}$. For example, Figs. 24 and 25 show a $43.5-\mathrm{Hz}$ rocking mode and a $50-\mathrm{Hz}$ bouncing mode.

Finally, the PM truss structure is designed to accommodate a launch constraint system consisting of 18 axial and 12 radial launch locks (Fig. 26). While ZERODUR ${ }^{\circledR}$ is suitable for applications with mechanical loads from 4.3 to $14.5 \mathrm{psi}$ and when manufactured using Schott's standard surface etch protocol can withstand up to $17.4 \mathrm{~K}$ psi for short durations, ${ }^{58}$ standard engineering practice is to limit the maximum launch load to 600 psi. The HabEx design team modeled stress experienced by the baseline PM when exposed to different launch acceleration loads with and without a launch lock system (Table 6). Without a constraint system, launch stress

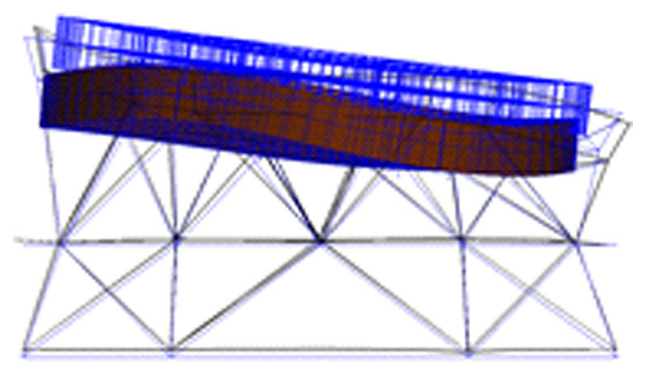

Fig. 24 43.5-Hz rocking mode.

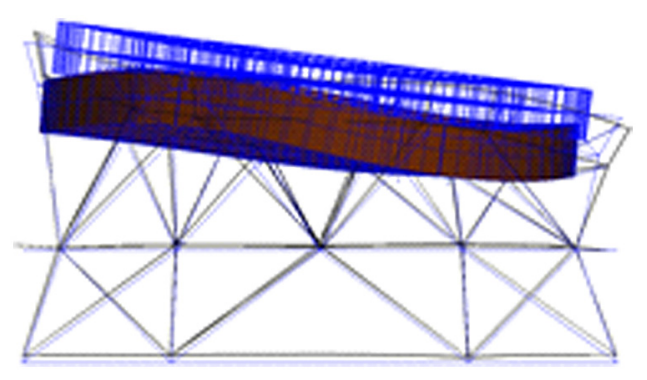

Fig. 25 50- $\mathrm{Hz}$ bouncing mode. 


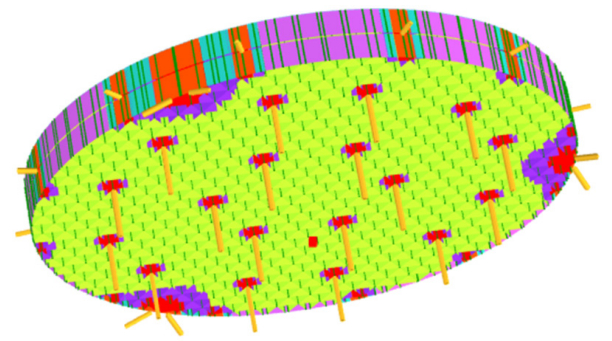

Fig. 26 PM launch constraint system has 18 axial and 12 radial launch locks.

Table 6 Baseline HabEx 4-m PM launch stress.

\begin{tabular}{ccccc}
\hline \hline \multicolumn{2}{l}{ Acceleration loads $(\mathrm{G})$} & & \\
\cline { 1 - 2 }$X$ & $Y$ & $Z$ & No-lock stress (psi) & Locked stress (psi) \\
\hline 0.5 & 0.0 & 6.0 & 995 & 197 \\
0.0 & 0.5 & 6.0 & 959 & 160 \\
2.0 & 0.0 & 3.5 & 702 & 297 \\
0.0 & 2.0 & 3.5 & 657 & 233 \\
\hline \hline
\end{tabular}

of as much as 1000 psi is concentrated at the three hexapod attachment locations (Fig. 27). And, while according to Schott a Zerodur ${ }^{\circledR}$ mirror can tolerate this level of stress, we designed a launch constraint system that is predicted to expose no point on the mirror to greater than $300 \mathrm{psi}$ (Fig. 27). Also, if necessary, the launch constraint support structure could be used as a reaction structure for an active figure control system to mitigate G-release error.

\subsubsection{PM actuators}

The PM is attached to six hexapod actuators and 30 launch lock mechanisms (18 axial and 12 radial). The SM is also attached to six hexapod actuators and launch lock mechanisms.

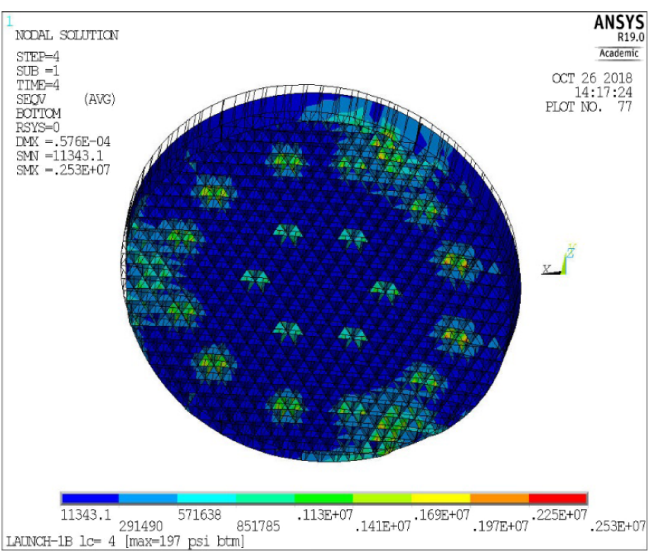

With launch locks

launch stress $=\sim 200 \mathrm{psi}$

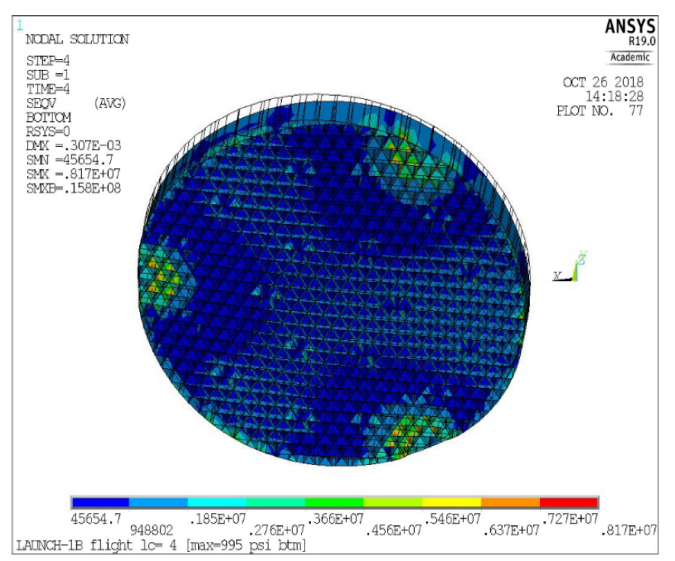

Without launch locks launch stress $=\sim 1000 \mathrm{psi}$

Fig. 27 Launch locks redistribute launch stress from the three hexapod attachment locations to the entire mirror. 


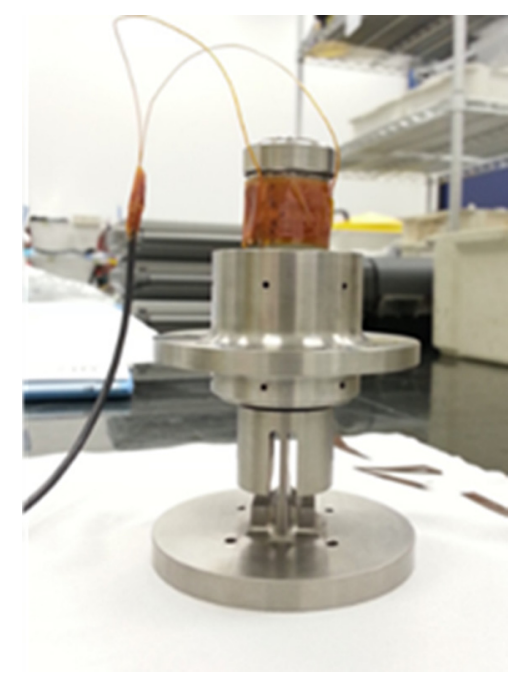

Fig. 28 AMTD fine stage (0.8-nm step) actuator.

These actuators and mechanisms are assumed TRL-9 components given that similar actuators and mechanisms are currently flying on Hubble and Kepler and will fly on JWST and WFIRST. For example, JWST uses a two-stage stepper-motor actuator for both launch restraint and alignment. Its coarse stage has a range of $20 \mathrm{~mm}$ (12.5 mm of which is used to deploy from the launch restraints). The fine stage uses a mechanical gear to drive an eccentric cam shaft with a step size of $7.7 \mathrm{~nm} .{ }^{59}$ Given that the fine stage is mechanical, smaller step sizes required for HabEx or Large UV/Optical/IR Surveyor (LUVOIR) can be achieved with a different gear ratio. In addition, the AMTD project designed, built, and characterized a fine-stage actuator with a range of $15 \mu \mathrm{m}$, step size of $0.8 \mathrm{~nm}$, mass of $0.313 \mathrm{~kg}$, and axial-stiffness of $41 \mathrm{~N} / \mu \mathrm{m}$ (Fig. 28). ${ }^{27}$ Figure 2 (0.8 nm step 8: AMTD fine stage) actuator. $^{27}$

\subsubsection{PM reflective coating}

The baseline reflectance coating for the PM, SM, and TM is a Hubble-like aluminum coating with a magnesium-fluoride protective overcoat. The coating and its deposition processes are TRL-9 and have been used in flight programs since the 1970s. HabEx has the same spectral range science requirement as Hubble.

\subsubsection{PM thermal control system}

The PM thermal control system is critical to the HabEx telescope's ability to achieve sciencerequired diffraction-limited performance and wavefront stability. The function of the thermal control system is to uniformly set the PM's front surface to the desired operating temperature and keep it at that temperature regardless of where the telescope points on the sky relative to the Sun. Control system accuracy impacts diffraction limit performance, signal-to-noise ratio, and spectral throughput. The precision with which the system can maintain temperature determines wavefront stability. Any gradient or bias error in the mirror's bulk temperature will introduce a static "cryo-deformation" WFE. And any temporal variation in the mirror's temperature will introduce instability.

Similar to Hubble, HabEx is cold biasing the PM and SM and heating them to the desired operating temperature. The desired operating temperature is constrained by two competing requirements. Near-IR science needs the mirrors to be cold to minimize in-field thermal noise. However, UV science requires the mirrors to be free of any contamination, such as a monolayer of water ice or other out-gassed molecules, to maximize spectral throughput. HabEx has selected an operating temperature of 260 to $270 \mathrm{~K}$ for its mirrors because it is above the sublimation temperature for water ice. The amount of cold bias is also constrained by competing requirements. When the bias is larger, it is easier to control the mirror temperature; however, the system 
will require more electrical power to achieve that control. The minimum amount of bias is one where the mirrors are cold biased for all potential Sun orientation angles. Cold bias is produced by thermal isolation around the telescope. Preliminary analysis required the removal of some spacecraft panels on the anti-Sun side to increase the cold bias.

Making the temperature of the PM's front surface uniform anywhere it points on the sky relative to the Sun is a bit more difficult. Because of the radiative transfer solid-angle view factor of the PM through the straylight tube to deep space, the middle of the mirror will be colder than the edge. Also, depending on the pointing angle to the Sun, the thermal load into the Sun-side of the telescope will change as a function of the angle cosine-thus changing the lateral gradient experienced by the PM. To compensate, the HabEx plans to use a thermal control system with radial and azimuthal heater zones behind and around the perimeter of the PM. The radial heater zones compensate for the view factor radial gradient to create a uniform front surface temperature. The azimuthal heater zones compensate for changes in the lateral thermal gradient as a function of pointing angle.

The baseline HabEx active radial thermal control concept is an engineering scale-up of systems built by the Harris Corporation. Zonal active thermal control of PMs is currently TRL-9 with systems currently flying on the Harris Corporation commercial 0.7- and 1.1-m Spaceview ${ }^{\mathrm{TM}}$ telescopes. These systems enable on-orbit focus adjustment for optimal image quality. ${ }^{60,61}$ In addition, under the NASA Astrophysics Division-funded PTCS, Harris Corporation has built and delivered to NASA for testing with candidate mirror assemblies a 1.5-m system with 37 thermal control zones (Fig. 29). ${ }^{62}$ This system has six azimuthal heater zones in each of five radial and circumferential zones. In addition, there are heater zones on each mirror strut and the support structure. The test article is fully instrumented to quantify radiative and conductive heat flow.

The ability to achieve the wavefront stability required for coronagraphy depends on the PM's thermal sensitivity and the thermal control system's controllability. Regarding thermal sensitivity, the rate at which the PM's SFE changes (i.e., stability) depends on its CTE, mass, and thermal capacity ${ }^{63}$

$$
\frac{\delta \mathrm{SFE}}{\delta t} \sim \frac{\mathrm{CTE}}{M c_{p}},
$$

where CTE is the coefficient of thermal expansion, $M$ is the mass, and $c_{p}$ is the specific heat. Other properties that influence response time are radiative emissivity and conduction to structural mass. But, to first order, when the mirror's mass is larger and its CTE is smaller, the mirror's response to thermal stimuli is smaller and slower. These are the reasons why monolithic mirrors fabricated from zero CTE materials such as ZERODUR ${ }^{\circledR}$ and $\mathrm{ULE}^{\circledR}$ glass are preferred.

The key to achieving an ultrastable thermal wavefront is to sense and correct fluctuations in the mirror's thermal environment faster than the mirror's response time to those changes. For any given mirror design, stability is achieved by a combination of two engineering specifications: control period and thermal fluctuation (sensor noise). For a generic mirror design, shown in Fig. 30, if the thermal sensors are uncertain to $50 \mathrm{mK}$, the control period needs to be about
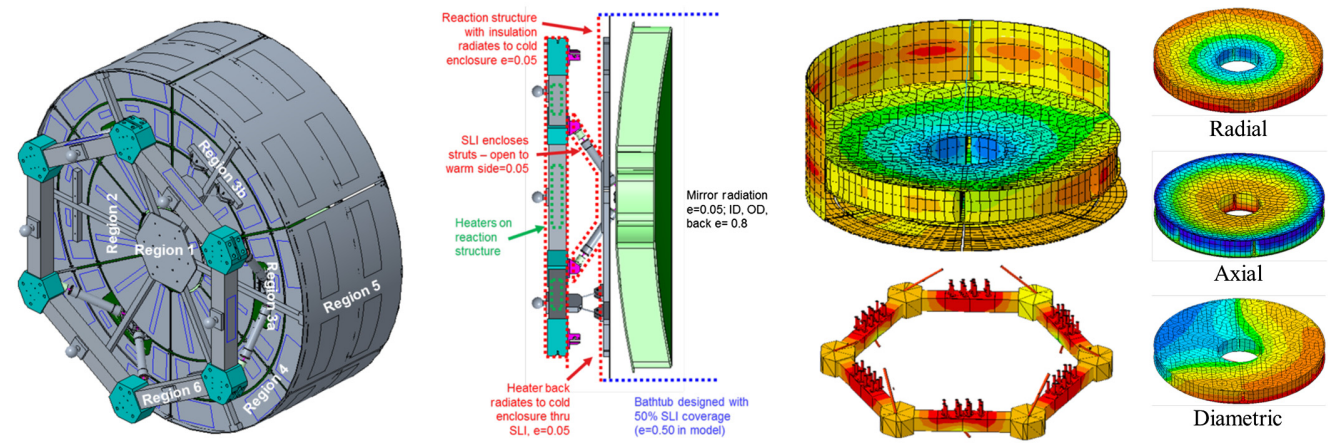

Fig. 29 Predictive thermal control study zonal thermal control system technology demonstrator. 


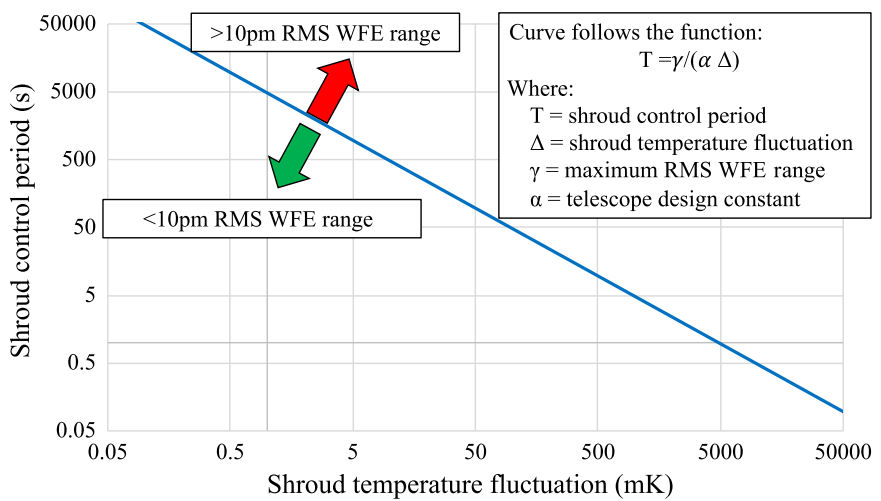

Fig. 30 Thermal wavefront stability is achieved by balancing thermal sensing noise and control period.

$50 \mathrm{~s}$. But, if the sensors are uncertain to $5 \mathrm{mK}$, the control period can be $500 \mathrm{~s}$. And a factor of $10 \times$ improvement is accomplished if the thermal system is controlled at $50 \mathrm{~s}$ with a 5 -mK noise sensor. The relationship is linear. As long as the thermal control system (period $\times$ fluctuation) product is below the mirror architecture tolerance line, the thermal control system will provide the specified wavefront stability. ${ }^{64}$

Given that thermal sensing system control periods can be on the order of a few seconds, the technical design parameter that determines wavefront stability performance is sensing noise. The WFE will be more stable when the sensing noise is lower. The current TRL-9 capability is defined by the Harris Corporation Spaceview ${ }^{\mathrm{TM}}$ telescope's thermal control systems sensors, which have a noise of $\sim 50 \mathrm{mK}$ and control their $1.1-\mathrm{m}$ telescope to a temperature of 100 to $200 \mathrm{mK} .^{65}$ It is important to note that these are Earth-viewing systems in low-Earth orbit. These same systems would be much more thermally stable if they were doing astrophysics science at SE-L2. STOP analysis presented in Sec. 4.3.2 shows that the more massive HabEx PM can be controlled to a temperature of $\sim 1 \mathrm{mK}$ with a system of $50 \mathrm{mK}$ noise and 30-s control period. Performance margin can be obtained using sensors with lower noise. The PTCS thermal enclosure system is using commercial platinum resistance thermometers with $\pm 5 \mathrm{mK}$ reproducibility and $\pm 10 \mathrm{mK}$ long-term stability. ${ }^{66}$ Currently, for WFIRST, Harris Corporation is designing a thermal control system that uses 4 -wire bridge-circuit thermistors with $<4 \mathrm{mK}$ noise. ${ }^{65}$

Finally, it is necessary to "tune" the thermal enclosure to "set" temperatures. Schott Corporation has the ability to "tailor" ZERODUR ${ }^{\circledR}$ to have 0 CTE at a specific temperature. However, the exact CTE at a specific temperature is uncertain by $\pm 10 \mathrm{ppb} / \mathrm{K}^{67}$ As shown in Fig. 20, the CTE/K slope at $\sim 270 \mathrm{~K}$ is $\sim 2.5 \mathrm{ppb} / \mathrm{K}$; thus, $\mathrm{a} \pm 10 \mathrm{ppb} / \mathrm{K}$ uncertainty translates into the actual $0 \mathrm{CTE}$ temperature being uncertain by $\pm 4 \mathrm{~K}$. Therefore, it may be necessary to adjust the on-orbit temperature of the PM and SM thermal enclosures a few $\mathrm{K}$ to optimize wavefront stability. In addition, zonal temperature tuning can be accomplished to compensate for CTE inhomogeneity.

\subsection{SM Assembly}

The SMA is an integrated optomechanical system consisting of a mirror substrate, mount, support structure, launch lock system, and thermal control system. The mirror substrate is a $0.45-\mathrm{m}$ diameter, off-axis ZERODUR ${ }^{\circledR}$ mirror. ZERODUR ${ }^{\circledR}$ was selected based on its expected CTE homogeneity. The SMA mount, support structure, launch locks, and thermal control system are similar to those of the PMA. Because the TM is fixed, the SMA is actuated via a hexapod to maintain its optical alignment with the PM and TM. The SMA and supporting laser-truss system (not shown) are mounted to the top of the tower structure (Fig. 31).

Per Sec. 2.1, to achieve 400-nm diffraction-limited performance, the SMA is assigned a WFE of $15 \mathrm{~nm}$ rms (surface error of $7.5 \mathrm{~nm} \mathrm{rms}$ ). For a $0.45-\mathrm{m}$ size mirror, a $<7-\mathrm{nm}$ rms SFE is easily achievable. The AMTD study demonstrated $5.4 \mathrm{~nm}$ rms on a $0.43-\mathrm{m}$ mirror with a $10-\mathrm{mm}$ facesheet. ${ }^{28}$ Furthermore, because the SLS has significant mass margin, the SM can have a 


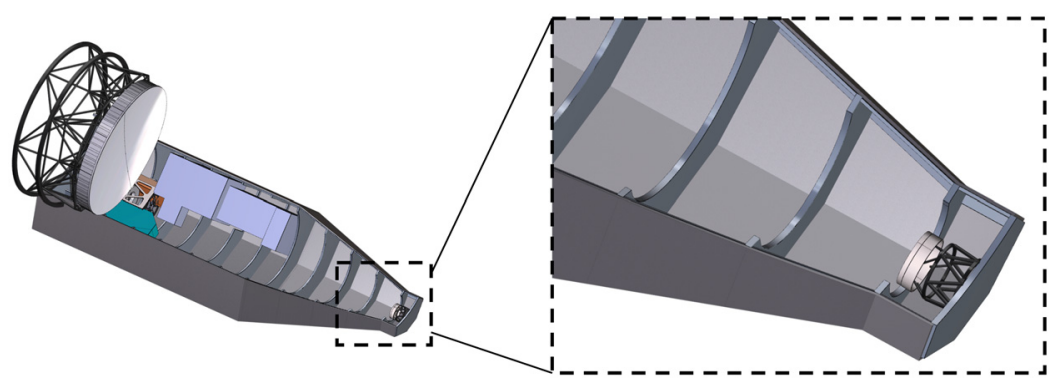

Fig. 31 SMA mounted to top of tower structure.
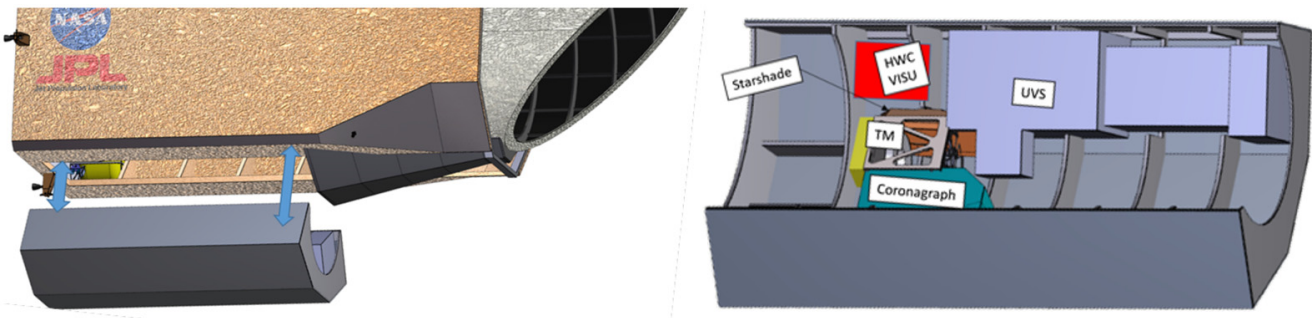

Fig. 32 Serviceable ISIM.

thicker facesheet to minimize mid-spatial frequency error (which is important for coronagraphy). It is very likely that the $\mathrm{SM}$ total surface error could be $<5 \mathrm{~nm} \mathrm{rms}$. The design, manufacture, and verification of the SMA and its constituent components are considered to be well within the state of practice for space telescopes.

\subsection{Integrated Science Instrument Module}

The ISIM is an optomechanical structure whose function is to maintain optical alignment of the science instruments relative to the TM, including the coronagraph, starshade instrument, UV spectrograph, workhorse camera, and fine guidance system. This is particularly important because each science instrument uses a different portion of the main toroidal TM (the ultraviolet spectrograph uses a separate on-axis TM). In the HabEx TMA design, the TM's location is fixed, and the PM and SM are aligned to the TM. The ISIM structure is also a key component of the overall tower structure. The ISIM is a true integrated module. It is designed to be removed from the observatory on precision Hubble Space Telescope (HST) style optical rails as a whole module for servicing (Fig. 32). Or, once removed, individual science instruments can be replacedagain using precision HST style optical rails.

\section{Telescope Structural Thermal Optomechanical Performance}

As discussed in Sec. 2, to accomplish the HabEx science goals requires a 400-nm diffractionlimited telescope with an ultrastable wavefront. And, as discussed in Sec. 3, an iterative process was used to design a mechanically stiff and thermally stable telescope architecture. This iterative design process was guided by STOP modeling. The result is a baseline telescope that meets its required performance specifications.

\subsection{Integrated Model}

To evaluate whether the baseline HabEx telescope meets its performance requirements, integrated thermal and finite element models (FEM) of the telescope and spacecraft bus were constructed and then merged into an observatory model. An observatory-level model was required 
Stahl et al.: Habitable-Zone Exoplanet Observatory baseline 4-m telescope...

Table 7 Integrated model details.

\begin{tabular}{lcc}
\hline \hline Element & Telescope FEM & Spacecraft FEM \\
\hline Degrees of freedom & 221,658 & 282,390 \\
Number of elements & 42,953 & 57,813 \\
Element types & $\begin{array}{c}\text { CQUAD4, CTRIA3, CBAR, } \\
\text { CBUSH, CONM2 }\end{array}$ & $\begin{array}{c}\text { CQUAD4, CTRIA3, CBAR, } \\
\text { CBUSH, CONM2 }\end{array}$ \\
Multipoint constraints & 426 & 64 \\
Number of grids & 36,928 & 47,065 \\
\hline \hline
\end{tabular}

because the telescope's thermal and mechanical performance is strongly influenced by the spacecraft. These models were used to perform STOP analyses.

NASTRAN solution sequences were used for static and dynamic mechanical analysis of the integrated observatory: solution 101 (linear statics) for strength checks, solution 103 for normal modes analysis, solution 105 for buckling/stability analysis, solution 111 for LOS stability analysis, and solution 112 for transient ring-down analysis. Thermal Desktop and SigFit were used for thermal analysis. Thermal mapping to the FEM was calculated via Thermal Desktop and applied as loads to the NASTRAN model using solution 101 to predict thermal deformations.

\subsubsection{Mechanical model}

The integrated observatory FEM was created using the MSC Patran, preprocessor, and geometry created in ProEngineer computer aided design (CAD). The PM and SM FEMs were created independently using the NASA MSFC-developed Arnold Mirror Modeler. Using the integrated NASTRAN model, analyses were performed to ensure strength/stability and stiffness requirements were satisfied in accordance with NASA-STD5001B and the launch vehicle payload users guide (United Launch Alliance, ULA-Delta IV Heavy). In addition, the integrated FEM was used to perform dynamic response and thermal analysis. Table 7 summarizes the models.

Structural elements utilize composite construction where possible to provide a rigid and lightweight design. Where possible, M55J carbon composite material is used due to its excellent strength/stiffness and low mass-density $\left(1.58 \mathrm{~g} / \mathrm{cm}^{3}\right)$ specifications. Telescope structure skins, circumferential ribs, axial webs, and the forward contamination door utilize Honeycomb Sandwich Construction with M55J facesheets with Hexcel honeycomb core. Mirror support truss members assume M55J circular tube construction with titanium end fittings. The M55J unidirectional composite layup orientations were tailored for maximum performance and minimum mass. Structural damping is specified to be $0.0005(0.05 \%)$.

\subsubsection{Thermal model}

The integrated observatory thermal model was created in Thermal Desktop using the geometry created in Pro-Engineer CAD. The Thermal Desktop model has $20 \mathrm{~K}$ elements and calculates the telescope's structure and mirror temperature distribution at $10 \mathrm{~K}$ nodes. The temperature distribution for each node is mapped onto the NASTRAN FEM, and the deflections created by each node's CTE are calculated using NASTRAN solution 101. RBM and mirror surface deformations are calculated from the NASTRAN deflections using SigFit. During the analysis stage, the calculated RBMs were replaced by a residual error to simulate correction via the laser metrology system. The PM and SM's mesh grids were sized to enable SigFit to fit thermally induced SFE to higher-order Zernike polynomials.

The model assumes multilayer insulation (MLI) to control heat loss and to isolate thermal disturbances (i.e., the Sun). Radiators pull heat from the science instruments and spacecraft electronics. Between the MLI and radiators, the payload is passively cold biased. Active thermal enclosures are used to maintain the operating temperature of the PM and SM at $\sim 270 \mathrm{~K}$

J. Astron. Telesc. Instrum. Syst. $\quad$ 034004-26 Jul-Sep 2020 • Vol. 6(3) 
Table 8 Thermal model details.

\begin{tabular}{lc}
\hline \hline Proportional control zones & 82 \\
\hline PM thermal enclosure & 6 \\
PM truss hexapod legs & 9 \\
SM thermal enclosure & \\
Bang-bang survival heater zones & 18 \\
Telescope baffle tube & 7 \\
Telescope secondary tower & 3 \\
Spacecraft bus structure & 8 \\
\hline Spacecraft fuel tanks
\end{tabular}

(see Sec. 3.3.7). Without these heaters, the model predicts a PM temperature of $206 \mathrm{~K}$ and an SM temperature of $196 \mathrm{~K}$. The model assumes TRL-9 capabilities for the enclosure specifications: thermal sensors with $50-\mathrm{mK}$ measurement uncertainty and proportional controller systems (proportional integral derivative, PID) operating with 30-s periods. The model has a total of 133 control zones. Of these, 36 are bangbang survival heaters set at $212 \mathrm{~K}$ and 97 are PID control zones (Table 8). They are set to keep the PM and SM front face temperatures at $\sim 270 \mathrm{~K}$. The model predicts that the PM front surface will have $~ 200-\mathrm{mK}$ "trefoil" gradient (Fig. 33). The source of this gradient is thermal conduction into the hexapod struts. The mirror will have $\sim 3 \mathrm{~K}$ front to back gradient caused by radiative coupling to deep space as well.

The PM and SM CTE are modeled as consisting of a uniform "bulk" CTE and a CTE homogeneity distribution. The uniform CTE value determines the mirror's low-order shape response to bulk temperature changes and/or gradient temperature changes (i.e., axial, radial, or lateral). Such temperature changes can produce low-order errors such as power and astigmatism. The homogeneity distribution determines the mirror's mid-spatial response. The model calculates mirror shape changes from two effects: response of mirror with uniform CTE to changes in

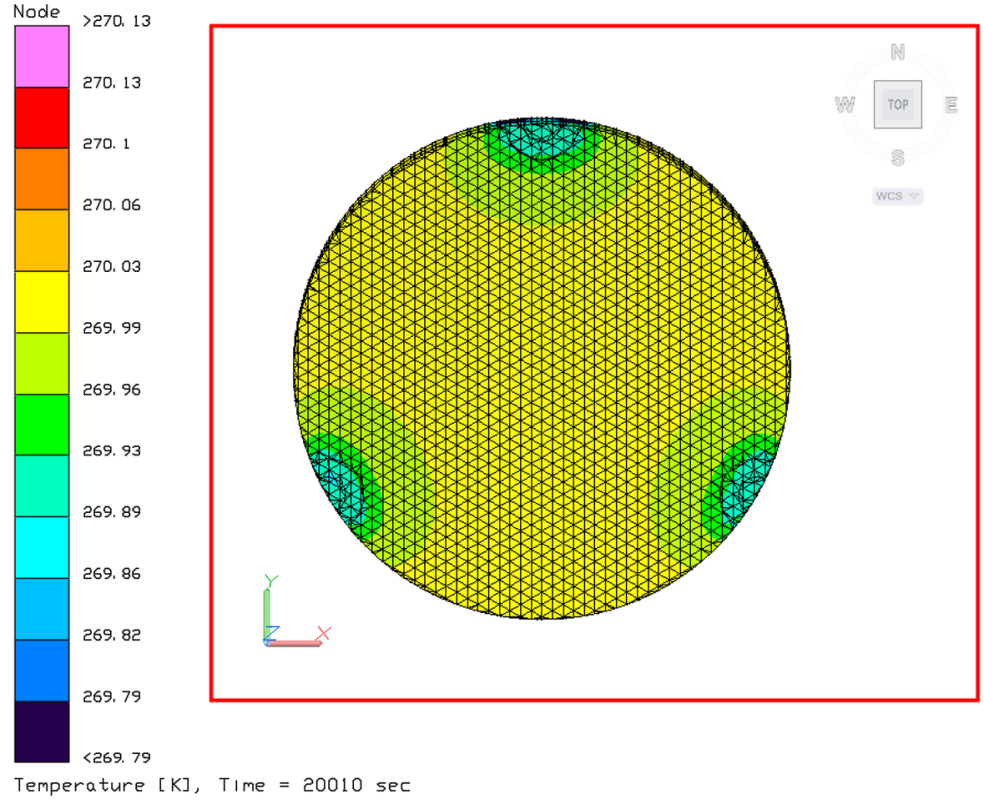

Fig. 33 Predicted 200-mK trefoil thermal distribution of PM front surface. 


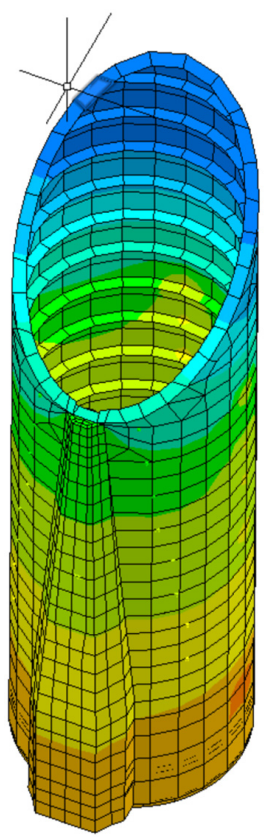

(a)

\section{(3)}

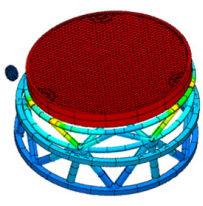

(b)

Fig. 34 On-orbit temperature of (a) baseline telescope structure baffle tube and (b) PMA.

temperature at each of the 10-K nodes and response of a mirror with a CTE inhomogeneity distribution to a uniform bulk temperature change.

Per Fig. 20, Schott Corp. has the ability to tailor ZERODUR ${ }^{\circledR}$ to have a 0 CTE at $275 \mathrm{~K}$ $\left(-18^{\circ} \mathrm{C}\right)$. The uncertainty of Schott's ability to achieve $0 \mathrm{CTE}$ at $275 \mathrm{~K}$ is $\pm 10 \mathrm{ppb} / \mathrm{K} .^{67}$ Therefore, the model assumes a uniform bulk CTE of $20 \mathrm{ppb} / \mathrm{K}$. One method to estimate CTE inhomogeneity is to measure the thermal deformation of the mirror and assume that CTE is linear with temperature. As part of the AMTD project, a 1.2-m ELZM was measured to have an $\sim 11$-nm-rms deformation over a 62-K thermal range (from 292 to $230 \mathrm{~K}$ ). Figure 6 shows the measured error and its decomposition into Zernike polynomials. ${ }^{13}$ The model assumes this measured thermal signature for its CTE inhomogeneity distribution. Obviously, the final flight mirror substrate must be selected based on measured CTE homogeneity. To first order, CTE homogeneity data can provide an initial estimate of the required mirror thermal stability. For example, if a $60 \mathrm{~K}$ thermal change produces $5 \mathrm{~nm}$ rms of trefoil wavefront and the error budget tolerance is $0.5 \mathrm{pm} \mathrm{rms}$, then the mirror needs to be kept thermally stable to $6 \mathrm{mK}$.

The primary weakness of the current thermal design is the structure, which is very simple. It assumes that the PM support truss structure, telescope tower, and tube are uniformly composed of TRL-9 composite material M46J with $-334.2 \mathrm{ppb} / \mathrm{K}$. There are no elements to represent adhesive joints, and there has been no effort to athermalize the structure by application of positive/negative CTE materials. As a result, the telescope model does not accurately model structural thermoelastic effects. In addition, the current design does not actively control the structure temperature. Consequently, the model is highly sensitive to thermal changes. The justification for this design/analysis decision is that the telescope will have a laser truss system to track slow moving thermal structural changes. As shown in Fig. 34, the thermal model predicts that, without any heaters, the tube will have a gradient of over $100 \mathrm{~K}$, and the PM truss will have an $\sim 20-\mathrm{K}$ gradient. As discussed in Sec. 4.2.1, these gradients will produce $<0.5 \mathrm{~mm}$ of mechanical displacement — which can be easily corrected by the actuators, discussed above in Sec. 3.3.5.

\subsubsection{Mechanical analysis input}

Dynamic mechanical errors (LOS jitter, LOS WFE stability, inertial WFE stability, and impulse ring-down) are caused by structural response to mechanical stimuli. To minimize the source of such stimuli, the baseline HabEx observatory architecture does not use reaction wheels for 


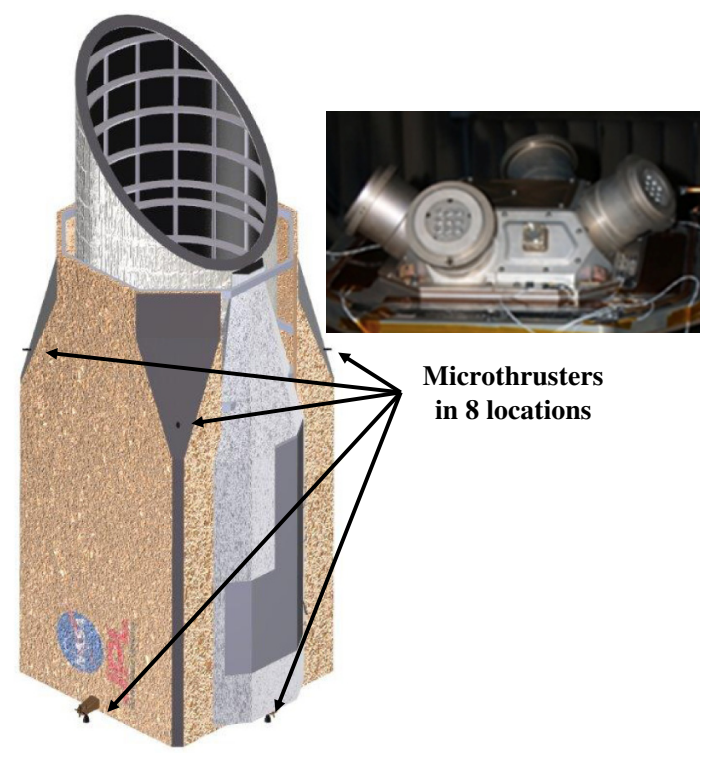

Fig. 35 Microthruster locations.

slewing or pointing. Instead, it uses thrusters and microthrusters. Thrusters, located at the base of the telescope, slew and point the telescope. They are then turned off and microthrusters maintain pointing for the duration of a science exposure. The microthruster noise is the only source of mechanical stimuli. Both thrusters are attached to the spacecraft, and their transmission path to the telescope is via the interface ring. If necessary, this ring can be isolated from the telescope. As shown in Fig. 35, there are four microthruster modules located at the base of the observatory (aft) and another four modules located above the center of solar pressure (forward). Each forward module consists of four heads, each with nine emitters, covering a 90-deg cone. Each aft module consists of four heads, each with 18 emitters.

Microthrusters run continuously with thrust proportional to applied current. Figure 36 shows a measured noise PSD for a single colloidal microthruster emitter. The data indicate that microthruster emitters have a maximum noise of $0.03 \mu \mathrm{N} / \sqrt{\mathrm{Hz}}$ with a roll-off after about $2 \mathrm{~Hz}^{68}$ To be conservative, because the data are noisy and have not been measured beyond $5 \mathrm{~Hz}$,

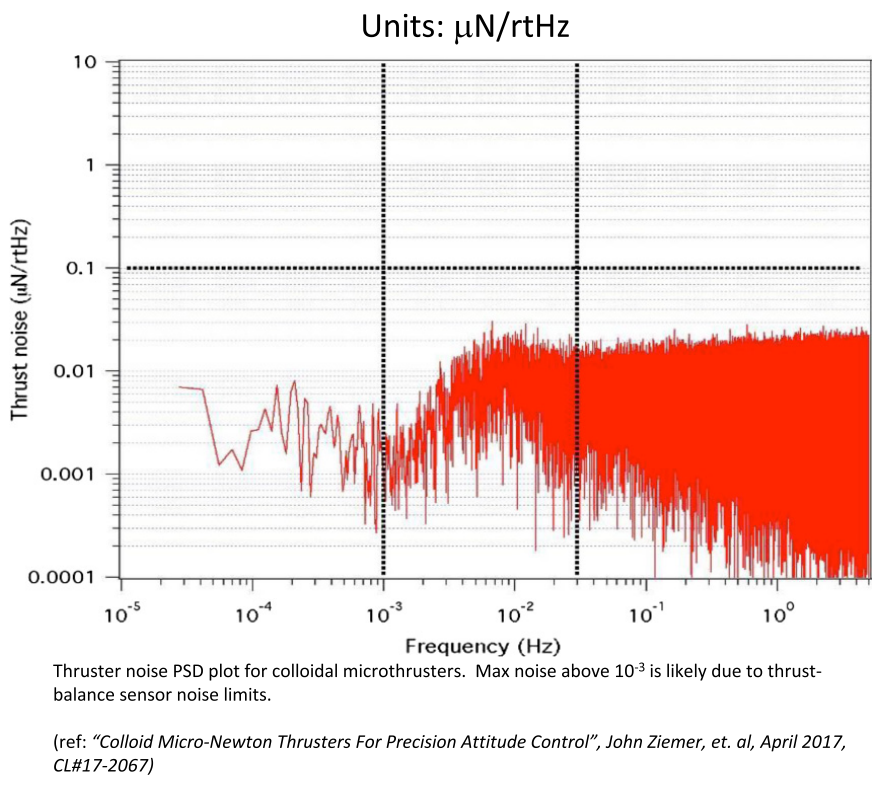

Fig. 36 PSD noise plot for colloidal micro-thrusters. 
the HabEx STOP analysis assumed that each microthruster emitter has a flat or "white" noise spectrum of $0.1 \mu \mathrm{N}$-providing at least a $3 \times$ specification margin. Note that this noise profile is identical to the specification used by LISA and ST-7. ${ }^{68}$ Finally, because the aft modules have twice as many emitter heads as the forward modules, each forward module is specified to have a flat noise spectrum of $0.4 \mu \mathrm{N} / \sqrt{\mathrm{Hz}}$ and each aft module is specified to have $0.8 \mu \mathrm{N} / \sqrt{\mathrm{Hz}}$.

Finally, because the noise "forcing-function" amplitudes are extremely small, values reported by the analysis could have significant uncertainty. While the FEM's predicted performance is linear as a function of input, the physical system being modeled may not be linear. To mitigate this risk, a model uncertainty factor (MUF) is used in some analyses, while in others an MUF is not applied. For each analysis, the MUF status is made explicit.

\subsubsection{Thermal analysis input}

Dynamic thermal errors (LOS drift, LOS WFE, and thermal WFE stability) are caused by structural and optical component response to thermal stimuli. To predict the telescope's thermal performance at SE-L2, DRMs were analyzed in Thermal Desktop. The calculated temperature distribution is mapped onto the NASTRAN FEM and the deflections of each node calculated. RBMs and SFE of the PM and SM were calculated from the NASTRAN deflections using SigFit.

The DRM starts by pointing the telescope at a reference star to dig the dark hole. The analysis assumes that the telescope reaches a steady-state thermal condition at this Sun orientation. Next, the telescope is pointed at the science star. To make the analysis worst-case, it is assumed that this requires a $+\theta$ - deg pitch away from the Sun (Fig. 37). To facilitate speckle subtraction, the telescope is rolled $\pm \Phi$ deg about the science star vector (Fig. 38).

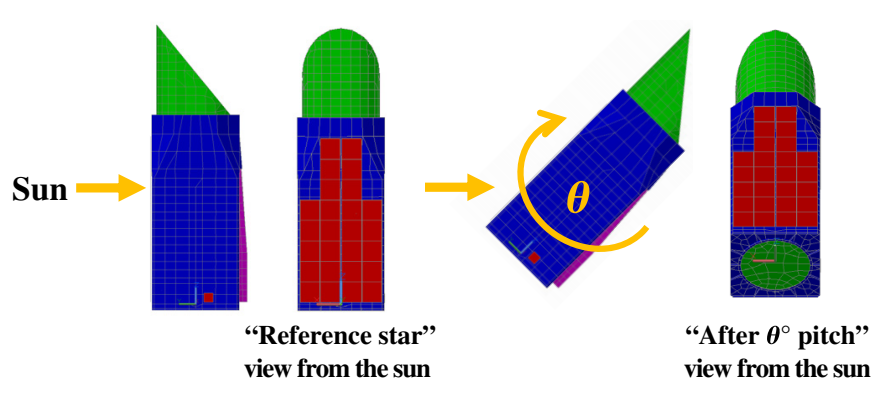

Fig. 37 Nominal observing scenario slews for thermal analysis.
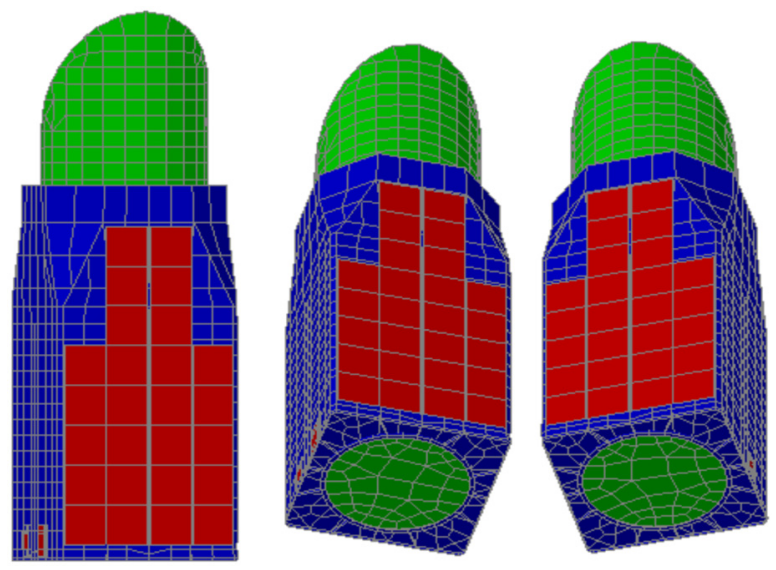

Fig. 38 Telescope motions as viewed from the Sun. 


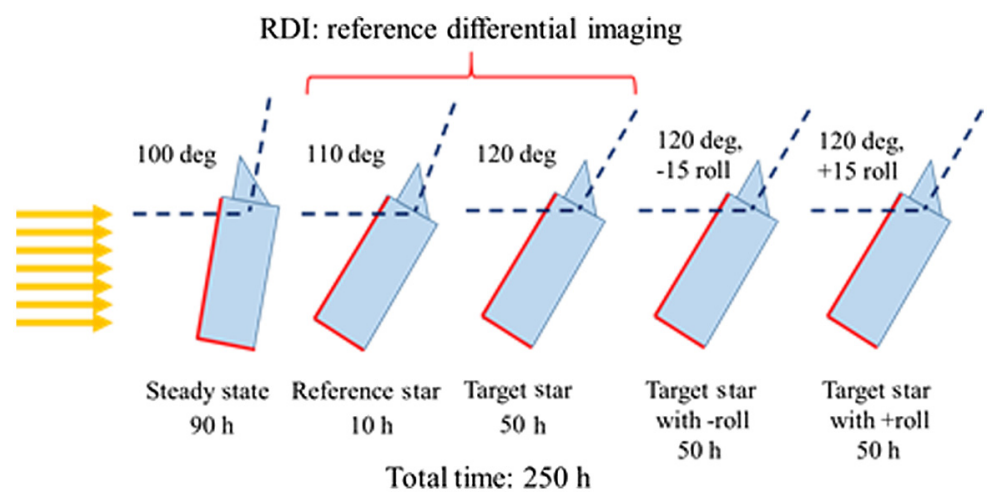

Fig. 39 Analyzed DRM.

The specified DRM analyzed is (Fig. 39):

- $T=0$-h drop into SEL2 with observatory pointing 100 deg to Sun

- $T=90-\mathrm{h}$ pitch +10 deg away from Sun to Reference Star \& 'Dig' Dark Hole

- $T=100-\mathrm{h}$ pitch +10 deg to Science Star

- $T=150$-h roll $-15 \mathrm{deg}$

- $T=200-\mathrm{h}$ roll $+30 \mathrm{deg}$

- $T=250-\mathrm{h}$ end science observation

The STOP Analysis calculates as a function of DRM (time):

- Temperature of PM and SM

- WFE produced by PM and SM temp changes

- RBM between PM and SM

- WFE produced by noncorrectable RBM (i.e., after laser truss)

- Total telescope WFE stability

Figure 40 shows how well the modeled active zonal thermal enclosure controls the temperature of the PM. Once the science observation begins, the PM temperature only changes by $\pm 0.15 \mathrm{mK}$. The main reason for this stability is the PM's thermal mass. The thermal time constant of the PM is $\sim 20 \mathrm{~h}$. Note that roll maneuvers produce negligible thermal error. Also note that, if the science star and reference stars were located such that the Sun angle remained unchanged during the slew (i.e., rotate around the sun vector), then there would be no change to the telescope's thermal environment.

\subsection{Diffraction-Limited Performance}

Diffraction-limited performance is important for both general astrophysics and coronagraphy because it (along with aperture diameter) determines the observatory's PSF. The wavelength

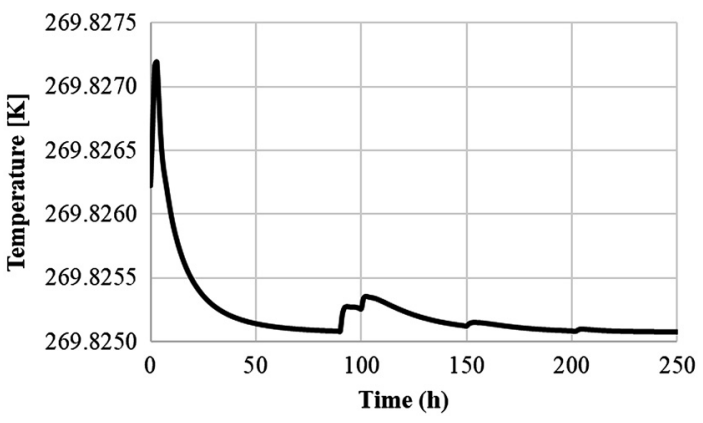

Fig. 40 250-hour DRM average PM temperature with PID control for $270 \mathrm{~K}$. 
of diffraction limited performance (WDLP) is determined by the observatory's rms transmitted WFE. Typically, WDLP is $\sim 13$ times rms WFE. Thus, for a 400-nm WDLP telescope, transmitted WFE must be $\sim 30 \mathrm{~nm}$ rms. Figure 4 presents a potential flow-down error budget of the observatory level WFE to the telescope. Assuming that the telescope mirrors are made to their optical prescription, the biggest potential WFE source is the ability to align the mirrors and maintain that alignment on-orbit. Table 2 illustrates a potential flow-down of the telescope error budget to the PM. Potentially, the biggest error source in achieving this specification is the ability to quantify and back-out gravity effects (i.e., a G-release error).

\subsubsection{On-orbit optical alignment}

Space telescopes are not launched in their final optical alignment. To survive launch, mirrors are retracted against launch restraints. They are then deployed and aligned on-orbit. For example, the JWST PMSAs are deployed $12.5 \mathrm{~mm}$ from their launch restraints to their operational position. ${ }^{59}$ In addition, space telescopes are not launched in their thermal state. Space telescopes are launched "warm," nominally $300 \mathrm{~K}$, and then cooled to their operational temperature. This thermal shift can change the shape of both the mirrors and the structure. For example, because the baseline HabEx structure design has not been athermalized, the integrated thermal model predicts that structure changes will result in rigid body displacements for the PM of 250 to $500 \mu \mathrm{m}$, 25 to $50 \mu \mathrm{m}$ for the SM, and rotations on the order of 20 to $50 \mu$-radian for both.

Thus, an on-orbit system is required to establish and maintain alignment between the PM, SM, and TM. To sense these thermodeformation errors, the baseline telescope architecture has a laser metrology system. And, to correct these errors, the baseline telescope architecture has hexapod actuator systems for the PM and SM with a 20-mm range and subnanometer resolution (Sec. 3.3.5). Assuming that the individual laser gauges have a $0.1-\mathrm{nm}$ rms measurement uncertainty, the alignment system is predicted to have a positional uncertainty (Table 9) sufficient to align the telescope to an LOS accuracy of $<0.2$ mas (on-sky) with a wavefront residual error of $<10 \mathrm{pm}$ rms.

\subsubsection{PM G-release error}

As discussed in Sec. 2.1.3, G-release error is a significant risk to achieving a 400-nm diffractionlimited telescope. Section 3.3.3 discusses how the HabEx PM is specifically designed to minimize the risk of G-release error by making the mirror stiff. Figure 23 shows that the baseline ZERODUR $^{\circledR}$ mirror has a predicted $1 \mathrm{G} X, Y, Z$ gravity sag of $(18.6,18.4$, and $12.6 \mu \mathrm{m})$ RMS. And, as also discussed in Sec. 3.3.3, while methods exist to characterize self-weight deflection and produce $0-\mathrm{G}$ space mirrors, it is also possible to mitigate G-release error risk via an active mirror (PM, SM, or DM). For example, analysis of the alternative ULE ${ }^{\circledR}$ HabEx PM indicates that 15 actuators can reduce G-release error by $20 \times, 25$ can reduce G-release error by $40 \times$, and 50 actuators can reduce G-release error by $100 \times$ (Fig. 41$).{ }^{70}$

\subsection{Predicted LOS Stability Performance}

LOS instability is important because it causes WFE and PSF smearing that degrades spatial resolution and IWA. LOS instability is caused by RBM of the optical components relative to each other. RBM can occur at the component level or can be caused by flexible-body effects of the structure. They are traceable to two sources: thermal and mechanical. Thermal LOS drift

Table 9 Laser metrology system positional uncertainty relative to the TM. ${ }^{69}$

\begin{tabular}{lcccccc}
\hline \hline DOF & $\Delta x(\mathrm{~nm})$ & $\Delta y(\mathrm{~nm})$ & $\Delta z(\mathrm{~nm})$ & $\Theta x(\mathrm{nrad})$ & $\Theta y(\mathrm{nrad})$ & $\Theta z(\mathrm{nrad})$ \\
\hline Primary & 2.117 & 2.129 & 0.278 & 0.240 & 0.272 & 1.068 \\
Secondary & 0.997 & 0.989 & 0.130 & 0.189 & 0.523 & 3.823 \\
Tertiary & 0 & 0 & 0 & 0 & 0 & 0 \\
\hline \hline
\end{tabular}




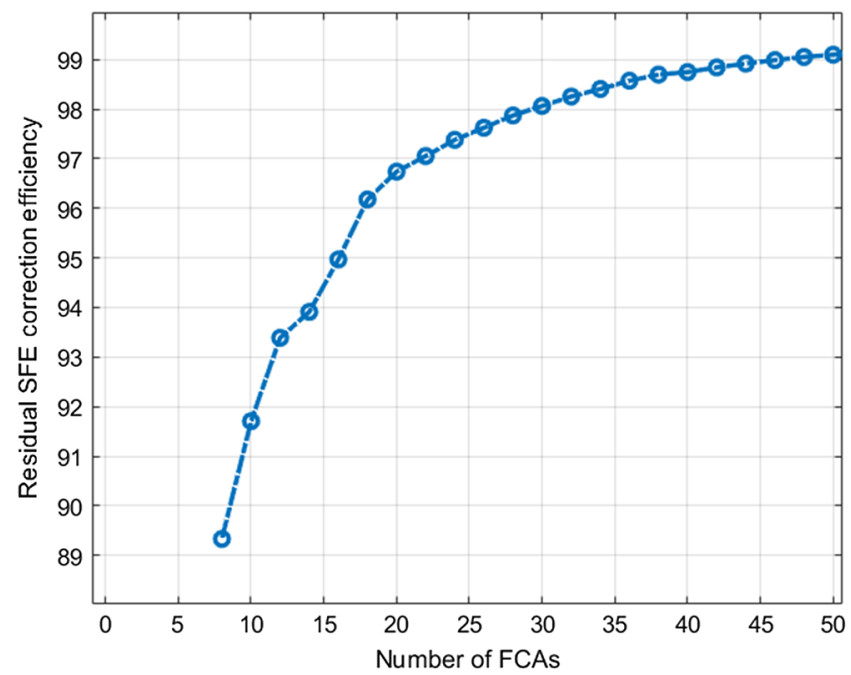

Fig. 41 Correctability of alternative ULE ${ }^{\circledR}$ 4-m PM design as a function of actuators.

occurs when the telescope is slewed relative to the Sun. Temperature change causes the telescope structure to expand or contract. Mechanical LOS jitter is produced by mechanical disturbance accelerations (from sources such as reaction wheels, cryo-coolers, etc.) exciting modes in the telescope structure. Typically, thermal LOS drift is slow and mechanical LOS jitter is fast. Per Sec. 2.2, the HabEx is baselining a ZWFS that can sense and correct LOS instability on the order of 2.5 mas at frequencies below $10 \mathrm{~Hz}$. In addition, HabEx is baselining a laser truss system to correct for slow thermal drift. Thus, per Table 3, the HabEx telescope LOS is specified to be stable to $<2$ mas for frequencies slower than $10 \mathrm{~Hz}$ and $<0.3$ mas for frequencies faster than $10 \mathrm{~Hz}$. Figure 7 provides one realization of the 0.3 mas specification.

To calculate whether the baseline telescope meets the specification, NASTRAN multipoint constraint equations created by SigFit were used to determine rigid body displacements of the PM and SM, relative to the TM (Fig. 42). STOP analysis was performed with the integrated FEM and thermal models to calculate the RBMs of the PM, SM, and TM caused by mechanical disturbance (Sec. 4.2.1) and thermal drift (Sec. 4.2.2).

\subsubsection{Predicted mechanical LOS stability performance}

To predict mechanical LOS jitter, the rigid-body motion of each mirror was calculated as a result of the structure's response from 0 to $350 \mathrm{~Hz}$ to the microthruster noise specification applied to the structure from 0 to $10 \mathrm{~Hz}$. Per Fig. 36, the microthruster specification provides at least

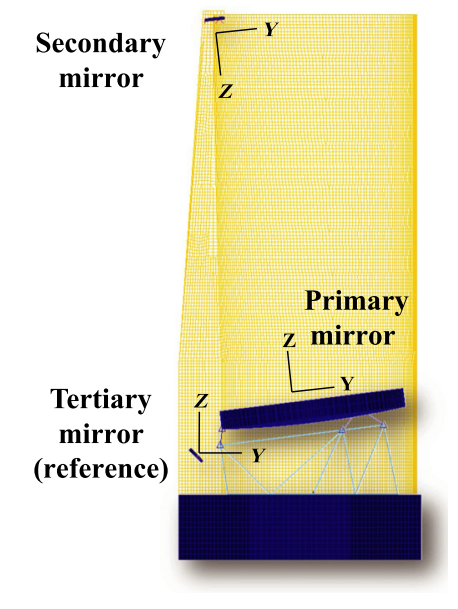

Fig. 42 Mirror displacements are relative to TM. 
Stahl et al.: Habitable-Zone Exoplanet Observatory baseline 4-m telescope...
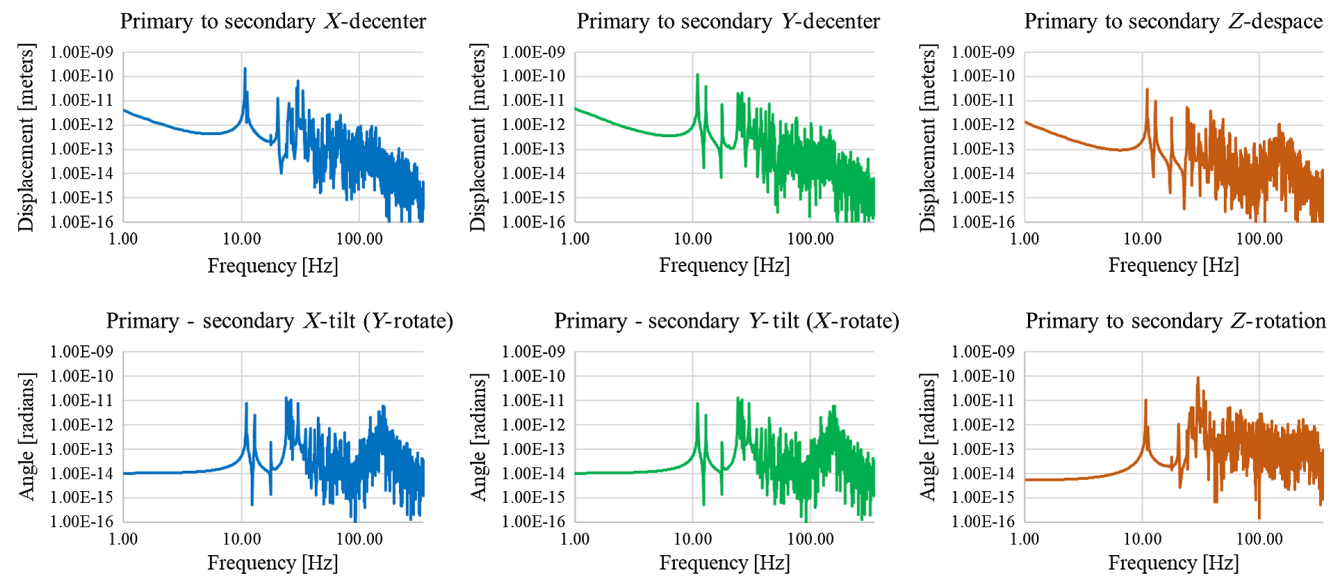

Fig. 43 PM-to-SM rigid body amplitudes for microthruster noise specification.

a $3 \times$ margin at low frequencies and more margin at higher frequencies-because the flat specification ignores mass damping-relative to the anticipated microthruster performance. In addition, an MUF of 2 was applied for a total margin of $\sim 4 \times$. Figure 43 shows the predicted displacement for the PM relative to the SM as a function of frequency. This presentation format is typical for an observatory that uses reaction wheels, because the wheels excite each mode as their speed changes. But microthrusters excite all modes simultaneously. Therefore, it is necessary to rss the individual components into a running sum. Figures 44 and 45 show the cumulative rigid body displacement and rotations for the PM and SM.

Table 10 lists the maximum predicted amplitude for each RBM. These motions predict an onsky LOS jitter of 0.03 mas, which provides a $\sim 10 \times$ margin against the 0.3 -mas specification. This stability implies that the baseline telescope does not need a low-order, ZWFS-controlled, fast-steering mirror.

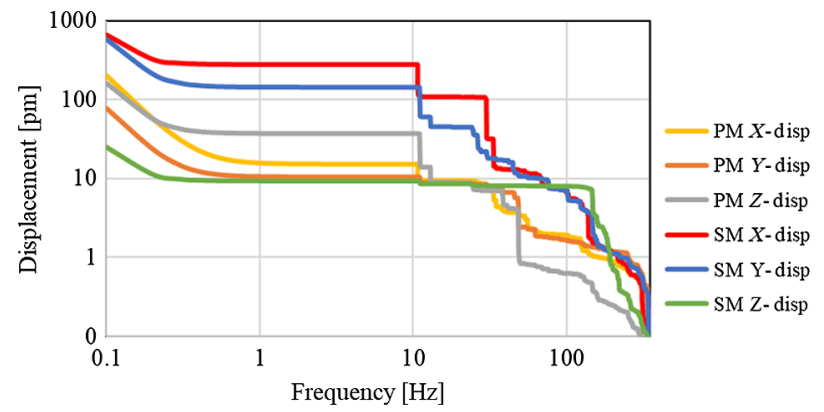

Fig. 44 Cumulative PM and SM rigid body displacement for microthruster noise specification.

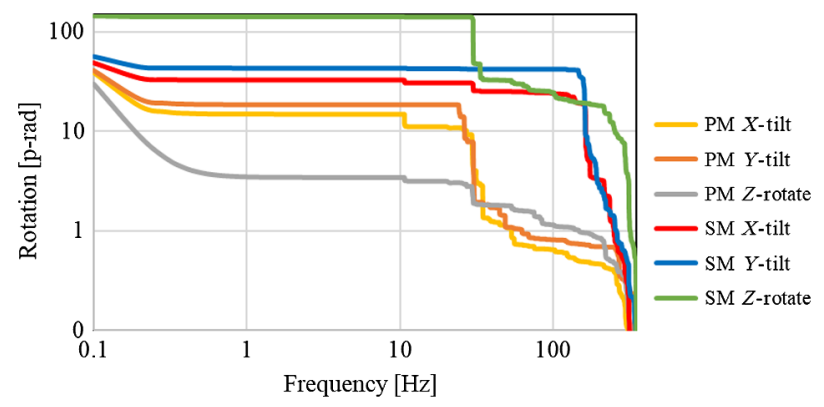

Fig. 45 Cumulative PM and SM rigid body rotations for microthruster noise specification. 
Table 10 Predicted maximum "jitter" motion of PM and SM from microthruster noise.

\begin{tabular}{lcccccc}
\hline \hline DOF & $\Delta x(\mathrm{~nm})$ & $\Delta y(\mathrm{~nm})$ & $\Delta z(\mathrm{~nm})$ & $\Theta x(\mathrm{nrad})$ & $\Theta y(\mathrm{nrad})$ & $\Theta z(\mathrm{nrad})$ \\
\hline Primary & 0.2 & 0.08 & 0.16 & 0.04 & 0.04 & 0.03 \\
Secondary & 0.67 & 0.58 & 0.03 & 0.05 & 0.06 & 0.15 \\
\hline \hline
\end{tabular}

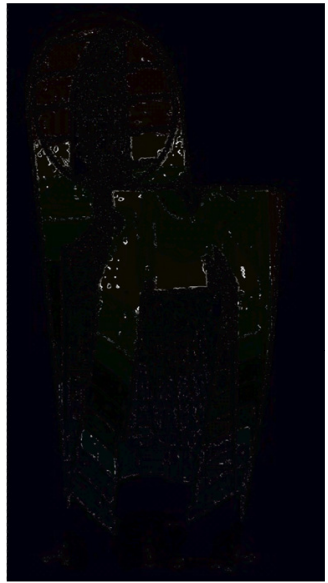

$11 \mathrm{HZ}$ twist mode

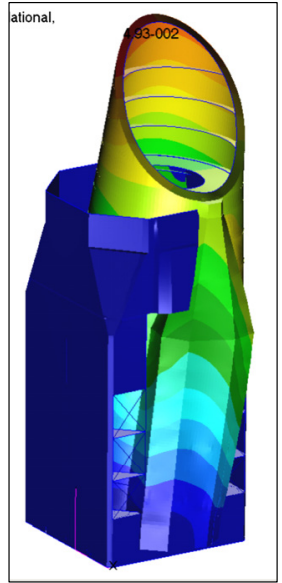

$12.8 \mathrm{HZ}$ bend mode

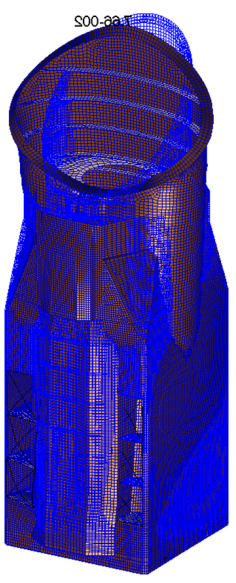

24.7 HZ flex mode

Fig. 46 Tube modes.

Figures 44 and 45 show information regarding frequencies of various structural modes and the coupling of those modes with optical components. For example, the displacements that occur at $11 \mathrm{~Hz}$ are associated with a twisting mode between the spacecraft and telescope (Fig. 46). There is a tube-bending mode at $12.8 \mathrm{~Hz}$ and a flexing mode at $24.7 \mathrm{~Hz}$. The $30-\mathrm{Hz}$ mode is bending between the SM tower and PM truss structure. Moreover, the displacements in the 45- to $50-\mathrm{Hz}$ range are caused by motions of the PM on its mount (Figs. 24 and 25).

\subsubsection{Predicted thermal LOS stability}

To predict thermal LOS drift, the rigid-body motions of each mirror were calculated as a result of the 250-h DRM discussed in Sec. 4.1.4. Figures 47 and 48 show the $X, Y, Z$ rigid body residual displacements of the PM and SM (i.e., the amount of thermal RBM that is not corrected by the laser metrology system that senses and controls optical alignment of the PM and SM).

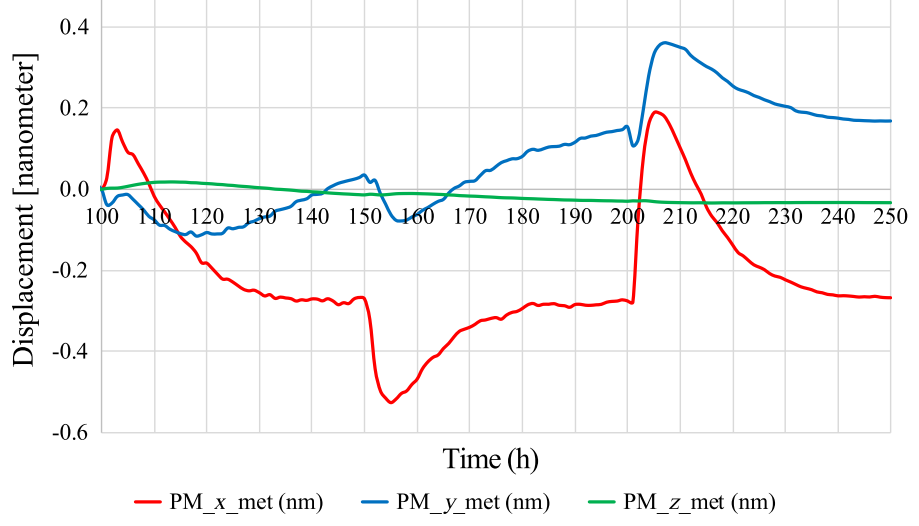

Fig. 47 Displacements of PM due to thermal DRM. 


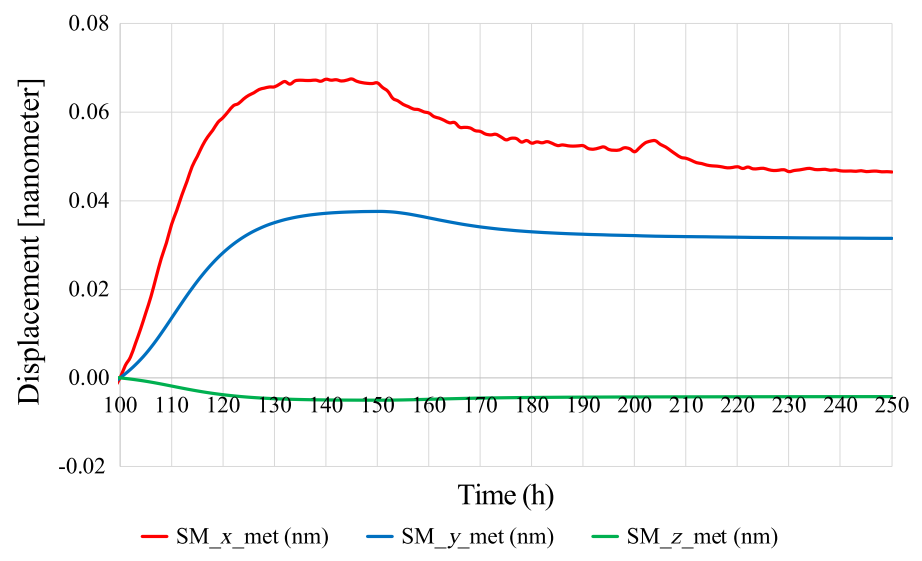

Fig. 48 Displacements of SM due to thermal DRM.

Table 11 Predicted maximum uncorrectable "drift" motion of PM and SM for DRM.

\begin{tabular}{lcccccc}
\hline \hline DOF & $\Delta x(\mathrm{~nm})$ & $\Delta y(\mathrm{~nm})$ & $\Delta z(\mathrm{~nm})$ & $\Theta x(\mathrm{nrad})$ & $\Theta y(\mathrm{nrad})$ & $\Theta z(\mathrm{nrad})$ \\
\hline Primary & 0.71 & 0.48 & 0.05 & 0.25 & 0.38 & 0.39 \\
Secondary & 0.07 & 0.04 & 0.01 & 0.01 & 0.04 & 0.29 \\
\hline \hline
\end{tabular}

Table 11 lists the maximum amplitude of the uncorrectable motion for each rigid-body DOF of the PM and SM. These motions predict an on-sky LOS jitter of $<0.2$ mas (i.e., $\sim 1.5 \times$ margin). In this case, no MUF is applied.

\subsubsection{Predicted LOS stability performance}

rss'ing the rigid-body motions of the PM and SM from the mechanical and thermal model STOP analysis (Tables 11 and 12) predicts their maximum rigid-body motions (Table 12). These motions predict an on-sky LOS stability of $<0.2$ mas for a $1.5 \times$ margin.

\subsection{Predicted WFE Stability}

WFE stability specification is driven by the coronagraph. Any temporal or dynamic change in WFE can result in dark hole speckles that produce a false exoplanet measurement or mask a true signal. Figure 10 presents a simple VVC-6 error budget that allocates WFE stability between LOS, inertial, and thermal instability.

One potential mitigation for LOS vibration and inertial instability is that mechanical motions tend to be periodic, and multiple cycles over an integration period will produce a fixed pattern. If this pattern is $100 \%$ repeatable, it is possible to remove it through speckle subtraction. But, if the motion is not periodic, there will be a nonrepeatable component that cannot be calibrated and removed. Thus, it is the instability of the mechanical motion (not the motion itself) that is important. Regardless, the best mitigation strategy is to minimize WFE instability.

Table 12 Predicted maximum RBM of PM and SM for a DRM.

\begin{tabular}{lcccccc}
\hline \hline DOF & $\Delta x(\mathrm{~nm})$ & $\Delta y(\mathrm{~nm})$ & $\Delta z(\mathrm{~nm})$ & $\Theta x(\mathrm{nrad})$ & $\Theta y(\mathrm{nrad})$ & $\Theta z(\mathrm{nrad})$ \\
\hline Primary & 0.74 & 0.49 & 0.17 & 0.25 & 0.38 & 0.39 \\
Secondary & 0.67 & 0.58 & 0.03 & 0.05 & 0.07 & 0.33 \\
\hline \hline
\end{tabular}




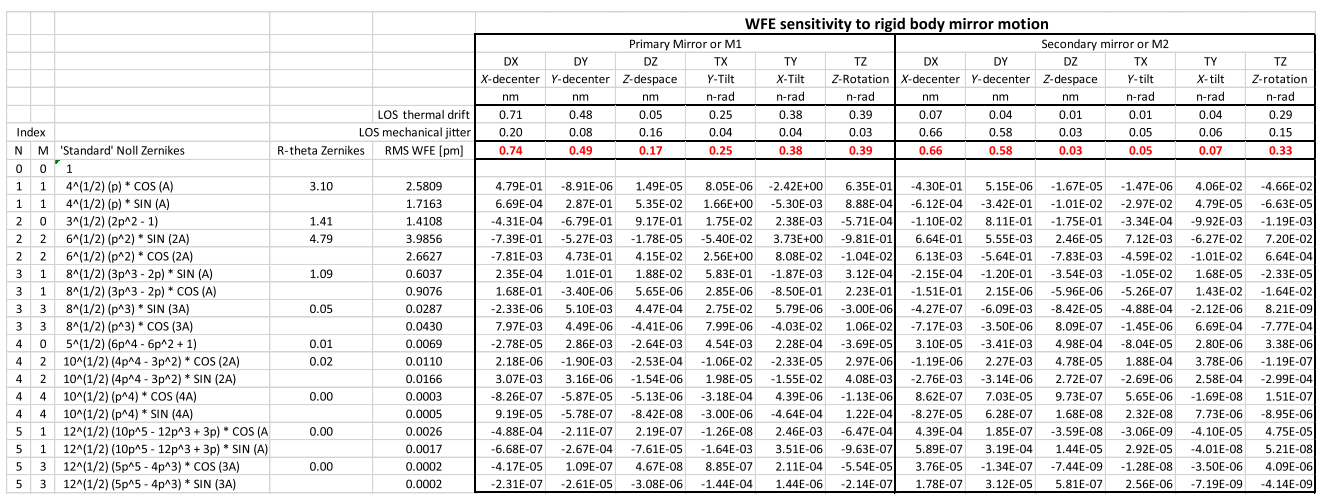

Fig. 49 WFE decomposed into Zernike polynomial coefficients for PM and SM rigid-body motions.

\subsubsection{Predicted LOS WFE stability}

LOS WFE instability is produced by LOS drift and jitter. Thermal LOS drift and mechanical LOS jitter causes beam shear on the SM and TM. Also, because these mirrors are conics, beam shear manifests itself as low-order astigmatism and coma. Conic mirrors correct spherical aberration, and the shear of full aperture spherical aberration is coma. When viewed over an off-axis subaperture, coma appears as astigmatism. As shown in Fig. 49, each mirror's RBM produces its own WFE "signature" that can decompose into a set of Zernike polynomial coefficients. As expected, there are no significant higher-order errors. The input for the analysis in Fig. 49 is the rss'd rigid body displacements in Table 12.

Figure 50 compares the predicted LOS WFE calculated in Fig. 49 to the LOS WFE allocation in the simple VVC-6 error budget of Fig. 10. As expected, the largest LOS errors are tilt, power, and astigmatism. However, these errors are not significant to the VVC-6. The most significant are trefoil and secondary astigmatism. But, because the LOS jitter is so small, the predicted amplitudes for these errors have $>100 \times$ margin relative to their error budget allocation.

\subsubsection{Predicted inertial WFE stability}

Inertial WFE instability occurs when the PM is accelerated by mechanical disturbances causing it to react (i.e., bend) against its mounts. Figures 24 and 25 show two such mechanical

\begin{tabular}{|c|c|c|c|c|c|}
\hline & & & & & LOS \\
\hline \multicolumn{2}{|c|}{ Index } & & \multirow{2}{*}{$\begin{array}{c}\text { Allocation LOS } \\
\text { [pm rms] }\end{array}$} & & RSS WFE \\
\hline \multirow[t]{2}{*}{$\mathrm{N}$} & $M$ & Aberration & & Margin & (pm rms) \\
\hline & & Total RMS & 2528 & 1430 & 1.767 \\
\hline 1 & 1 & Tilt & 1351.83 & 1984 & 0.681 \\
\hline 2 & 0 & Power (defocus) & 1010.98 & 837 & 1.208 \\
\hline 2 & 2 & Astigmatism & 1224.08 & 1145 & 1.069 \\
\hline 3 & 1 & Coma & 1089.60 & 4547 & 0.240 \\
\hline 4 & 0 & Spherical & 925.42 & 212904 & 0.004 \\
\hline 3 & 3 & Trefoil & 1.63 & 141 & 0.012 \\
\hline 4 & 2 & Sec astigmatism & 0.88 & 201 & 0.004 \\
\hline 5 & 1 & Sec coma & 0.80 & 1179 & 0.001 \\
\hline 6 & 0 & Sec spherical & 0.60 & 42835 & 0.000 \\
\hline 4 & 4 & Tetrafoil & 1.57 & 11780 & 0.000 \\
\hline 5 & 3 & Sec trefoil & 0.73 & 12189 & 0.000 \\
\hline 6 & 2 & Ter astigmatism & 0.45 & 29360 & 0.000 \\
\hline 7 & 1 & Ter coma & 0.38 & 229124 & 0.000 \\
\hline 5 & 5 & Pri pentafoil & 0.55 & 356736 & 0.000 \\
\hline 6 & 4 & Sec tetrafoil & 0.56 & 740369 & 0.000 \\
\hline 7 & 3 & Ter trefoil & 0.41 & 1376489 & 0.000 \\
\hline 6 & 6 & Hexafoil & 0.39 & 3944935 & 0.000 \\
\hline 7 & 5 & Sec pentafoil & 0.38 & 4982996 & 0.000 \\
\hline 7 & 7 & Pri septafoil & 0.44 & 6511622 & 0.000 \\
\hline
\end{tabular}

Fig. 50 LOS WFE stability predicted to have $>100 \times$ margin relative to error budget allocation. 


\section{HabEx rev2 primary mirror ( $1^{\text {st }}$ normal mode) frequency $=43.564 \mathrm{~Hz}$}

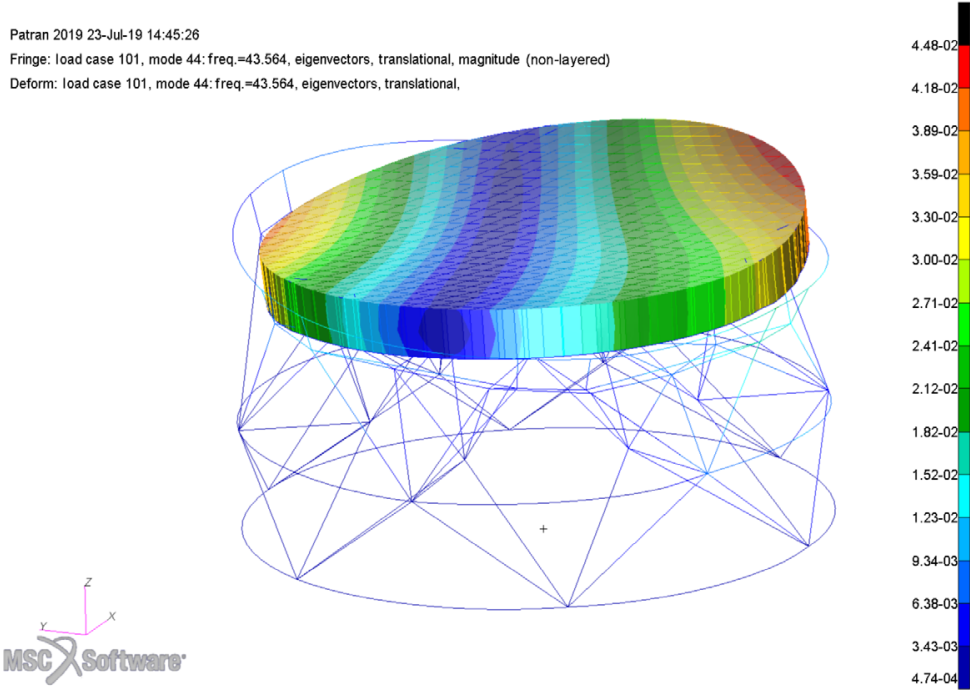

Fig. 51 Mirror bending from 49.5- $\mathrm{Hz}$ rocking mode.

disturbances-43.5-Hz rocking and $50-\mathrm{Hz}$ bouncing. Figures 51 and 52 show how the mirror bends as it reacts against the hexapod mount for the rocking and bouncing modes. Note that inertial WFE is not the same as modal resonant motion. Two methods were used to predict inertial WFE instability: linear scaling of gravity sag deformation and predicted deformation using NASTRAN decomposed into Zernike polynomials using SigFit.

Linear scaling of gravity sag deformation. To first order, inertial WFE should be similar to the mirror's static $(X, Y, Z)$ gravity sag (i.e., bending of the mirror when it is exposed to a $1-\mathrm{G}$ acceleration in the $X, Y, Z$ directions) (Fig. 23), scaled by the on-orbit acceleration to which it is exposed. To estimate on-orbit acceleration, the observatory FEM was used to calculate $X, Y, Z$-accelerate from the microthrusters at each PM hexapod interface. Figure 53 shows the maximum calculated $X, Y, Z$-acceleration into the mirror at each frequency. Nevertheless,

\section{HabEx rev2 primary mirror ( $3^{\text {rd }}$ normal mode) \\ frequency $=50.605 \mathrm{~Hz}$}

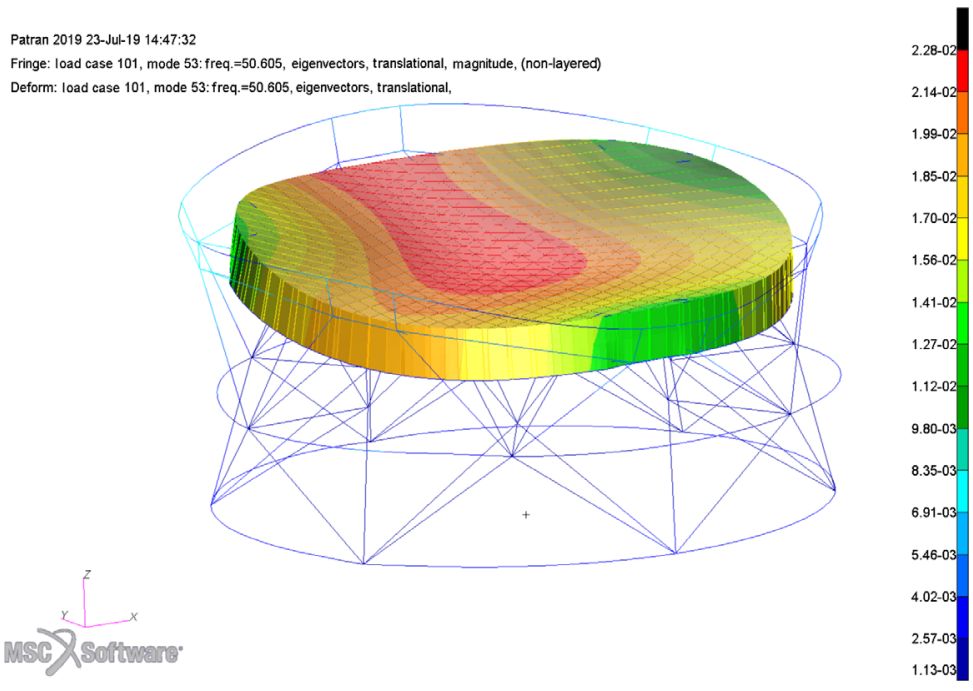

Fig. 52 Mirror bending from $50-\mathrm{Hz}$ bouncing mode. 


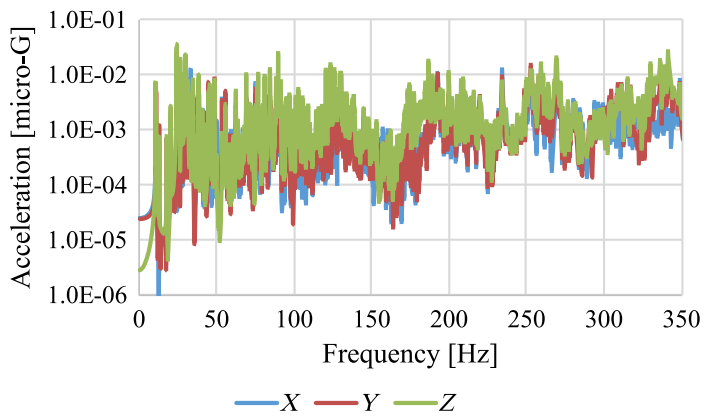

Fig. 53 Maximum $\mathrm{PM}(X, Y, Z)$ acceleration at each frequency.

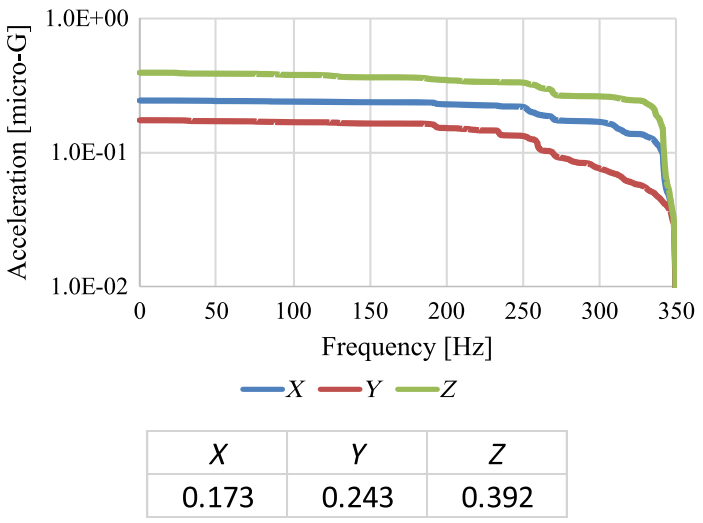

Fig. 54 Maximum $\mathrm{PM}(X, Y, Z)$ acceleration at each frequency.

because microthrusters are always on, it is necessary to rss over all frequencies to determine the maximum acceleration into the mirror (Fig. 54). The absolute maximum $X, Y, Z$ acceleration imparted into the mirror is $(170,240$, and $390 \mathrm{nG})$. Scaling the RMS gravity sag WFE of (37.3, 36.8, and $25.2 \mu \mathrm{m})$ yields an $X, Y, Z$ RMS inertial WFE of $(6.3,8.8$, and $9.8 \mathrm{pm})$. Assuming that $X$ and $Y$ gravity sags are entirely astigmatism and the $Z$-sag is trefoil, this first-order linear analysis indicates that the PM will have $\sim 11 \mathrm{pm}$ of astigmatism and $\sim 10 \mathrm{pm}$ of trefoil. Obviously, the trefoil is about $10 \times$ higher than allowed by the simple error budget.

Fortunately, this analysis is conservative. The maximum acceleration amplitudes at each frequency are $\sim 10 \times$ larger than the RMS acceleration at each frequency. In addition, the analysis does not assume mass dampening. The analysis assumes that the PM responds to all acceleration frequencies equally. In practice, this is not real. In a real system, the PM's inertia prevents responding to high frequency acceleration.

Predicted inertial surface deformation. To obtain a more accurate estimate of inertial WFE, NASTRAN calculated the displacement of PM surface nodes from 0 to $350 \mathrm{~Hz}$ as a function of the microthruster noise specification applied to the structure from 0 to $10 \mathrm{HZ}$ (multiplied times 2 for wavefront). To eliminate rigid body displacements, the node displacements were calculated relative to a fixed surface reference point. The WFE produced by these displacements were fit to Zernike polynomials using SigFig. Because the microthrusters excite all modes simultaneously, the individual frequencies were rss'd to yield a total accumulated WFE for each Zernike term (Fig. 55). Consistent with mass dampening (i.e., where the mirror has a smaller response to higher frequency accelerations), the nonlinear analysis predicts an astigmatism WFE that is $3 \times$ smaller, a focus error that is $4 \times$ smaller, and a trefoil WFE that is $5 \times$ smaller than the simple linear gravity sag scaling approach. Figure 56 shows how the calculated inertial WFE of the PM compares with its simple error budget allocation of Fig. 10. As expected, trefoil is the dominant term, but still has $1.6 \times$ margin. 


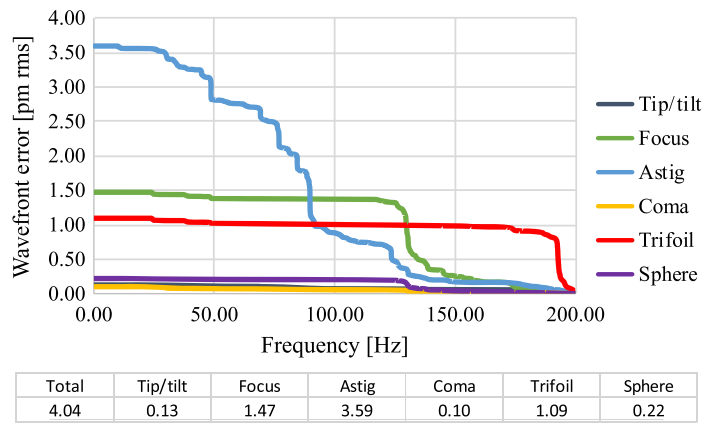

Fig. 55 Total PM inertial deformation of select Zernikes: astig $=3.6 \mathrm{pm} \mathrm{rms;} \mathrm{focus}=1.5 \mathrm{pm} \mathrm{rms;}$ trefoil $=1.1 \mathrm{pm}$ rms.

\begin{tabular}{|c|c|c|c|c|c|}
\hline & & & \multicolumn{3}{|c|}{ Inertial WFE stability } \\
\hline & & & Allocation & & Zernikes \\
\hline \multicolumn{2}{|c|}{ Index } & & Inertial & Margin & [pm rms] \\
\hline \multirow[t]{2}{*}{$\mathrm{N}$} & M & Aberration & {$[\mathrm{pm} \mathrm{rms}]$} & & \\
\hline & & Total RMS & 891.94 & & 3.994 \\
\hline 1 & 1 & Tilt & 1351.83 & 10990.5 & 0.123 \\
\hline 2 & 0 & Power (defocus) & 1010.98 & 707.0 & 1.430 \\
\hline 2 & 2 & Astigmatism & 1224.08 & 343.9 & 3.559 \\
\hline 3 & 1 & Coma & 1089.60 & 11006.1 & 0.099 \\
\hline 4 & 0 & Spherical & 925.42 & 4344.7 & 0.213 \\
\hline 3 & 3 & Trefoil & 1.63 & 1.6 & 1.039 \\
\hline 4 & 2 & Sec astigmatism & 0.88 & 5.0 & 0.178 \\
\hline 5 & 1 & Sec coma & 0.80 & 30.8 & 0.026 \\
\hline 6 & 0 & Sec spherical & 0.60 & 21.5 & 0.028 \\
\hline 4 & 4 & Tetrafoil & 1.57 & 7.9 & 0.198 \\
\hline 5 & 3 & Sec trefoil & 0.73 & 6.5 & 0.112 \\
\hline 6 & 2 & Ter astigmatism & 0.45 & 21.6 & 0.021 \\
\hline 7 & 1 & Ter coma & 0.38 & 11.4 & 0.033 \\
\hline 5 & 5 & Pentafoil & 0.55 & 7.4 & 0.074 \\
\hline 6 & 4 & Sec tetrafoil & 0.56 & 19.3 & 0.029 \\
\hline 7 & 3 & Ter trefoil & 0.41 & 27.5 & 0.015 \\
\hline 6 & 6 & Hexafoil & 0.39 & 15.0 & 0.026 \\
\hline 7 & 5 & Sec pentafoil & 0.38 & 25.1 & 0.015 \\
\hline 7 & 7 & Septafoil & 0.44 & 43.5 & 0.010 \\
\hline
\end{tabular}

Fig. 56 Estimated inertial WFE.

\subsubsection{Predicted thermal WFE stability}

Thermal WFE instability occurs when the PM's bulk temperature or temperature gradient changes. If the mirror's CTE is completely homogeneous and constant, then a bulk temperature should only result in a defocus error. But any inhomogeneity in the mirror's CTE will result in a temperature-dependent WFE. In addition, because CTE is itself temperature dependent, any change in the mirror's thermal gradient will also result in a WFE. Unlike mechanical WFE, which is mostly low-spatial-frequency, thermal WFE can have significant high-spatial-frequency content. Therefore, the best mitigation strategy is to minimize thermal disturbances. This can be done either by passive isolation or active sense and control.

Thermal WFE instability was predicted by applying the DRM discussed in Sec. 4.1.4 to the integrated model. Thermal Desktop calculated the temperature distribution as a function of time. With this distribution, NASTRAN calculated surface deformations, which were then used by SigFit to decompose the temporal WFE into Zernike polynomials as a function of time.

J. Astron. Telesc. Instrum. Syst. $\quad$ 034004-40 Jul-Sep 2020 • Vol. 6(3) 


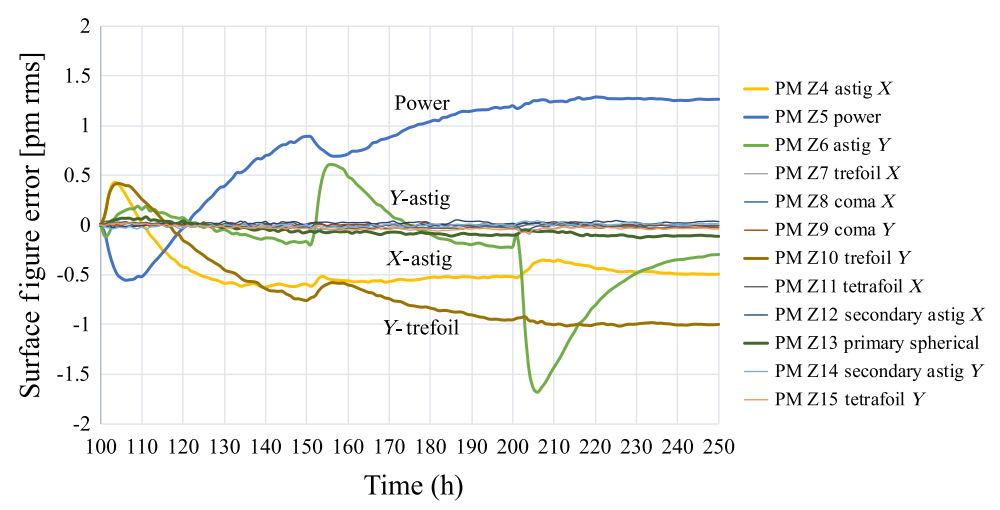

Fig. 57 DRM PM SFE changes.

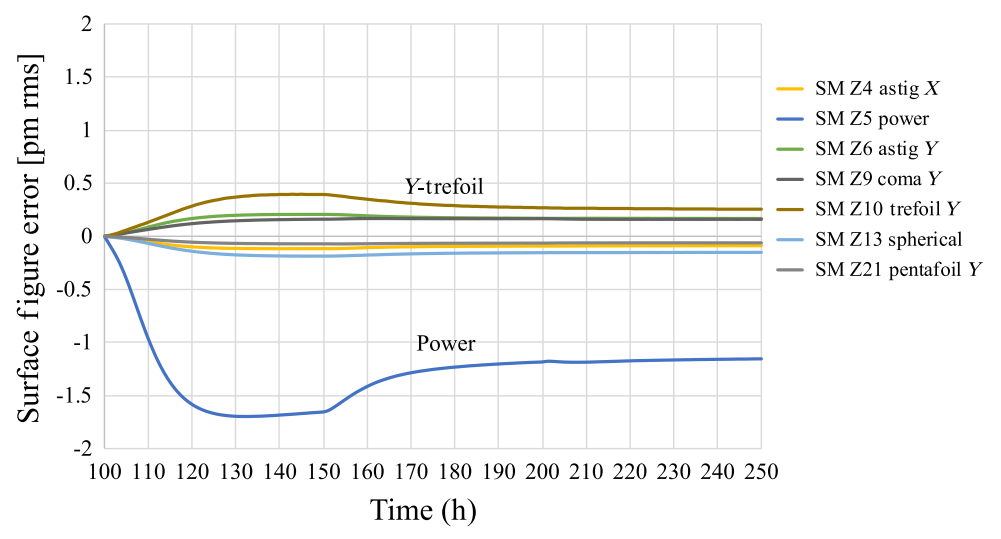

Fig. 58 DRM SM SFE changes.

Figure 57 shows the predicted change in PM SFE decomposed into Zernike polynomials. As symmetric errors, power and trefoil's changes are caused by the DRM pitch angle, which changes the total solar load on the telescope. Moreover, as an asymmetric error, astigmatism's change is caused by the DRM roll that shifts thermal load from one side to the other. Figure 58 shows predicted change for the SM. Because of its location, it is relatively insensitive to roll.

Total DRM WFE was calculated by rss'ing the PM and SM Zernike terms as a function of time and selecting the maximum amplitude for each. This analysis has a $3 \times \mathrm{X}$ MUF. Figure 59 shows each Zernike compared to the simple error budget of Fig. 10. Obviously, trefoil is a problem. Fortunately, there is a solution: reallocate the error budget.

The thermal STOP analysis pipeline does not evaluate as many of the higher-order Zernike terms as the optomechanical STOP analysis pipeline. Therefore, there are some "holes" in Fig. 59 data analysis. However, it is reasonable to assume that these terms will be similar in amplitude to those reported and the $3 \times$ MUF is an analysis artifact. The thermal STOP model used PV CTE homogeneity measured WFE instead of RMS. But, because there is an interaction between bulk CTE and CTE homogeneity, the results were not "scaled."

\section{Performance Error Budget}

The initial error budget defined in Sec. 2.3 and shown in Fig. 10 assumed a simple uniform distribution of contrast leakage. However, because some Zernike terms are more likely to occur than other terms, it is permissible to reallocate contrast leakage from the less-likely terms to the more-likely terms. Figure 60 shows an optimized error budget for the VVC-6 where the majority of the contrast leakage is allocated to trefoil. The first set of columns lists the Zernike polynomial 


\begin{tabular}{|c|c|c|c|c|c|}
\hline & & & \multicolumn{3}{|c|}{ Thermal WFE stability } \\
\hline & & & Allocation & & Zernike \\
\hline \multicolumn{2}{|c|}{ Index } & & Thermal & Margin & {$[\mathrm{pm} \mathrm{rms}]$} \\
\hline \multirow[t]{2}{*}{$\mathrm{N}$} & M & Aberration & [pm rms] & & \\
\hline & & Total RMS & 2528.15 & & 5.565 \\
\hline 1 & 1 & Tilt & 1351.83 & 51993.3 & 0.026 \\
\hline 2 & 0 & Power (defocus) & 1010.98 & 268.9 & 3.759 \\
\hline 2 & 2 & Astigmatism & 1224.08 & 353.5 & 3.463 \\
\hline 3 & 1 & Coma & 1089.60 & 3158.3 & 0.345 \\
\hline 4 & 0 & Spherical & 925.42 & 2285.0 & 0.405 \\
\hline 3 & 3 & Trefoil & 1.63 & 0.8 & 2.098 \\
\hline 4 & 2 & Sec astigmatism & 0.88 & 8.2 & 0.108 \\
\hline 5 & 1 & Sec coma & 0.80 & 7.6 & 0.105 \\
\hline 6 & 0 & Sec spherical & 0.60 & & \\
\hline 4 & 4 & Tetrafoil & 1.57 & 8.3 & 0.189 \\
\hline 5 & 3 & Sec trefoil & 0.73 & 3.1 & 0.233 \\
\hline 6 & 2 & Ter astigmatism & 0.45 & & \\
\hline 7 & 1 & Ter coma & 0.38 & & \\
\hline 5 & 5 & Pentafoil & 0.55 & 2.5 & 0.217 \\
\hline 6 & 4 & Sec tetrafoil & 0.56 & & \\
\hline 7 & 3 & Ter trefoil & 0.41 & & \\
\hline 6 & 6 & Hexafoil & 0.39 & & \\
\hline 7 & 5 & Sec pentafoil & 0.38 & & \\
\hline 7 & 7 & Septafoil & 0.44 & & \\
\hline
\end{tabular}

Fig. 59 Estimated PM/SM thermal WFE.

\begin{tabular}{|c|c|c|c|c|c|c|c|c|c|c|c|}
\hline \multicolumn{2}{|c|}{ Index } & & \multicolumn{3}{|c|}{ Predicted performance amplitude [pm rms] } & \multirow{2}{*}{$\begin{array}{c}\text { Total WFE } \\
\text { [pm rms] }\end{array}$} & \multirow{2}{*}{$\begin{array}{c}\text { VVC-6 sensitivity } \\
{[\mathrm{ppt} / \mathrm{pm} \mathrm{PV}]}\end{array}$} & \multirow{2}{*}{$\begin{array}{c}\text { Raw contrast } \\
\text { [ppt] }\end{array}$} & \multirow{2}{*}{$\begin{array}{c}\text { Allocation } \\
\text { [ppt] }\end{array}$} & \multirow{2}{*}{\begin{tabular}{|c|} 
WFE tolerance \\
[pm RMS]
\end{tabular}} & \multirow[t]{2}{*}{ Margin } \\
\hline $\mathrm{N}$ & M & Aberration & LOS & Inertial & Thermal & & & & & & \\
\hline & & Total RMS & 5.715 & 3.994 & 5.565 & 8.921 & & 7.289 & 30.000 & 36.715 & \\
\hline 1 & 1 & Tilt & 3.025 & 0.123 & 0.026 & 3.027 & 0.0002 & 0.001 & 0.005 & 12.459 & 4.1 \\
\hline 2 & 0 & Power (defocus) & 0.728 & 1.430 & 3.759 & 4.087 & 0.0003 & 0.002 & 0.010 & 16.821 & 4.1 \\
\hline 2 & 2 & Astigmatism & 4.674 & 3.559 & 3.463 & 6.819 & 0.0002 & 0.003 & 0.013 & 28.066 & 4.1 \\
\hline 3 & 1 & Coma & 1.064 & 0.099 & 0.345 & 1.123 & 0.0002 & 0.001 & 0.002 & 4.620 & 4.1 \\
\hline 4 & 0 & Spherical & 0.005 & 0.213 & 0.405 & 0.458 & 0.0003 & 0.000 & 0.001 & 1.883 & 4.1 \\
\hline 3 & 3 & Trefoil & 0.050 & 1.039 & 2.098 & 2.342 & 1.0016 & 6.634 & 27.303 & 9.638 & 4.1 \\
\hline 4 & 2 & Sec astigmatism & 0.019 & 0.178 & 0.108 & 0.209 & 1.6495 & 1.091 & 4.489 & 0.861 & 4.1 \\
\hline 5 & 1 & Sec coma & 0.003 & 0.026 & 0.105 & 0.108 & 1.6645 & 0.624 & 2.568 & 0.445 & 4.1 \\
\hline 6 & 0 & Sec spherical & 0.000 & 0.028 & 0.000 & 0.028 & 2.8902 & 0.214 & 0.881 & 0.115 & 4.1 \\
\hline 4 & 4 & Tetrafoil & 0.001 & 0.198 & 0.189 & 0.274 & 0.9312 & 0.806 & 3.317 & 1.127 & 4.1 \\
\hline 5 & 3 & Sec trefoil & 0.000 & 0.112 & 0.233 & 0.259 & 1.8200 & 1.630 & 6.708 & 1.064 & 4.1 \\
\hline 6 & 2 & Ter astigmatism & 0.000 & 0.021 & 0.000 & 0.021 & 2.7219 & 0.214 & 0.880 & 0.086 & 4.1 \\
\hline 7 & 1 & Ter coma & 0.000 & 0.033 & 0.000 & 0.033 & 3.0608 & 0.404 & 1.663 & 0.136 & 4.1 \\
\hline 5 & 5 & Pentafoil & 0.000 & 0.074 & 0.217 & 0.229 & 2.4409 & 1.939 & 7.979 & 0.944 & 4.1 \\
\hline 6 & 4 & Sec tetrafoil & 0.000 & 0.029 & 0.000 & 0.029 & 2.2050 & 0.239 & 0.985 & 0.119 & 4.1 \\
\hline 7 & 3 & Ter trefoil & 0.000 & 0.015 & 0.000 & 0.015 & 2.7946 & 0.168 & 0.690 & 0.062 & 4.1 \\
\hline 6 & 6 & Hexafoil & 0.000 & 0.026 & 0.000 & 0.026 & 3.1667 & 0.308 & 1.268 & 0.107 & 4.1 \\
\hline 7 & 5 & Sec pentafoil & 0.000 & 0.015 & 0.000 & 0.015 & 3.0694 & 0.184 & 0.758 & 0.062 & 4.1 \\
\hline 7 & 7 & Septafoil & 0.000 & 0.010 & 0.000 & 0.010 & 2.6510 & 0.106 & 0.436 & 0.041 & 4.1 \\
\hline
\end{tabular}

Fig. 60 Optimized error budget for VVC-6.

indices and common names. The first three data columns input the predicted STOP performance for each error source with its MUF (4× MUF on LOS Jitter and LOS wavefront stability, $2 \times$ MUF on inertial WFE stability, and $3 \times$ MUF on thermal WFE stability). These are rss'd to yield a total RMS WFE for each Zernike term. The VVC-6 sensitivity column shows how much ppt of raw contrast error leaks through the VVC-6 coronagraph for a picometer of Zernike WFE. The raw contrast column shows the predicted leakage for the STOP predicted Zernike WFE. The allocation column normalizes and redistributes the $30 \mathrm{ppt}$ of allowed raw contrast to maximize the amount allocated to trefoil. The WFE tolerance column is the new error budget. This error budget provides a margin of $4.1 \times$ for all Zernike. Note that this error budget is ONLY for the baseline ZERODUR ${ }^{\circledR}$ PM. A different mirror substrate or mount design will require a different error budget. And the error budget will need to be adjusted for the "as-built" mirror. 


\section{Predicted Impulse Response}

Ring-down time or impulse response is how long it takes for the telescope to stabilize its LOS and WFE after a slew maneuver. Transient dynamic analysis was performed to predict slew ring down time via finite element analysis using MSC Patran as the pre/postprocessor and MSC NASTRAN as the solver. ${ }^{71}$ To simulate a nominal pitch maneuver, an $8.8-\mathrm{N}$ thrust was applied as a 20.5-s step function at the $Y$ axis thrusters. After $368 \mathrm{~s}$, another was applied in the opposite direction to stop the slew (Fig. 61).

The relative motion between the PM and SM was calculated for $300 \mathrm{~s}$ beyond the termination of the second thrust (Fig. 62). Note that no MUF was applied to this analysis.
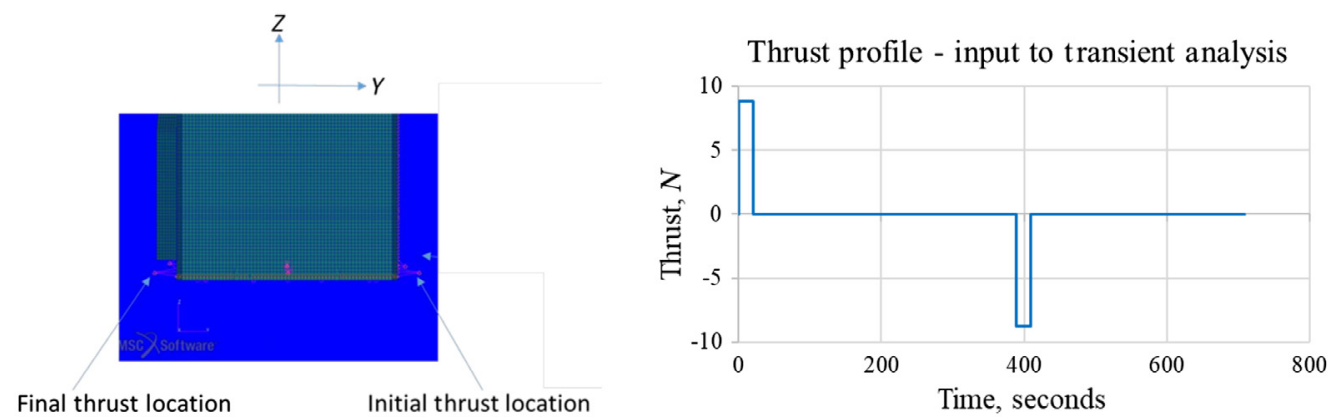

Fig. 61 Slew impulses applied to $Y$-axis by thruster.
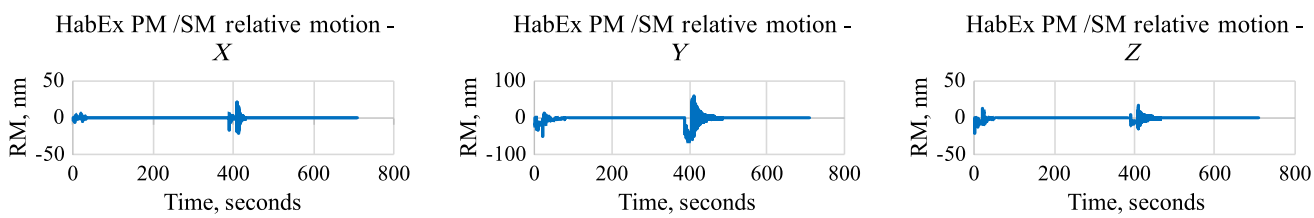

HabEx PM /SM relative motion-
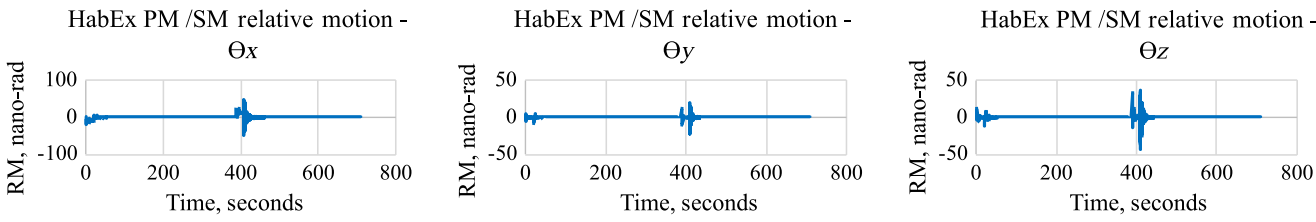

Fig. 62 Relative motion between PM and SM caused by thruster impulses.
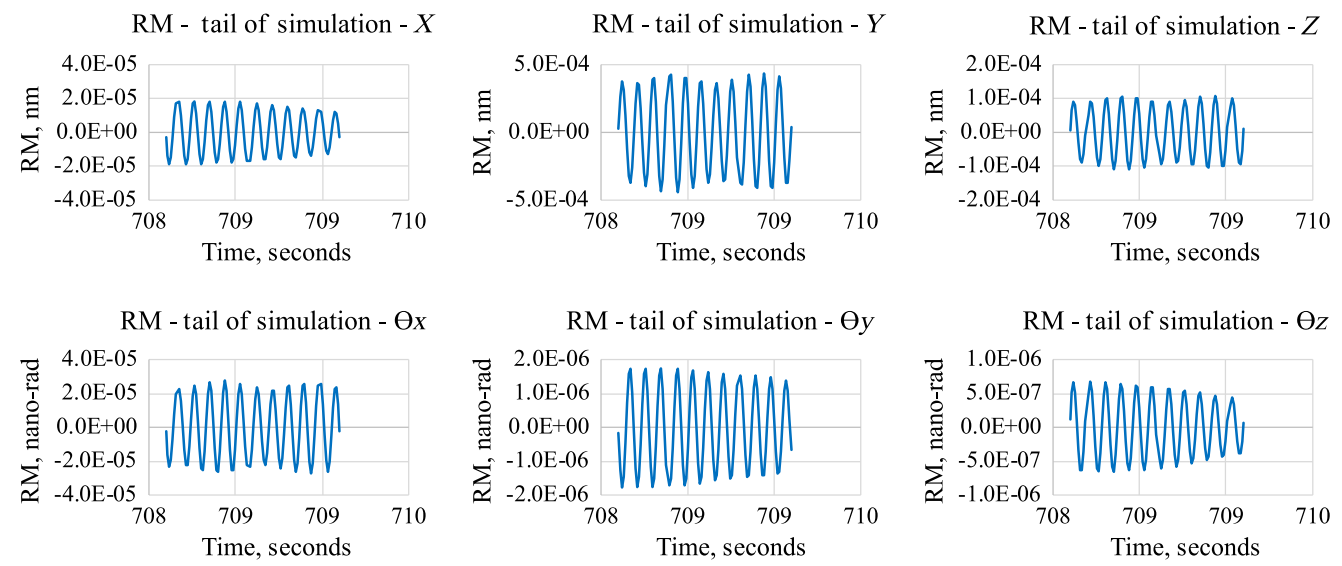

Fig. 63 After $300 \mathrm{~s}$, relative motion between PM and SM caused by thruster impulses is $<1 \mathrm{pm}$. 
Because the baseline telescope structure is very stiff, after $300 \mathrm{~s}$ of ring-down, the largest predicted relative motion between the PM and SM is $<1 \mathrm{pm}$ (Fig. 63). Thus, telescope slews will have negligible impact on the settle time before performing science observations.

\section{Conclusion}

HabEx is under study for the 2020 Astrophysics Decadal Survey. Its goal is to image and spectroscopically characterize planetary systems around nearby Sun-like stars. A large, ultrastable UVOIR telescope is critical to achieving the HabEx science goals. The desired telescope is a 4-m off-axis, unobscured TMA configuration with diffraction-limited performance at $400 \mathrm{~nm}$ and wavefront stability on the order of a few tens of pm. The baseline HabEx telescope is designed using standard engineering practices and its design closes. The telescope's predicted STOP meets with its specified performance error budget allocations for LOS jitter, LOS WFE, inertial WFE, and thermal WFE (Fig. 61). The choice to use microthrusters for pointing control instead of reaction wheels is key to meeting its LOS and inertial specifications. The baseline observatory design fits with margin within the mass and volume constraints of the SLS Block-2 8.4-m fairing.

\section{Acknowledgments}

This paper was the work of the NASA MSFC HabEx Team and our JPL Collaborators. MSFC Team: Thomas Brooks, Jacqueline Davis, Michael Effinger, Brent Knight, Scott Smith, Mark Stahl; Willian Arnold (AI Solution); Mike Baysinger (ESSCA), Jay Garcia (ESSCA), Mary Caldwell Ron Hunt, Andrew Singleton, and Melissa Therrell (ESSA); Bijan Nemati (University of Alabama in Huntsville); and interns Jonathan Gaskin (University of North Carolina in Charlotte), Jonathan McCready (North Carolina State University), and Hao Tang (University of Michigan). JPL Team: Keith Warfield, Gary Kuan, Velibor Cormarkovic, Scott Howe, Stefan Martin, Navtej Saini, Stuart Shaklan, Juan Villalvazo, and Team X. The authors would also like to thank Nicholas Reich, technical editor through ASRC Federal's communications contract with NASA MSFC.

\section{References}

1. The National Academies of Sciences, Engineering, and Medicine, New Worlds, New Horizons in Astronomy and Astrophysics, The National Academies Press, Washington, D.C. (2010).

2. NASA, NASA Space Technology Roadmaps and Priorities: Restoring NASA's Technological Edge and Paving the Way for a New Era in Space, The National Academies Press, Washington, D.C. (2012).

3. NASA, Habitable Exoplanet Observatory: Exploring New Worlds, Understanding Our Universe, NASA, Washington, D.C. (2019). https://www.jpl.nasa.gov/habex/pdf/HabExFinal-Report-Public-Release.pdf.

4. J. E. Harvey and F. Christ, "Diffraction effects of telescope secondary mirror spiders on various image-quality criteria," Appl. Opt. 34(28), 6337-6349 (1995).

5. B. Nemati et al., "HabEx Telescope WFE stability specification derived from coronagraph starlight leakage," Proc. SPIE 10743, 107430 G (2018).

6. B. Nemati et al., "Method for deriving optical telescope performance specifications for Earth-detecting coronagraphs," J. Astron. Telesc. Instrum. Syst. (2020).

7. R. H. Morgan et al., "HabEx yield modeling with for systems engineering (Conference Presentation)," Proc. SPIE 10398, 1039804 (2017).

8. NASA: Exoplanet Program, Exo-C: Imaging Nearby Worlds, NASA, Washington, D.C. (2015). https://exep.jpl.nasa.gov/stdt/Exo-C_Final_Report_for_Unlimited_Release_150323 .pdf.

9. J. Krist, Researcher at the Jet Propulsion Laboratory, private communication (2019). 
10. P. H. Stahl et al., "Survey of interferometric techniques used to test JWST optical components," Proc. SPIE 7790, 779002 (2010).

11. S. B. Shaklan, J. J. Green, and D. M. Palacios, "The Terrestrial Planet Finder Coronagraph optical surface requirements," Proc. SPIE 6265, 62651I (2006).

12. S. B. Shaklan and J. J. Green, "Reflectivity and optical surface height requirements in a broadband coronagraph.1.Contrast floor due to controllable spatial frequencies," Appl. Opt. 45(21), 5143-5153 (2006).

13. T. E. Brooks et al., "Modeling the Extremely Lightweight Zerodur Mirror (ELZM) thermal soak test," Proc. SPIE 10374, 103740E (2017).

14. S. Shaklan, Researcher at the Jet Propulsion Laboratory, private communication (2017).

15. H. P. Stahl, "Habitable exoplanet imager optical telescope concept design," Proc. SPIE 10398, 1039806 (2017).

16. G. J. Ruane, NASA Jet Propulsion Laboratory with the California Institute of Technology, private communication (2017).

17. H. P. Stahl, "Overview and performance prediction of the baseline 4-meter telescope concept design for the habitable-zone exoplanet observatory," Proc. SPIE 10698, 106980W (2018).

18. H. P. Stahl et al., "Designing astrophysics missions for NASA's Space Launch System," J. Astronom. Telesc. Instrum. Syst. 2(4), 041213 (2016).

19. H. P. Stahl et al., "Habitable-zone exoplanet observatory (HabEx) baseline 4-m telescope design and predicted performance," Proc. SPIE 11115, $111150 \mathrm{U}$ (2019).

20. M. S. Lake, L. D. Peterson, and M. B. Levine, "Rationale for defining structural requirements for Large Space Telescopes," J. Spacecr. Rockets 39(5) (2002).

21. G. Mosier, "Isolation requirement," NASA, AMTD Report (an internal document) (2014).

22. L. D. Feinberg et al., "A cost-effective and serviceable ATLAST 9.2m telescope architecture," Proc. SPIE 9143, 914316 (2014).

23. J. B. Knight et al., "Dynamic/jitter assessment of multiple potential HabEx structural designs," Proc. SPIE 10374, 1037402 (2017).

24. H. P. Stahl, "Advanced Mirror Technology Development (AMTD): year five status," Proc. SPIE 10401, 1040100 (2017).

25. H. P. Stahl, "Advanced Mirror Technology Development (AMTD) project: overview and year four accomplishments," Proc. SPIE 9912, 99120S (2016).

26. H. P. Stahl, "Overview and accomplishments of Advanced Mirror Technology Development Phase 2 (AMTD-2) project," Proc. SPIE 9602, 960208 (2015).

27. H. P. Stahl et al., "Advanced Mirror Technology Development (AMTD) project: 2.5 year status," Proc. SPIE 9143, 91431S (2014).

28. H. P. Stahl et al., "Overview and recent accomplishments of the Advanced Mirror Technology Development (AMTD) for large aperture UVOIR space telescopes project,' Proc. SPIE 8860, 88600Q (2013).

29. J. E. Krist et al., "End-to-end coronagraphic modeling including a low-order wavefront sensor," Proc. SPIE 8422, 844253 (2012).

30. L. Leboulleux et al., "Pair-based analytical model for segmented telescopes imaging from space for sensitivity analysis," J. Astron. Telesc. Instrum. Syst. 4(3), 035002 (2018).

31. B. Nemati et al., "Effects of space telescope primary mirror segment errors on coronagraph instrument performance," Proc. SPIE 10398, 103980G (2017).

32. M. T. Stahl, S. B. Shaklan, and H. P. Stahl, "Preliminary analysis of effect of random segment errors on coronagraph performance," Proc. SPIE 9605, 96050P (2015).

33. National Research Council, A Scientific Assessment of a New Technology Orbital Telescope, The National Academies Press, Washington, D.C. (1995).

34. B. Honneger, "NPS new home for Giant Segmented-Mirror Space Telescope," Naval Postgraduate School, 2010, https://web.nps.edu/About/News/NPS-New-Home-for-GiantSegmented-Mirror-Space-Telescope-html.

35. T. Westerhoff and T. Hull, "Production of $4 \mathrm{~m}$ diameter Zerodur ${ }^{\circledR}$ mirror substrates," HabEx White Paper Contribution, Schott AG, Mainz, Germany (2018).

36. C. Cox, "Collins Aerospace inputs and assessment for the HabEx primary mirror assembly," Document PA-2227 (2018). 
37. D. Sheikh, Mirror Coating Technology for HabEx, ZeCoat Corporation (2018).

38. T. Hull, Professor of physics and astronomy at the University of New Mexico, private communication (2018).

39. M. Edwards, Researcher at Corning Inc., private communication (2018).

40. B. Saif et al., "Nanometer level characterization of the James Webb Space Telescope optomechanical systems using high-speed interferometry," Appl. Opt. 54(13), 4285-4298 (2015).

41. P. Yoder and D. Vukobratovich, Opto-Mechanical Systems Design, Two Volume Set, 4th ed., CRC Press, Boca Raton, Florida (2015).

42. H. D. Hall, "Problems in adapting small mirror fabrication techniques to large mirrors," in Opt. Telesc. Technol. (A Workshop), NASA-SP-233, NASA Headquarters, Washington, D.C., pp. 149-152 (1970). https://ntrs.nasa.gov/search.jsp?R=19700027360.

43. L. Montagnino et al., "Test and evaluation of a 60-inch test mirror," Proc. SPIE 0183 (1979).

44. M. H. Krim, "Metrology mount development and verification for a large spaceborne mirror," Proc. SPIE 0332, 440-445 (1982).

45. L. A. Montagnino, "Test and evaluation of the Hubble Space Telescope 2.4-meter primary mirror," Proc. SPIE 0571, 182 (1986).

46. J. W. Zinn and G. W. Jones, "Kepler primary mirror assembly: FEA surface figure analyses and comparison to metrology," Proc. SPIE 6671, 667105 (2007).

47. C. J. Evans and R. N. Kestner, "Test optics error removal," Appl. Opt. 35(7), 1015-1021 (1996).

48. M. Zolcinski-Couet, J. A. Magner, and David A. Zweig, "Absolute calibration of flats for densely sampled data," Proc. SPIE 2536, 1-8 (1995).

49. R. Egerman et al., "Status of the Advanced Mirror Technology Development (AMTD) phase 2 1.5m ULE mirror," Proc. SPIE 9575, 95750L (2015).

50. W. H. Arnold and H. P. Stahl, "Influence of core and hexapod geometry, and local reinforcement on the performance of ultra lightweight ULE mirror," Proc. SPIE 10743, 107430B (2018).

51. W. R. Arnold and H. P. Stahl, "Design trade study for a 4-meter off-axis primary mirror substrate and mount for the Habitable-zone Exoplanet Direct Imaging Mission," Proc. SPIE 10398, 1039808 (2017).

52. J. M. Davis et al., "HabEx primary mirror trade studies," Proc. SPIE 10371, 103710B (2017).

53. W. R. Arnold, R. M. Bevan, and H. P. Stahl, "Integration of mirror design with suspension system using NASA's new mirror modeling software," Proc. SPIE 8836, 88360J (2013).

54. W. R. Arnold, "Evolving design criteria for very large aperture space-based telescopes and their influence on the need for integrated tools in the optimization process," Proc. SPIE 9573, 95730G (2015).

55. T. E. Brooks et al., "Optothermal stability of large ULE and Zerodur mirrors," Proc. SPIE 10743, 107430A (2018).

56. G. W. Matthews et al., "The development of stacked core technology for the fabrication of deep lightweight UV-quality space mirrors," Proc. SPIE 8838, 88380L (2013).

57. T. Hull et al., "Practical aspects of specification of extreme lightweight ZERODUR mirrors for spaceborne missions," Proc. SPIE 8836, 883607 (2013).

58. P. Hartmann, "Minimum lifetime of ZERODUR ${ }^{\circledR}$ structures based on the breakage stress threshold model: a review," Opt. Eng. 58(2), 020902 (2019).

59. T. S. Chonis et al., "Characterization and calibration of the James Webb space telescope mirror actuators fine stage motion," Proc. SPIE 10698, 106983S (2018).

60. L3Harris, "SpaceviewTM 50/65/70 small satellite imaging solutions," Specification Data Sheet, Harris Corporation, 2019, https://www.harris.com/.

61. L3Harris, "SpaceviewTM 110 satellite imaging solution," Specification Data Sheet, Harris Corporation, 2019. https://www.harris.com/.

62. Harris Corporation, "PTC thermal system design review," NASA Marshall Space Flight Center Contract NNM15AA01C, AMTD II (an internal document) (2018).

63. T. Brooks, H. P. Stahl, and W. R. Arnold, "Advanced Mirror Technology Development (AMTD) thermal trade studies," Proc. SPIE 9577, 957703 (2015). 
64. T. E. Brooks, "Predictive thermal control applied to HabEx," Proc. SPIE 10398, 1039814 (2017).

65. K. Havey, Harris Corporation, private communication (2019).

66. Lake Shore Cryotonics, Inc., "PT-100 series platinum RTDs," https://www.lakeshore.com/ docs/default-source/product-downloads/lstc_platinum_l.pdf?sfvrsn=9157869c_5 (2019).

67. T. Westerhoff, Schott Corporation, private communication (2019).

68. J. Ziemer et al., "Colloid micro-Newton thrusters for precision attitude control," CL\#172067 (2017).

69. J. Nissin, JPL Thermal Engineer, private communication (2017).

70. A. Kissil, "SFE correction for HabEx primary mirror 4m monolithic/lightweighted ULE status update," an internal analysis performed for HabEx (2018).

71. J. B. Knight, H. P. Stahl, and T. Brooks, "Dynamic analyses of the proposed habitable exoplanet astrophysics facility," Proc. SPIE 11103, 111030F (2019).

H. Philip Stahl is a senior optical physicist at NASA Marshall Space Flight Center currently leading the telescope design study for the potential Habitable Exoplanet Observer mission and is principle investigator developing advanced technology to manufacture, test, and thermally control large mirror systems. Previous NASA assignments include principle investigator for the Advanced Mirror Technology Development project; mirror technology lead for the James Webb Space Telescope, including development of JWST mirror fabrication and testing technology and providing insight/oversight of JWST optical components; and various technology roadmap studies. Additionally, he was responsible for fabrication and test of the Spitzer secondary mirror, alignment, and operation of the Air Force 4-meter 7-segment LAMP mirror, absolute calibration of an LIGO test flat, testing of the Keck primary mirror segments, and designing human-operated optical systems for Shuttle microgravity experiments. He is a leading authority in optical metrology, optical engineering, and phase-measuring interferometry. Many of the world's largest telescopes have been fabricated with the aid of high-speed and infrared phasemeasuring Interferometers developed by him, including the Keck, VLT, and Gemini telescopes. He is a fellow of SPIE, fellow of OSA, and member of AAS and IAU. He was the 2014 SPIE President and an ICO Vice-President, in 2005 to 2011. He received his PhD in 1985 and his MS degree in 1983 in optical science at the University of Arizona Optical Sciences Center. He received his BA degree in physics and mathematics from Wittenberg University in 1979.

Gary Kuan is a senior optical engineer at the NASA Jet Propulsion Laboratory/California Institute of Technology, where he has worked since 2000. He is the design lead for the Habitable Exoplanet Observatory (HabEx) decadal study and the optical system engineer for the WFIRST Coronagraph Instrument. Previously, he was the deputy manager of the WFIRST telescope at JPL. He received his PhD in 2001 in electrical engineering: applied physics from the University of California, San Diego, and his BS degree in 1992 in electrical engineering from the University of California, Los Angeles.

William R. Arnold is a subject matter expert in optical-mechanics at Marshall Space Flight Center, where he is currently developing large lightweight mirror design software. He has $50+$ years of engineering experience with expertise in structural dynamic, shock and vibration, and fracture mechanics. He designed the primary mirror and suspension system for the Kepler Space Telescope, the turret optics for airborne laser, and numerous large ground-based telescopes.

Thomas Brooks is an aerospace engineer at Marshall Space Flight Center. He is coinvestigator on Predictive Thermal Control (a Strategic Astrophysics Technology grant from NASA HQ). He has a background in numerical modeling with a focus in thermal and structural analysis, and he writes machine learning and artificial intelligence code in Python to perform optimization and model correlation, as well as to write control system algorithms. He has bachelor's and master's degrees in aerospace engineering.

J. Brent Knight is a senior dynamics analyst at NASA Marshall Space Flight Center. Experience includes stability analyses for ISS microgravity and space optical systems, dynamic 
structural design load development, and development of new methods to mitigate unnecessary mass in structural designs. Projects supported include ISS, SPACEHAB, Ares, SLS, HabEx, Lynx, IXPE, EUSO, and others. He received his BS and MS degrees in mechanical engineering, Mississippi State University, 1986/1988, as well as an MS degree in bioengineering, Clemson University, 1999.

Stefan Martin is a senior optical engineer at the Jet Propulsion Laboratory. He received his BSc degree in physics from the University of Bristol, United Kingdom, and his $\mathrm{PhD}$ in engineering from the University of Wales. At JPL, he has been leader of the TPF-I Flight Instrument Engineering Team, testbed lead for the TPFI Planet Detection Testbed, and payload lead for the HabEx telescope design study. He is currently involved in starshade accommodation on future space telescopes, such as WFIRST. 